\title{
Part III Result of Treatments
}

\section{COMPUTATION OF SURVIVAL RATE}

Siduey J. Cutler

LX …….. The number alive at the beginning of interval

DX ….... The number died during the interval

UX ……. The number lost to follow-up during the interval

WX ……. The number withdrawn alive during the interval

$\mathrm{L}^{\prime} \mathrm{X}=\mathrm{LX}-1 / 2(\mathrm{UX}+\mathrm{WX})$

$\mathrm{QX}=\mathrm{DX} / \mathrm{L}^{\prime} \mathrm{X}$

$\mathrm{PX}=1-\mathrm{QX}$

$\mathrm{P}^{\prime} \mathrm{X}=\mathrm{P}_{1} \times \mathrm{P}_{2} \times \cdots($ Cumulative proportion surviving $)$

Q: The survival probability of each individual patient was obtained for annual interval of follow-up, taking into account his(her) age at the beginning of the interval.

* S.J.Cutler: Computation of srvival rate NCL Monograph 15: 381-385, 1964

Cases died within 30 days from the beginning of treatment were excluded for calculation of survival rate.

\section{DEFINITION OF VARIOUS CLASSIFICATIONS OF GLIOMAS BY CODE NUMBER\#}

All gliomas ( code of histology 1-18)

High grade gliomas ( code of histology 2, 4, 5, 6, 9, 11, 14, 16, 17, 18 )

Glioblastma ( code of histology 17 )

Low grade gliomas ( code of histology 1, 3, 7, 8, 10, 13, 15 )

Medulloblastoma ( code of histology 18 )

Pontine gliomas ( code of sites 20; code of histology 8, 9, 17, 49 )

\# See classification of tumor sites or histology ( pages vi-vii ).

MANTEL'S CHI-SQUARE TEST:

EVALUATION OF DIFFERENCE IN WHOLE SURVIVAL TIME PATTERNS

$* \quad=5 \%$ significant $\quad\left(\chi^{2}>3.84\right)$

$* * \quad=1 \%$ significant $\left(\chi^{2}>6.63\right)$

$* * *=0.1 \%$ significant $\left(\chi^{2}>10.24\right)$ 


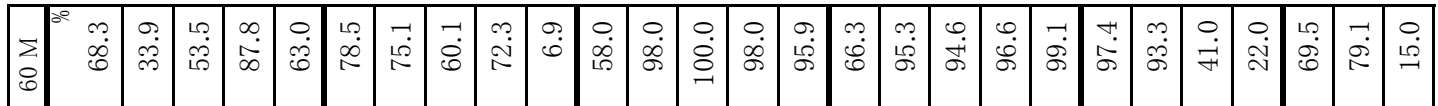

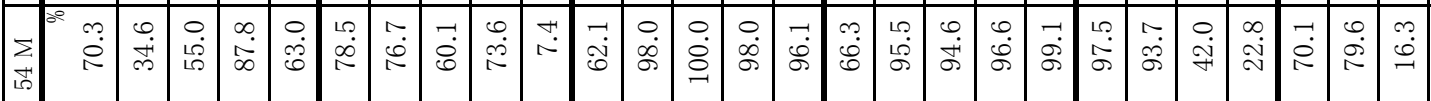

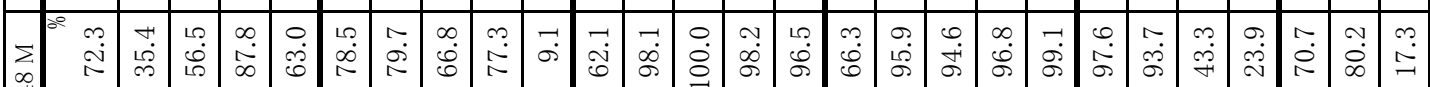

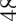

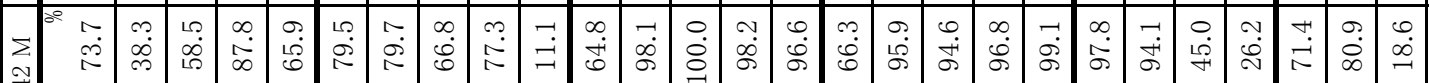

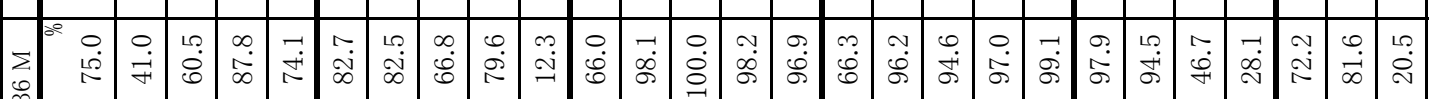

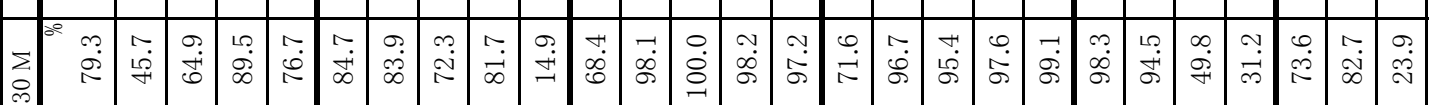

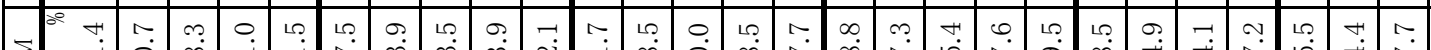
$\sum_{\sim}$

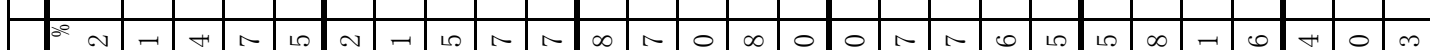

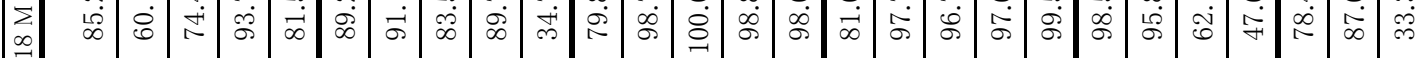

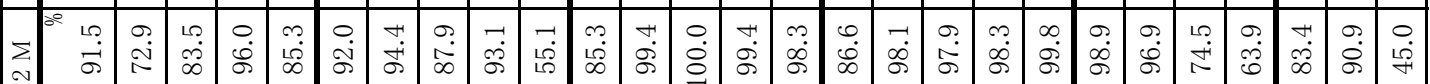

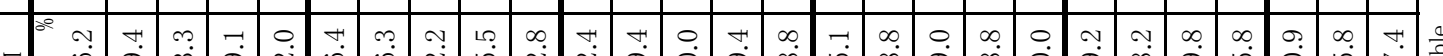

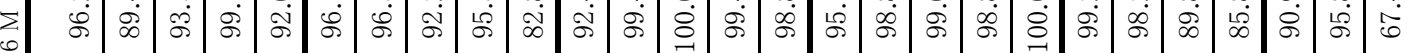
*

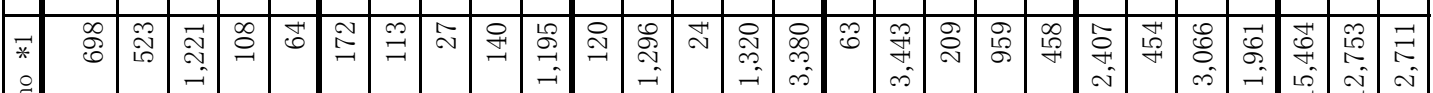
(1) - 


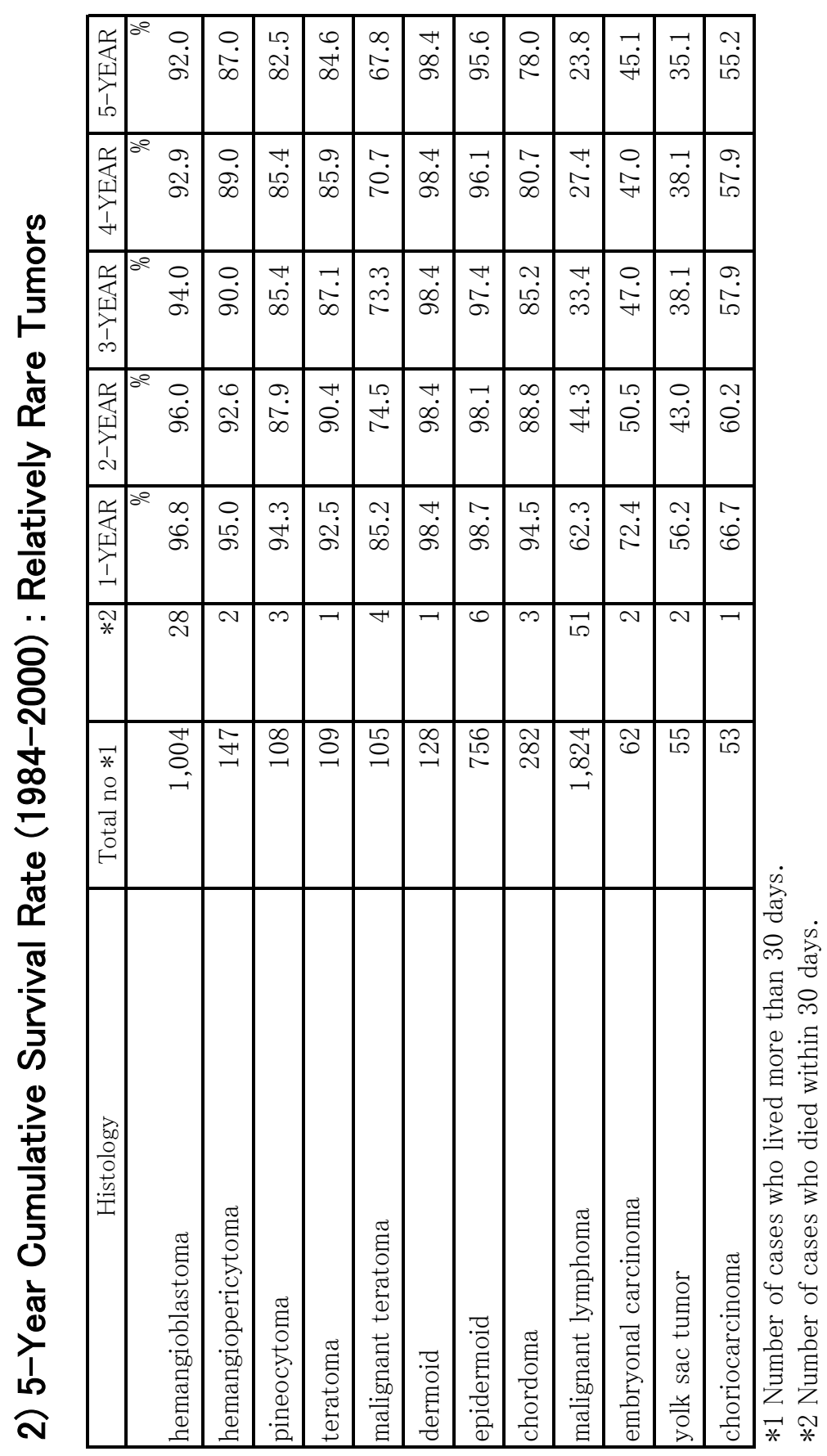




\section{Survival Rates of Primary Brain Tumors Classified by Operation, Radiation, Clinical Gradings (1984-2000)}

1) Meningioma

Operation (1984-2000): Cumulative Survival Rate

\begin{tabular}{|ll|r|r|r|r|r|r|r|r|}
\hline & PATTERN & \multicolumn{2}{|c|}{ NO. OF CASES $* 1$} & $* 2$ & 1-YEAR & 2-YEAR & 3-YEAR & 4-YEAR & 5-YEAR \\
\hline PAT 0 & none & 892 & 5.7 & 30 & 95.5 & 92.6 & 88.4 & 86.0 & 84.2 \\
PAT 1 decompression & 83 & 0.5 & 5 & 94.9 & 87.8 & 87.8 & 87.8 & 85.8 \\
PAT 2 biopsy or partial & 350 & 2.2 & 9 & 91.2 & 88.1 & 85.8 & 84.1 & 82.7 \\
PAT 3 50\% resected & 436 & 2.8 & 4 & 94.7 & 92.0 & 89.3 & 87.7 & 85.9 \\
PAT 4 75\% resected & 1,142 & 7.2 & 22 & 95.7 & 93.7 & 92.2 & 90.1 & 88.7 \\
PAT 5 95\% resected & 2,930 & 18.6 & 37 & 97.9 & 96.8 & 95.8 & 95.0 & 94.2 \\
PAT 6 total removal & 9,948 & 63.0 & 87 & 98.1 & 97.3 & 96.5 & 95.9 & 95.0 \\
\hline \multicolumn{1}{c}{ total } & 15,781 & 100.0 & 194 & & & & & \\
\hline
\end{tabular}

$* 1$ Number of cases who lived more than 30 days.

*2 Number of cases who died within 30 days.

Mantel Chi-Square Test

\begin{tabular}{|c|c|c|c|c|c|c|c|}
\hline & DAT 0 & PAT 1 & PAT 2 & PAT 3 & PAT 4 & PAT 5 & PAT 6 \\
\hline PAT 0 & -1.00 & 0.00 & 1.13 & 0.23 & $\begin{array}{r}* \\
5.14\end{array}$ & $\begin{array}{r}\text { **** } \\
60.21\end{array}$ & $\begin{array}{r}\text { **** } \\
111.26\end{array}$ \\
\hline PAT 1 & 0.00 & -1.00 & 0.34 & 0.02 & 0.80 & $\begin{array}{r}* * * \\
9.43\end{array}$ & $\begin{array}{r}\text { **** } \\
13.58\end{array}$ \\
\hline PAT 2 & 1.13 & 0.34 & -1.00 & 1.60 & $\begin{array}{r}\text { *** } \\
8.37\end{array}$ & $\begin{array}{r}* * * \\
56.01\end{array}$ & $\begin{array}{r}\text { *** } \\
89.33\end{array}$ \\
\hline PAT 3 & 0.23 & 0.02 & 1.60 & -1.00 & 1.97 & $\begin{array}{r}\text { **** } \\
33.21\end{array}$ & $\begin{array}{r}* * * \\
56.07\end{array}$ \\
\hline PAT 4 & $\begin{array}{r}* * \\
5.14\end{array}$ & 0.80 & $\begin{array}{r}* * \\
8.37\end{array}$ & 1.97 & -1.00 & $\begin{array}{r}* * * \\
27.87\end{array}$ & $\begin{array}{r}\text { **** } \\
58.97\end{array}$ \\
\hline PAT 5 & $\begin{array}{r}* * * \\
60.21\end{array}$ & $\begin{array}{r}* * \\
9.43\end{array}$ & $\begin{array}{r}* * * * \\
56.01\end{array}$ & $\begin{array}{r}* * * \\
33.21\end{array}$ & $\begin{array}{r}\text { *** } \\
27.87\end{array}$ & -1.00 & 2.33 \\
\hline PAT 6 & $\begin{array}{r}\text { *** } \\
111.26\end{array}$ & $\begin{array}{r}* * * \\
13.58 \\
\end{array}$ & $\begin{array}{r}* * * \\
89.33 \\
\end{array}$ & $\begin{array}{r}* * * \\
56.07\end{array}$ & $\begin{array}{r}* * * * \\
58.97\end{array}$ & 2.33 & -1.00 \\
\hline
\end{tabular}

\section{2) Malignant Meningioma}

Operation (1984-2000) : Cumulative Survival Rate

\begin{tabular}{|ll|r|r|r|r|r|r|r|r|}
\hline & PATTERN & \multicolumn{2}{|c|}{ NO. OF CASES $* 1$} & $* 2$ & 1-YEAR & 2-YEAR & 3-YEAR & 4-YEAR & 5-YEAR \\
\hline PAT 0 none & 10 & 3.0 & 0 & 57.9 & 57.9 & 57.9 & 57.9 & 57.9 \\
PAT 1 decompression & 0 & 0.0 & 0 & - & - & - & - & - \\
PAT 2 & biopsy or partial & 11 & 3.3 & 1 & 50.0 & 50.0 & 50.0 & 50.0 & 35.7 \\
PAT 3 50\% resected & 13 & 3.9 & 0 & 91.3 & 91.3 & 81.2 & 60.9 & 60.9 \\
PAT 4 & 75\% resected & 35 & 10.4 & 0 & 91.2 & 87.9 & 76.1 & 63.1 & 58.6 \\
PAT 5 & 95\% resected & 122 & 36.2 & 2 & 88.1 & 81.6 & 71.3 & 63.1 & 56.5 \\
PAT 6 total removal & 146 & 43.3 & 0 & 92.0 & 86.1 & 81.3 & 75.7 & 71.9 \\
\hline \multicolumn{1}{c}{ total } & 337 & 100.0 & 3 & & & & & \\
\hline
\end{tabular}

*1 Number of cases who lived more than 30 days.

$* 2$ Number of cases who died within 30 days. 
Mantel Chi-Square Test

\begin{tabular}{|l|r|r|r|r|r|r|r|}
\hline & \multicolumn{1}{|c|}{ PAT 0 } & \multicolumn{1}{c|}{ PAT 1 } & \multicolumn{1}{c|}{ PAT 2 } & \multicolumn{1}{c|}{ PAT 3 } & PAT 4 & PAT 5 & \multicolumn{1}{c|}{ PAT 6 } \\
\hline PAT 0 & -1.00 & -1.00 & 0.21 & 0.30 & 0.60 & 0.53 & 2.33 \\
\hline PAT 1 & -1.00 & -1.00 & -1.00 & -1.00 & -1.00 & -1.00 & -1.00 \\
\hline PAT 2 & 0.21 & -1.00 & -1.00 & 0.96 & 1.91 & 2.17 & 6.17 \\
\hline PAT 3 & 0.30 & -1.00 & 0.96 & -1.00 & 0.03 & 0.09 & 0.36 \\
\hline PAT 4 & 0.60 & -1.00 & 1.91 & 0.03 & -1.00 & 0.12 & 0.97 \\
\hline PAT 5 & 0.53 & -1.00 & 2.17 & 0.09 & 0.12 & -1.00 & 3.91 \\
\hline PAT 6 & 2.33 & -1.00 & 6.17 & 0.36 & 0.97 & 3.91 & -1.00 \\
\hline
\end{tabular}

\section{3) Neurinoma (Schwannoma)}

Operation (1984-2000): Cumulative Survival Rate

\begin{tabular}{|ll|r|r|r|r|r|r|r|r|}
\hline & PATTERN & \multicolumn{2}{|c|}{ NO. OF CASES $* 1$} & $* 2$ & 1-YEAR & 2-YEAR & 3-YEAR & 4-YEAR & 5-YEAR \\
\hline PAT 0 & none & 311 & 4.9 & 4 & 97.9 & 95.3 & 94.3 & 94.3 & 94.3 \\
PAT 1 & decompression & 34 & 0.5 & 0 & 90.6 & 87.0 & 87.0 & 87.0 & 81.6 \\
PAT 2 biopsy or partial & 136 & 2.2 & 1 & 99.2 & 96.5 & 91.7 & 90.6 & 90.6 \\
PAT 3 50\% resected & 221 & 3.5 & 2 & 97.1 & 96.0 & 94.8 & 92.1 & 90.6 \\
PAT 4 75\% resected & 947 & 15.1 & 15 & 98.0 & 97.4 & 96.2 & 95.1 & 94.8 \\
PAT 5 95\% resected & 1,964 & 31.2 & 12 & 98.6 & 97.8 & 97.4 & 96.7 & 96.5 \\
PAT 6 total removal & 2,675 & 42.5 & 13 & 98.9 & 98.5 & 98.2 & 97.9 & 97.5 \\
\hline \multicolumn{1}{c}{ total } & 6,288 & 100.0 & 47 & & & & & \\
\hline
\end{tabular}

$* 1$ Number of cases who lived more than 30 days.

$* 2$ Number of cases who died within 30 days.

\section{Mantel Chi-Square Test}

\begin{tabular}{|c|c|c|c|c|c|c|c|}
\hline & PAT 0 & PAT 1 & PAT 2 & PAT 3 & PAT 4 & PAT 5 & PAT 6 \\
\hline PAT 0 & -1.00 & $\begin{array}{r}* \\
5.22\end{array}$ & 0.76 & 0.95 & 0.37 & 3.53 & $\begin{array}{r}* * \\
9.81\end{array}$ \\
\hline PAT 1 & $\begin{array}{r}* * \\
5.22 \\
\end{array}$ & -1.00 & 2.16 & 2.27 & $\begin{array}{r}* * \\
8.53 \\
\end{array}$ & $\begin{array}{r}* * * \\
15.74 \\
\end{array}$ & $\begin{array}{r}* * * \\
25.44 \\
\end{array}$ \\
\hline PAT 2 & 0.76 & 2.16 & -1.00 & 0.00 & 2.55 & $\begin{array}{r}* * * \\
7.97 \\
\end{array}$ & $\begin{array}{r}* * * \\
16.10 \\
\end{array}$ \\
\hline PAT 3 & 0.95 & 2.27 & 0.00 & -1.00 & 3.57 & $\begin{array}{r}* * * \\
11.45 \\
\end{array}$ & $\begin{array}{r}\text { *** } \\
23.12 \\
\end{array}$ \\
\hline PAT 4 & 0.37 & $\begin{array}{r}* * \\
8.53 \\
\end{array}$ & 2.55 & 3.57 & -1.00 & 3.45 & $\begin{array}{r}\text { *** } \\
13.17 \\
\end{array}$ \\
\hline PAT 5 & 3.53 & $\begin{array}{r}* * * \\
15.74 \\
\end{array}$ & $\begin{array}{r}* * * \\
7.97 \\
\end{array}$ & $\begin{array}{r}* * * \\
11.45 \\
\end{array}$ & 3.45 & -1.00 & 3.67 \\
\hline PAT 6 & $\begin{array}{r}* * \\
9.81\end{array}$ & $\begin{array}{r}\text { **** } \\
25.44\end{array}$ & $\begin{array}{r}\text { *** } \\
16.10\end{array}$ & $\begin{array}{r}\text { **** } \\
23.12\end{array}$ & $\begin{array}{r}\text { *** } \\
13.17\end{array}$ & 3.67 & -1.00 \\
\hline
\end{tabular}


4) Von Recklinghausen's Disease

Operation (1984-2000): Cumulative Survival Rate

\begin{tabular}{|ll|r|r|r|r|r|r|r|r|}
\hline & PATTERN & NO. OF CASES $* 1$ & $* 2$ & 1-YEAR & 2-YEAR & 3-YEAR & 4-YEAR & 5-YEAR \\
\hline PAT 0 & none & 26 & 17.2 & 0 & 95.9 & 86.8 & 86.8 & 86.8 & 86.8 \\
PAT 1 & decompression & 3 & 2.0 & 0 & 66.7 & 66.7 & 66.7 & 66.7 & 66.7 \\
PAT 2 biopsy or partial & 14 & 9.3 & 0 & 100.0 & 91.7 & 81.5 & 81.5 & 81.5 \\
PAT 3 50\% resected & 14 & 9.3 & 0 & 100.0 & 90.9 & 90.9 & 90.9 & 79.5 \\
PAT 4 & 75\% resected & 29 & 19.2 & 0 & 100.0 & 100.0 & 100.0 & 100.0 & 92.5 \\
PAT 5 & 95\% resected & 33 & 21.9 & 0 & 100.0 & 96.6 & 93.0 & 93.0 & 89.2 \\
PAT 6 & total removal & 32 & 21.2 & 0 & 93.2 & 93.2 & 93.2 & 93.2 & 93.2 \\
\hline \multicolumn{1}{c}{ total } & 151 & 100.0 & 0 & & & & & \\
\hline
\end{tabular}

$* 1$ Number of cases who lived more than 30 days.

$* 2$ Number of cases who died within 30 days.

Mantel Chi-Square Test : No Significancy Detected between Each Two of PATs

5) Craniopharyngioma

a) Operation (1984-2000): Cumulative Survival Rate

\begin{tabular}{|c|c|c|c|c|c|c|c|c|c|}
\hline & PATTERN & NO. OF & $\mathrm{ES} * 1$ & $* 2$ & 1-YEAR & 2-YEAR & 3-YEAR & 4-YEAR & 5-YEAR \\
\hline & & & $\%$ & & & $\%$ & $\%$ & $\%$ & $\%$ \\
\hline PAT 0 & none & 96 & 4.5 & 7 & 95.4 & 91.4 & 89.9 & 88.3 & 88.3 \\
\hline PAT 1 & decompression & 84 & 3.9 & 2 & 96.4 & 93.6 & 90.4 & 86.9 & 84.9 \\
\hline PAT 2 & biopsy or partial & 356 & 16.7 & 3 & 95.9 & 92.0 & 90.2 & 89.0 & 87.3 \\
\hline PAT 3 & $50 \%$ resected & 202 & 9.5 & 2 & 93.8 & 91.5 & 90.9 & 87.5 & 85.2 \\
\hline PAT 4 & $75 \%$ resected & 423 & 19.9 & 5 & 94.3 & 91.3 & 89.5 & 88.2 & 86.9 \\
\hline PAT 5 & $95 \%$ resected & 481 & 22.6 & 3 & 96.5 & 95.1 & 94.0 & 93.4 & 92.1 \\
\hline PAT 6 & total removal & 488 & 22.9 & 7 & 95.9 & 95.5 & 95.2 & 93.8 & 92.8 \\
\hline & total & 2,130 & 100.0 & 29 & & & & & \\
\hline
\end{tabular}

*1 Number of cases who lived more than 30 days.

$* 2$ Number of cases who died within 30 days.

\section{Mantel Chi-Square Test}

\begin{tabular}{|l|r|r|r|r|r|r|r|}
\hline & PAT 0 & PAT 1 & PAT 2 & PAT 3 & PAT 4 & PAT 5 & PAT 6 \\
\hline PAT 0 & -1.00 & 0.12 & 0.01 & 0.23 & 0.08 & 1.33 & 1.85 \\
\hline PAT 1 & 0.12 & -1.00 & 0.12 & 0.00 & 0.04 & 2.85 & 3.67 \\
\hline PAT 2 & 0.01 & 0.12 & -1.00 & 0.33 & 0.06 & 4.07 & $\begin{array}{r}* \\
*\end{array}$ \\
\hline PAT 3 & 0.23 & 0.00 & 0.33 & -1.00 & 0.14 & 5.64 & 7.06 \\
\hline PAT 4 & 0.08 & 0.04 & 0.06 & 0.14 & -1.00 & 5.60 & 7.19 \\
\hline PAT 5 & 1.33 & 2.85 & 4.07 & 5.64 & 5.60 & -1.00 & 0.11 \\
\hline PAT 6 & 1.85 & 3.67 & 5.40 & 7.06 & 7.19 & 0.11 & -1.00 \\
\hline
\end{tabular}


b) Operation With or Without Radiation Therapy (1984-2000) : Cumulative Survival Rate

\begin{tabular}{|c|c|c|c|c|c|c|c|c|c|}
\hline & PATTERN & NO. OF & $\mathrm{S} * 1$ & $* 2$ & 1-YEAR & 2-YEAR & 3-YEAR & 4-YEAR & 5-YEAR \\
\hline & & & $\%$ & & $\%$ & $\%$ & $\%$ & $\%$ & $\%$ \\
\hline PAT 1 & $\begin{array}{l}\text { ope (biopsy) + } \\
\text { radiation }\end{array}$ & 123 & 6.3 & 0 & 98.3 & 95.5 & 94.5 & 93.4 & 91.1 \\
\hline PAT 2 & ope (biopsy) alone & 230 & 11.9 & 3 & 94.6 & 90.0 & 87.7 & 86.4 & 85.1 \\
\hline PAT 3 & $\begin{array}{l}\text { ope }(50-75 \% \text { resected }) \\
\text { + radiation }\end{array}$ & 266 & 13.7 & 0 & 96.9 & 94.8 & 94.0 & 92.5 & 90.5 \\
\hline PAT 4 & $\begin{array}{l}\text { ope }(50-75 \% \\
\text { resected) alone }\end{array}$ & 357 & 18.4 & 7 & 92.0 & 88.6 & 86.7 & 84.3 & 83.1 \\
\hline PAT 5 & $\begin{array}{l}\text { ope }(\geqq 95 \% \text { resected }) \\
\text { + radiation }\end{array}$ & 138 & 7.1 & 0 & 97.8 & 96.2 & 95.3 & 95.3 & 92.0 \\
\hline PAT 6 & $\begin{array}{l}\text { ope }(\geqq 95 \% \\
\text { resected }) \text { alone }\end{array}$ & 826 & 42.6 & 10 & 95.9 & 95.1 & 94.5 & 93.3 & 92.5 \\
\hline PAT 7 & $\begin{array}{l}\text { ope }(\text { all cases })+ \\
\text { radiation }\end{array}$ & 552 & 27.3 & 0 & 97.4 & 95.0 & 93.9 & 92.5 & 90.2 \\
\hline PAT 8 & $\begin{array}{l}\text { ope alone } \\
\text { (all cases) }\end{array}$ & 1471 & 72.7 & 22 & 94.8 & 92.8 & 91.5 & 90.1 & 89.0 \\
\hline
\end{tabular}

*1 Number of cases who lived more than 30 days.

$* 2$ Number of cases who died within 30 days.

Mantel Chi-Square Test

\begin{tabular}{|l|r|r|r|r|r|r|r|r|}
\hline & PAT 1 & PAT 2 & PAT 3 & PAT 4 & PAT 5 & PAT 6 & PAT 7 & PAT 8 \\
\hline PAT 1 & -1.00 & 2.54 & 0.06 & 4.29 & 0.06 & 0.09 & 0.09 & 0.66 \\
\hline PAT 2 & 2.54 & -1.00 & 3.24 & 0.38 & 3.83 & 9.30 & 4.49 & 2.27 \\
\hline PAT 3 & 0.06 & 3.24 & -1.00 & 6.44 & 0.30 & 0.65 & 0.00 & 0.65 \\
\hline PAT 4 & 4.29 & 0.38 & 6.44 & -1.00 & 6.04 & 18.85 & 9.93 & 7.50 \\
\hline PAT 5 & 0.06 & 3.83 & 0.30 & 6.04 & -1.00 & 0.00 & 0.41 & 1.44 \\
\hline PAT 6 & 0.09 & 9.30 & 0.65 & $18 * 85$ & 0.00 & -1.00 & 1.10 & 5.74 \\
\hline PAT 7 & 0.09 & 4.49 & 0.00 & 9.93 & 0.41 & 1.10 & -1.00 & 1.06 \\
\hline PAT 8 & 0.66 & 2.27 & 0.65 & 7.50 & 1.44 & 5.74 & 1.06 & -1.00 \\
\hline
\end{tabular}

6) Germinoma

Radiation Therapy (1984-2000) : Cumulative Survival Rate

\begin{tabular}{|cl|r|r|r|r|r|r|r|r|}
\hline & PATTERN & \multicolumn{2}{|c|}{ NO. OF CASES $* 1$} & $* 2$ & 1-YEAR & 2-YEAR & 3-YEAR & 4-YEAR & 5-YEAR \\
\hline \multirow{2}{*}{ PAT 0 } & none & 117 & 10.0 & 4 & 87.7 & 85.8 & 84.8 & 83.7 & 81.2 \\
PAT 1 & irradiated & 1053 & 90.0 & 2 & 96.9 & 93.9 & 93.1 & 91.9 & 91.0 \\
\hline \multicolumn{2}{|c|}{ total } & 1170 & 100.0 & 6 & & & & & \\
\hline
\end{tabular}

*1 Number of cases who lived more than 30 days.

$* 2$ Number of cases who died within 30 days. 
7) All Glioma (Histology Code 1-18)

a) Operation (1984-2000) : Cumulative Survival Rate

\begin{tabular}{|ll|r|r|r|r|r|r|r|r|}
\hline & PATTERN & \multicolumn{2}{|c|}{ NO. OF CASES $* 1$} & $* 2$ & 1-YEAR & 2-YEAR & 3-YEAR & 4-YEAR & 5-YEAR \\
\hline PAT 0 & none & 920 & 6.0 & 46 & 57.7 & 38.3 & 31.7 & 28.3 & 26.7 \\
PAT 1 & decompression & 270 & 1.8 & 21 & 67.5 & 48.2 & 37.9 & 33.4 & 31.9 \\
PAT 2 & biopsy or partial & 3,863 & 25.3 & 101 & 63.0 & 44.0 & 37.0 & 33.8 & 31.0 \\
PAT 3 & 50\% resected & 1,572 & 10.3 & 51 & 63.9 & 42.7 & 35.3 & 31.3 & 29.1 \\
PAT 4 & 75\% resected & 3,314 & 21.7 & 77 & 71.2 & 48.3 & 40.1 & 36.3 & 32.6 \\
PAT 5 & 95\% resected & 3,373 & 22.1 & 34 & 81.0 & 60.1 & 52.9 & 47.7 & 43.8 \\
PAT 6 & total removal & 1,978 & 12.9 & 20 & 90.8 & 79.2 & 73.7 & 71.2 & 69.4 \\
\hline \multicolumn{2}{c}{ total } & 15,290 & 100.0 & 350 & & & & & \\
\hline
\end{tabular}

$* 1$ Number of cases who lived more than 30 days.

$* 2$ Number of cases who died within 30 days.

\section{Mantel Chi-Square Test}

\begin{tabular}{|c|c|c|c|c|c|c|c|}
\hline & PAT 0 & PAT 1 & PAT 2 & PAT 3 & PAT 4 & PAT 5 & PAT 6 \\
\hline PAT 0 & -1.00 & 2.40 & $\begin{array}{r}* \\
4.45\end{array}$ & 2.03 & $\begin{array}{r}* * * \\
15.99\end{array}$ & $\begin{array}{r}* * * \\
91.70\end{array}$ & $\begin{array}{r}* * * \\
351.63\end{array}$ \\
\hline PAT 1 & 2.40 & -1.00 & 0.22 & 0.64 & 0.22 & $\begin{array}{r}* * * * \\
14.50\end{array}$ & $\begin{array}{r}* * * \\
124.68 \\
\end{array}$ \\
\hline PAT 2 & $\begin{array}{r}* \\
4.45 \\
\end{array}$ & 0.22 & -1.00 & 0.50 & $\begin{array}{r}* \\
6.29 \\
\end{array}$ & $\begin{array}{r}* * * * \\
111.84 \\
\end{array}$ & $\begin{array}{r}* * * \\
472.37 \\
\end{array}$ \\
\hline PAT 3 & 2.03 & 0.64 & 0.50 & -1.00 & $\begin{array}{r}* * \\
7.57 \\
\end{array}$ & $\begin{array}{r}* * * \\
87.75 \\
\end{array}$ & $\begin{array}{r}\text { *** } \\
395.22 \\
\end{array}$ \\
\hline PAT 4 & $\begin{array}{r}* * * * \\
15.99\end{array}$ & 0.22 & $\begin{array}{r}* \\
6.29\end{array}$ & \begin{tabular}{r|}
$* *$ \\
7.57
\end{tabular} & -1.00 & $\begin{array}{r}* * * \\
65.00 \\
\end{array}$ & $\begin{array}{r}* * * \\
405.54\end{array}$ \\
\hline PAT 5 & $\begin{array}{r}* * * \\
91.70 \\
\end{array}$ & $\begin{array}{r}* * * * \\
14.50 \\
\end{array}$ & $\begin{array}{r}* * * \\
111.84 \\
\end{array}$ & $\begin{array}{r}* * * * \\
87.75 \\
\end{array}$ & $\begin{array}{r}* * * \\
65.00 \\
\end{array}$ & -1.00 & $\begin{array}{r}* * * \\
198.91 \\
\end{array}$ \\
\hline PAT 6 & $\begin{array}{r}* * * * \\
351.63\end{array}$ & $\begin{array}{r}* * * \\
124.68\end{array}$ & $\begin{array}{r}* * * * \\
472.37\end{array}$ & $\begin{array}{r}\text { *** } \\
395.22\end{array}$ & $\begin{array}{r}\text { *** } \\
405.54\end{array}$ & $\begin{array}{r}\text { *** } \\
198.91\end{array}$ & -1.00 \\
\hline
\end{tabular}

b) Radiation Therapy (1984-2000) : Cumulative Survival Rate

\begin{tabular}{|c|r|r|r|r|r|r|r|r|}
\hline PATTERN & \multicolumn{2}{|c|}{ NO. OF CASES $* 1$} & $* 2$ & 1-YEAR & 2-YEAR & 3-YEAR & 4-YEAR & 5-YEAR \\
\hline PAT 0 none & 3,548 & 22.9 & 303 & 73.8 & 66.6 & 63.4 & 61.7 & 60.7 \\
PAT 1 irradiated & 11,925 & 77.1 & 51 & 71.8 & 48.7 & 40.3 & 35.9 & 32.4 \\
\hline total & 15,473 & 100.0 & 354 & & & & & \\
\hline
\end{tabular}

*1 Number of cases who lived more than 30 days.

$* 2$ Number of cases who died within 30 days.

Mantel Chi-Square Test : Significant by $p<0.001$ 
c) Clinical Grading (1984-2000): Cumulative Survival Rate

\begin{tabular}{|c|c|c|c|c|c|c|c|c|c|}
\hline & PATTERN & NO. OF C & $\mathrm{ES} * 1$ & $* 2$ & 1-YEAR & 2-YEAR & 3-YEAR & 4-YEAR & 5-YEAR \\
\hline & & & $\%$ & & $\%$ & $\%$ & $\%$ & $\%$ & $\%$ \\
\hline PAT 1 & no symptoms & 561 & 3.6 & 5 & 92.0 & 85.2 & 81.7 & 77.7 & 74.9 \\
\hline PAT 2 & complaints only & 1,713 & 11.1 & 17 & 86.0 & 72.9 & 66.1 & 62.3 & 57.2 \\
\hline PAT 3 & focal signs & 8,328 & 54.2 & 110 & 71.6 & 50.2 & 42.3 & 38.5 & 35.8 \\
\hline PAT 4 & increased ICP & 3,299 & 21.5 & 72 & 72.4 & 52.3 & 45.4 & 41.2 & 38.2 \\
\hline PAT 5 & $\begin{array}{l}\text { disturbance of } \\
\text { consciousness }\end{array}$ & 1,302 & 8.5 & 100 & 50.6 & 29.6 & 23.5 & 21.4 & 19.6 \\
\hline PAT 6 & coma & 142 & 0.9 & 34 & 52.5 & 37.9 & 32.8 & 26.4 & 25.2 \\
\hline PAT 7 & $\begin{array}{l}\text { respiratory } \\
\text { impairment }\end{array}$ & 28 & 0.2 & 10 & 63.6 & 32.8 & 28.7 & 28.7 & 28.7 \\
\hline & total & 15,373 & 100.0 & 348 & & & & & \\
\hline
\end{tabular}

*1 Number of cases who lived more than 30 days.

$* 2$ Number of cases who died within 30 days.

\section{Mantel Chi-Square Test}

\begin{tabular}{|c|c|c|c|c|c|c|c|}
\hline & PAT 1 & PAT 2 & PAT 3 & PAT 4 & PAT 5 & PAT 6 & PAT 7 \\
\hline \multirow{2}{*}{ PAT 1} & & *** & *** & *** & *** & *** & *** \\
\hline & -1.00 & 36.69 & 171.82 & 147.59 & 273.79 & 104.50 & 31.32 \\
\hline \multirow{2}{*}{ PAT 2} & *** & & *** & *** & *** & *** & $* * *$ \\
\hline & 36.69 & -1.00 & 168.53 & 116.30 & 317.15 & 56.76 & 10.65 \\
\hline \multirow{2}{*}{ PAT 3} & *** & $* * *$ & & & *** & $* *$ & \\
\hline & 171.82 & 168.53 & -1.00 & 3.00 & 109.87 & 7.31 & 0.66 \\
\hline \multirow{2}{*}{ PAT 4} & *** & *** & & & *** & $* *$ & \\
\hline & 147.59 & 116.30 & 3.00 & -1.00 & 106.03 & 9.16 & 1.01 \\
\hline \multirow{2}{*}{ PAT 5} & *** & *** & $* * *$ & **** & & & \\
\hline & 273.79 & 317.15 & 109.87 & 106.03 & -1.00 & 0.75 & 0.62 \\
\hline \multirow{2}{*}{ PAT 6} & *** & *** & ** & ** & & & \\
\hline & 104.50 & 56.76 & 7.31 & 9.16 & 0.75 & -1.00 & 0.08 \\
\hline \multirow{2}{*}{ PAT 7} & *** & $* * *$ & & & & & \\
\hline & 31.32 & 10.65 & 0.66 & 1.01 & 0.62 & 0.08 & -1.00 \\
\hline
\end{tabular}

\section{d) Extension (1984-2000): Cumulative Survival Rate}

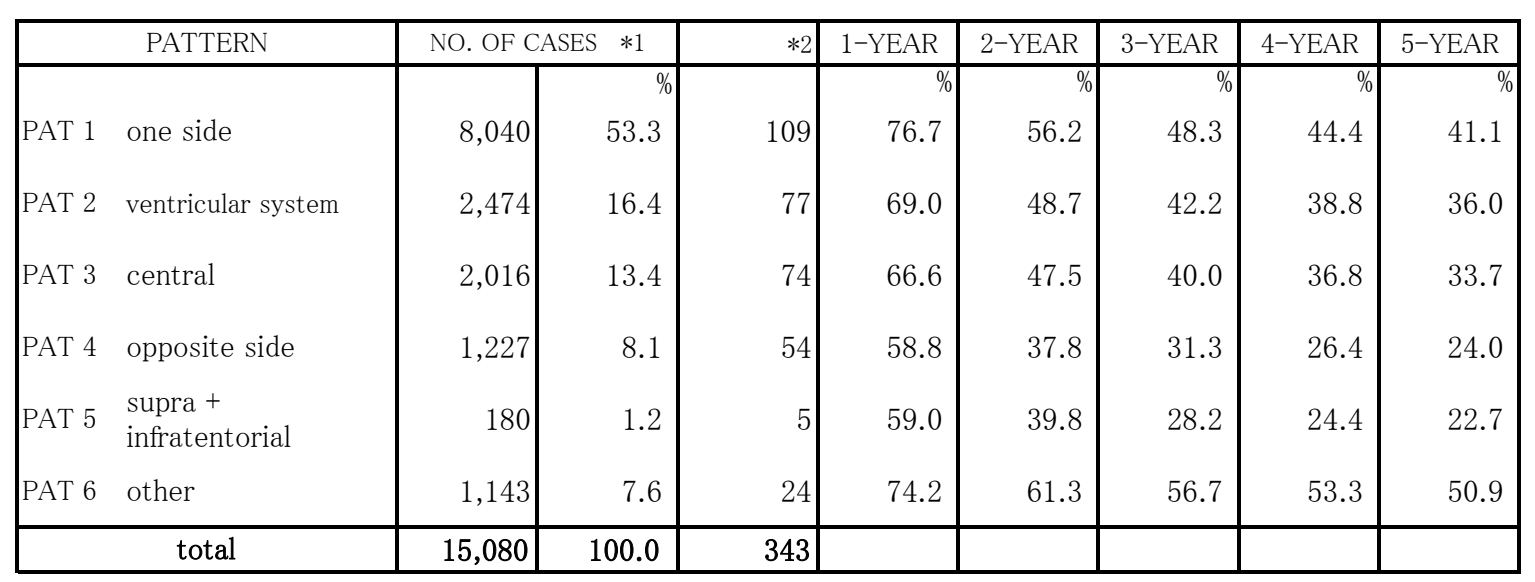

$* 1$ Number of cases who lived more than 30 days.

$* 2$ Number of cases who died within 30 days. 
Mantel Chi-Square Test

\begin{tabular}{|l|r|r|r|r|r|r|}
\hline & PAT 1 & PAT 2 & PAT 3 & PAT 4 & PAT 5 & PAT 6 \\
\hline \multirow{2}{*}{ PAT 1 } & -1.00 & 21.53 & 34.06 & 103.35 & 20.22 & $* * *$ \\
& $* * *$ & & & $* * *$ & $* *$ & 13.28 \\
\hline \multirow{2}{*}{ PAT 2 } & 21.53 & -1.00 & 1.47 & 29.13 & 7.49 & 35.27 \\
\hline \multirow{2}{*}{ PAT 3 } & $* * *$ & & & $* * *$ & $*$ & $* * *$ \\
& 34.06 & 1.47 & -1.00 & 17.61 & 4.86 & 44.98 \\
\hline \multirow{2}{*}{ PAT 4 } & $* * *$ & $* * *$ & $* * *$ & & & $* * *$ \\
& 103.35 & 29.13 & 17.61 & -1.00 & 0.04 & 91.04 \\
\hline \multirow{2}{*}{ PAT 5 } & $* * *$ & $* *$ & $*$ & & & $* * *$ \\
& 20.22 & 7.49 & 4.86 & 0.04 & -1.00 & 29.65 \\
\hline \multirow{2}{*}{ PAT 6 } & $* * *$ & $* * *$ & $* * *$ & $* * *$ & $* * *$ & \\
& 13.28 & 35.27 & 44.98 & 91.04 & 29.65 & -1.00 \\
\hline
\end{tabular}


8) High Grade Glioma (Histology Code 2,4,5,6,9,11,14,16,17,18)

a) Location (1984-2000): Cumulative Survival Rate

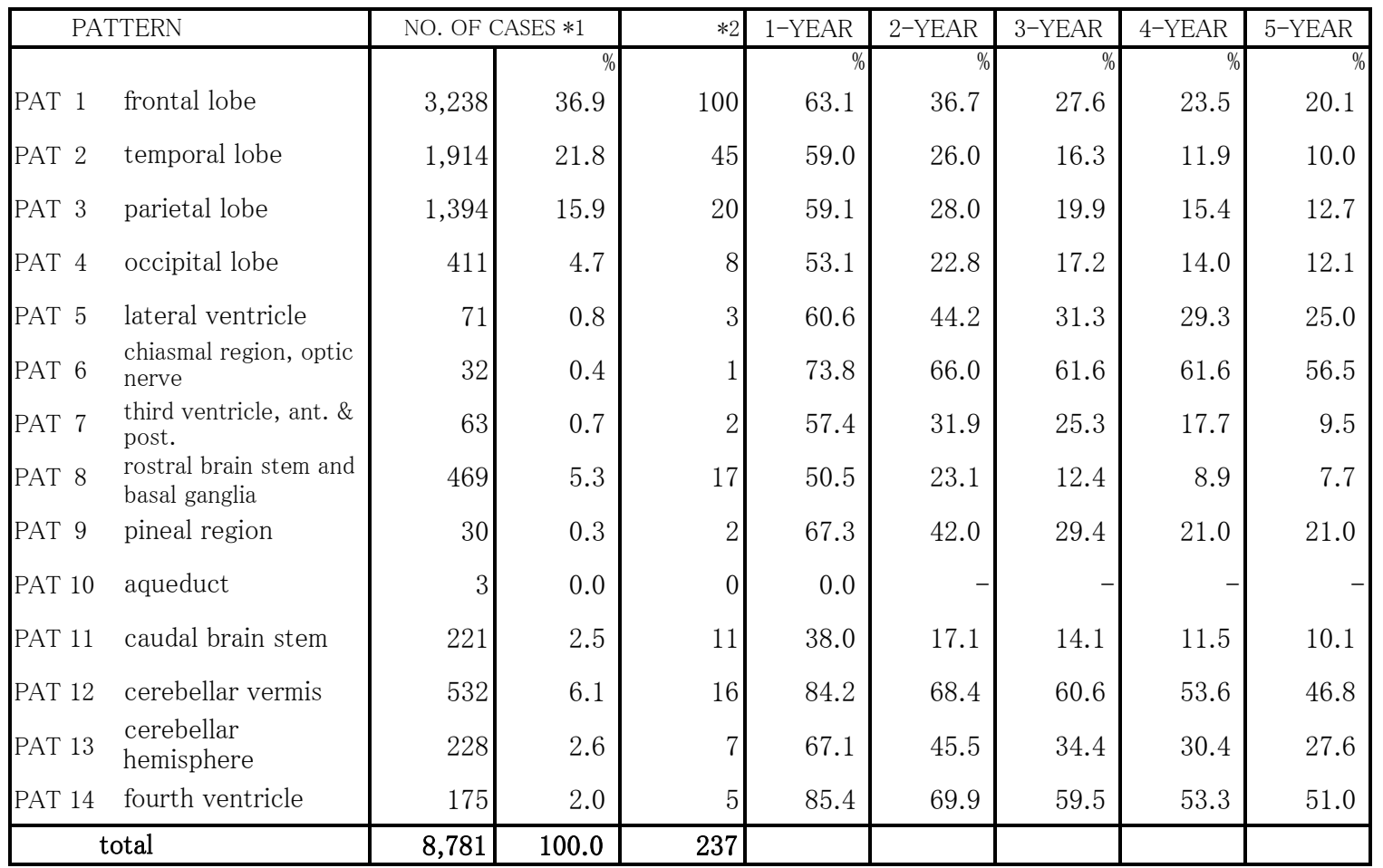

$* 1$ Number of cases who lived more than 30 days.

$* 2$ Number of cases who died within 30 days.

Mantel Chi-Square Test

\begin{tabular}{|c|c|c|c|c|c|c|c|c|c|c|c|c|c|c|}
\hline & PAT 1 & PAT 2 & PAT 3 & PAT 4 & PAT 5 & PAT 6 & PAT 7 & PAT 8 & PAT 9 & PAT 10 & PAT 11 & PAT 12 & PAT 13 & PAT 14 \\
\hline \multirow{2}{*}{ PAT 1} & & $* * *$ & $* * *$ & $* * *$ & & $* *$ & & $* * *$ & \multirow[b]{2}{*}{0.03} & \multirow[b]{2}{*}{1.62} & $* * *$ & $* * *$ & \multirow[b]{2}{*}{2.81} & \multirow{2}{*}{$\begin{array}{r}\text { *** } \\
41.25\end{array}$} \\
\hline & -1.00 & 28.76 & 13.71 & 10.75 & 0.22 & 7.07 & 1.02 & 22.40 & & & 17.77 & 96.15 & & \\
\hline \multirow{2}{*}{ PAT 2} & $* * *$ & & & & & *** & & & \multirow[b]{2}{*}{1.23} & \multirow[b]{2}{*}{1.27} & \multirow{2}{*}{$\begin{array}{r}* \\
4.88\end{array}$} & *** & \multirow{2}{*}{$\begin{array}{r}* * * \\
15.48\end{array}$} & \multirow{2}{*}{$\begin{array}{r}* * \\
73.42 \\
\end{array}$} \\
\hline & 28.76 & -1.00 & 1.04 & 0.19 & 3.21 & 12.32 & 0.06 & 2.80 & & & & 168.75 & & \\
\hline \multirow{2}{*}{ PAT 3} & $* * *$ & & & & & $* * *$ & & $*$ & \multirow[b]{2}{*}{0.72} & \multirow[b]{2}{*}{1.27} & \multirow{2}{*}{$\begin{array}{r}* \\
6.27\end{array}$} & $* * *$ & \multirow{2}{*}{$\begin{array}{r}* * * \\
10.70\end{array}$} & \multirow{2}{*}{$\begin{array}{r}\text { *** } \\
60.93\end{array}$} \\
\hline & 13.71 & 1.04 & -1.00 & 0.99 & 2.04 & 10.63 & 0.00 & 5.04 & & & & 135.37 & & \\
\hline \multirow{2}{*}{ PAT 4} & *** & & & & & $* * *$ & & & \multirow[b]{2}{*}{1.03} & \multirow[b]{2}{*}{0.89} & \multirow[b]{2}{*}{1.85} & *** & \multirow{2}{*}{$\begin{array}{r}\text { **** } \\
11.13\end{array}$} & \multirow{2}{*}{$\begin{array}{r}\text { *** } \\
53.15\end{array}$} \\
\hline & 10.75 & 0.19 & 0.99 & -1.00 & 2.74 & 10.66 & 0.11 & 0.75 & & & & 99.59 & & \\
\hline \multirow{2}{*}{ PAT 5} & & & & & & & & $*$ & \multirow[b]{2}{*}{0.02} & \multirow[b]{2}{*}{1.27} & $*$ & *** & \multirow[b]{2}{*}{0.16} & \multirow{2}{*}{$\begin{array}{r}\text { **** } \\
11.77\end{array}$} \\
\hline & 0.22 & 3.21 & 2.04 & 2.74 & -1.00 & 4.00 & 1.23 & 5.32 & & & 4.77 & 13.52 & & \\
\hline $\mathrm{PAT}$ & $* *$ & $* * *$ & $* * *$ & $* * *$ & $*$ & & $* *$ & $* * *$ & & & $* * *$ & & $*$ & \\
\hline PA1 0 & 7.07 & 12.32 & 10.63 & 10.66 & 4.00 & -1.00 & 8.18 & 14.08 & 3.47 & 2.33 & 11.96 & 0.11 & 3.90 & 0.00 \\
\hline PAT 7 & & & & & & ** & & & & & & $* * *$ & & $* * *$ \\
\hline ГА⿵ & 1.02 & 0.06 & 0.00 & 0.11 & 1.23 & 8.18 & -1.00 & 0.77 & 0.56 & 1.05 & 0.97 & 27.08 & 3.03 & 23.11 \\
\hline PAT 8 & *** & & $*$ & & & $* * *$ & & & & & & *** & *** & $* * *$ \\
\hline PAT 9 & 0.03 & 1.23 & 0.72 & 1.03 & 0.02 & 3.47 & 0.56 & 2.20 & -1.00 & 1.59 & 1.88 & $\begin{array}{r}* * \\
7.14\end{array}$ & 0.17 & $\begin{array}{r}\text { ** } \\
7.19\end{array}$ \\
\hline $\mathrm{PAT} 10$ & & & & & & & & & & & & $*$ & & $* *$ \\
\hline PA1 10 & 1.62 & 1.27 & 1.27 & 0.89 & 1.27 & 2.33 & 1.05 & 0.76 & 1.59 & -1.00 & 0.34 & 6.50 & 1.98 & 6.73 \\
\hline $\mathrm{PAT} 11$ & *** & & $*$ & & $*$ & $* * *$ & & & & & & $* * *$ & *** & *** \\
\hline ГАा 11 & 17.77 & 4.88 & 6.27 & 1.85 & 4.77 & 11.96 & 0.97 & 0.55 & 1.88 & 0.34 & -1.00 & 92.31 & 15.24 & 52.89 \\
\hline & *** & $* * *$ & $* * *$ & $* * *$ & $* * *$ & & $* * *$ & $* * *$ & $* *$ & $*$ & $* * *$ & & *** & \\
\hline PAI 12 & 96.15 & 168.75 & 135.37 & 99.59 & 13.52 & 0.11 & 27.08 & 132.57 & 7.14 & 6.50 & 92.31 & -1.00 & 23.44 & 0.32 \\
\hline$P_{2}+2$ & & *** & *** & *** & & $*$ & & *** & & & $* * *$ & $* * *$ & & $* * *$ \\
\hline ГАI 15 & 2.81 & 15.48 & 10.70 & 11.13 & 0.16 & 3.90 & 3.03 & 18.23 & 0.17 & 1.98 & 15.24 & 23.44 & -1.00 & 16.25 \\
\hline 14 & *** & *** & $* * *$ & *** & *** & & *** & *** & ** & $* *$ & *** & & *** & \\
\hline ГАІ 14 & 41.25 & 73.42 & 60.93 & 53.15 & 11.77 & 0.00 & 23.11 & 69.03 & 7.19 & 6.73 & 52.89 & 0.32 & 16.25 & -1.00 \\
\hline
\end{tabular}


b) Age-Dependent Cumulative Survival Rate (1984-2000)

\begin{tabular}{|rc|r|r|r|r|r|r|r|r|}
\hline & PATTERN & \multicolumn{2}{|c|}{ NO. OF CASES $* 1$} & $* 2$ & 1-YEAR & 2-YEAR & 3-YEAR & 4-YEAR & 5-YEAR \\
\hline PAT 1 & $0-14$ & 1,093 & 11.8 & 39 & 71.0 & 53.7 & 46.5 & 42.0 & $\%$ \\
PAT 2 & $15-50$ & 2,849 & 30.6 & 54 & 75.5 & 49.7 & 38.6 & 32.4 & 28.4 \\
PAT 3 & $51-98$ & 5,358 & 57.6 & 173 & 50.7 & 20.6 & 12.6 & 9.4 & 7.3 \\
\hline \multicolumn{2}{c|}{ total } & 9,300 & 100.0 & 266 & & & & & \\
\hline
\end{tabular}

*1 Number of cases who lived more than 30 days.

$* 2$ Number of cases who died within 30 days.

\section{Mantel Chi-Square Test}

\begin{tabular}{|l|r|r|r|}
\hline & PAT 1 & PAT 2 & PAT 3 \\
\hline PAT 1 & -1.00 & 8.55 & *** \\
& $* *$ & & 234.92 \\
\hline \multirow{2}{*}{ PAT 2 } & 8.55 & -1.00 & 342.65 \\
\hline \multirow{2}{*}{ PAT 3 } & *** & *** & \\
& 234.92 & 342.65 & -1.00 \\
\hline
\end{tabular}

c) Extension (1984-2000) : Cumulative Survival Rate

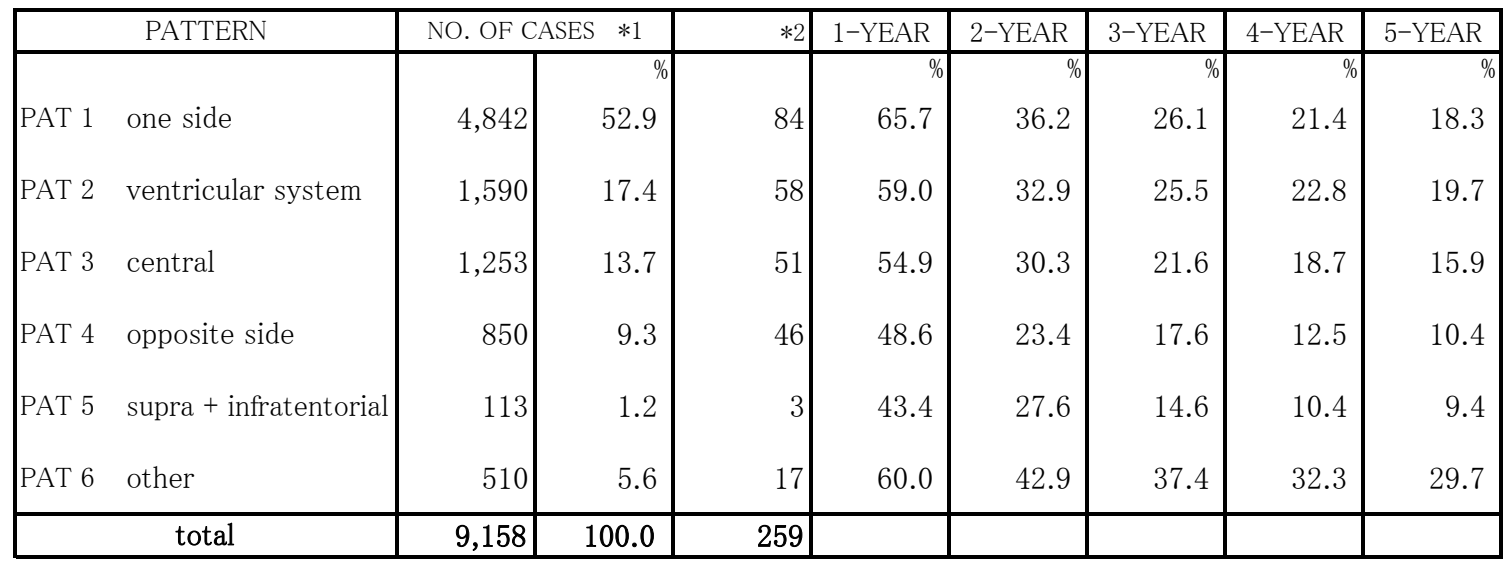

*1 Number of cases who lived more than 30 days.

$* 2$ Number of cases who died within 30 days.

\section{Mantel Chi-Square Test}

\begin{tabular}{|c|c|c|c|c|c|c|}
\hline & PAT 1 & PAT 2 & PAT 3 & PAT 4 & PAT 5 & PAT 6 \\
\hline \multirow{2}{*}{ PAT 1} & & & & *** & $* *$ & \\
\hline & -1.00 & 1.01 & 9.98 & 32.28 & 7.32 & 5.64 \\
\hline \multirow{2}{*}{ PAT 2} & & & & *** & & \\
\hline & 1.01 & -1.00 & 3.11 & 14.90 & 4.73 & 6.56 \\
\hline \multirow{2}{*}{ PAT 3} & & & & & & *** \\
\hline & 9.98 & 3.11 & -1.00 & 5.08 & 1.96 & 14.18 \\
\hline \multirow{2}{*}{ PAT 4} & *** & $* * *$ & & & & *** \\
\hline & 32.28 & 14.90 & 5.08 & -1.00 & 0.14 & 26.38 \\
\hline \multirow{2}{*}{ PAT 5} & $* *$ & & & & & *** \\
\hline & 7.32 & 4.73 & 1.96 & 0.14 & -1.00 & 10.32 \\
\hline \multirow{2}{*}{ PAT 6} & $*$ & & *** & $* * *$ & $* * *$ & \\
\hline & 5.64 & 6.56 & 14.18 & 26.38 & 10.32 & -1.00 \\
\hline
\end{tabular}


d) Mode of Oparation (1984-2000): Cumulative Survival Rate

\begin{tabular}{|ll|r|r|r|r|r|r|r|r|}
\hline & PATTERN & \multicolumn{2}{|c|}{ NO. OF CASES $* 1$} & $* 2$ & 1-YEAR & 2-YEAR & 3-YEAR & 4-YEAR & 5-YEAR \\
\hline PAT 0 & none & 577 & 6.2 & 40 & 45.5 & 22.6 & 17.5 & 14.5 & 13.2 \\
PAT 1 & decompression & 159 & 1.7 & 14 & 56.4 & 34.5 & 21.4 & 15.5 & 13.3 \\
PAT 2 & biopsy or partial & 2,210 & 23.9 & 77 & 48.2 & 23.9 & 16.4 & 13.4 & 11.0 \\
PAT 3 & $50 \%$ resected & 1,041 & 11.3 & 43 & 52.0 & 23.8 & 15.2 & 12.2 & 10.8 \\
PAT 4 & 75\% resected & 2,217 & 24.0 & 58 & 61.5 & 31.9 & 22.6 & 0.2 & 15.5 \\
PAT 5 & 95\% resected & 2,158 & 23.4 & 22 & 73.2 & 44.1 & 34.8 & 28.3 & 23.9 \\
PAT 6 & total removal & 872 & 9.4 & 11 & 81.5 & 58.9 & 49.2 & 45.4 & 42.8 \\
\hline \multicolumn{2}{c}{ total } & 9,234 & 100.0 & 265 & & & & & \\
\hline
\end{tabular}

*1 Number of cases who lived more than 30 days.

$* 2$ Number of cases who died within 30 days.

\section{Mantel Chi-Square Test}

\begin{tabular}{|l|r|r|r|r|r|r|r|}
\hline & PAT 0 & PAT 1 & PAT 2 & PAT 3 & PAT 4 & PAT 5 & PAT 6 \\
\hline PAT 0 & -1.00 & 0.68 & 0.00 & 0.08 & 8.84 & 50.41 & 107.45 \\
\hline PAT 1 & 0.68 & -1.00 & 1.13 & 0.90 & 0.35 & 9.49 & 39.37 \\
\hline PAT 2 & 0.00 & 1.13 & -1.00 & 0.15 & 20.94 & 112.60 & 187.89 \\
\hline PAT 3 & 0.08 & 0.90 & 0.15 & -1.00 & 11.35 & 72.44 & 144.83 \\
\hline PAT 4 & $* * * * * *$ & $* * *$ \\
& 8.84 & 0.35 & 20.94 & 11.35 & -1.00 & 38.85 & 120.94 \\
\hline PAT 5 & $* * *$ & $* *$ & $* * *$ & $* * *$ & $* * *$ & & $* * *$ \\
\hline PAT 6 & 50.41 & 9.49 & 112.60 & 72.44 & 38.85 & -1.00 & 46.35 \\
\hline
\end{tabular}

\section{e) Radiation Therapy (1984-2000) : Cumulative Survival Rate}

\begin{tabular}{|c|c|c|c|c|c|c|c|c|}
\hline PATTERN & \multicolumn{2}{|c|}{ NO. OF CASES $* 1$} & $* 2$ & 1-YEAR & 2-YEAR & 3-YEAR & 4-YEAR & 5-YEAR \\
\hline & & $\%$ & & 0 & $\%$ & $\%$ & $\%$ & $\%$ \\
\hline none & 1,254 & 13.4 & 228 & 40.7 & 27.1 & 22.7 & 21.1 & 20.4 \\
\hline irradiated & 8,070 & 86.6 & 39 & 63.9 & 35.2 & 25.8 & 21.2 & 18.1 \\
\hline total & 9,324 & 100.0 & 267 & & & & & \\
\hline
\end{tabular}

$* 1$ Number of cases who lived more than 30 days.

$* 2$ Number of cases who died within 30 days.

Mantel Chi-Square Test : Significant by $p<0.001$ 
f) Clinical Grading (1984-2000) : Cumulative Survival Rate

\begin{tabular}{|c|c|c|c|c|c|c|c|c|}
\hline PATTERN & NO. OF & $\mathrm{SES} * 1$ & $* 2$ & 1-YEAR & 2-YEAR & 3-YEAR & 4-YEAR & 5-YEAR \\
\hline & & $\%$ & & $\%$ & $\%$ & $\%$ & $\%$ & $\%$ \\
\hline PAT 1 no symptoms & 140 & 1.5 & 2 & 80.8 & 65.2 & 56.6 & 46.6 & 41.8 \\
\hline PAT 2 complaints only & 776 & 8.4 & 11 & 74.0 & 49.8 & 40.2 & 33.5 & 27.1 \\
\hline PAT 3 focal signs & 5,114 & 55.2 & 81 & 60.9 & 32.6 & 23.0 & 19.1 & 16.7 \\
\hline PAT 4 increased ICP & 2,157 & 23.3 & 52 & 63.4 & 37.1 & 28.9 & 24.4 & 21.3 \\
\hline PAT $5 \begin{array}{l}\text { disturbance of } \\
\text { consciousness }\end{array}$ & 966 & 10.4 & 83 & 42.3 & 18.0 & 12.5 & 10.3 & 8.7 \\
\hline PAT 6 coma & 95 & 1.0 & 27 & 40.1 & 23.8 & 23.8 & 16.2 & 16.2 \\
\hline $\begin{array}{ll}\text { PAT } 7 & \begin{array}{l}\text { respiratory } \\
\text { impairment }\end{array}\end{array}$ & 17 & 0.2 & 5 & 51.5 & 19.3 & 12.9 & 12.9 & 12.9 \\
\hline total & 9,265 & 100.0 & 261 & & & & & \\
\hline
\end{tabular}

*1 Number of cases who lived more than 30 days.

$* 2$ Number of cases who died within 30 days.

\section{Mantel Chi-Square Test}

\begin{tabular}{|c|c|c|c|c|c|c|c|}
\hline & PAT 1 & PAT 2 & PAT 3 & PAT 4 & PAT 5 & PAT 6 & PAT 7 \\
\hline \multirow{2}{*}{ PAT 1} & & & *** & $* * *$ & $* * *$ & $* * *$ & $* *$ \\
\hline & -1.00 & 6.45 & 25.61 & 17.08 & 45.47 & 16.05 & 6.71 \\
\hline \multirow{2}{*}{ PAT 2} & & & *** & *** & *** & *** & \\
\hline & 6.45 & -1.00 & 33.91 & 13.02 & 80.97 & 12.60 & 2.71 \\
\hline \multirow{2}{*}{ PAT 3} & *** & *** & & $* *$ & *** & & \\
\hline & 25.61 & 33.91 & -1.00 & 8.44 & 40.30 & 2.22 & 0.30 \\
\hline \multirow{2}{*}{ PAT 4} & *** & *** & ** & & $* * *$ & & \\
\hline & 17.08 & 13.02 & 8.44 & -1.00 & 53.56 & 4.12 & 0.71 \\
\hline \multirow{2}{*}{ PAT 5} & *** & *** & *** & *** & & & \\
\hline & 45.47 & 80.97 & 40.30 & 53.56 & -1.00 & 0.39 & 0.13 \\
\hline \multirow{2}{*}{ PAT 6} & *** & *** & & $*$ & & & \\
\hline & 16.05 & 12.60 & 2.22 & 4.12 & 0.39 & -1.00 & 0.01 \\
\hline PAT 7 & $\begin{array}{r}* * \\
6.71\end{array}$ & 2.71 & 0.30 & 0.71 & 0.13 & 0.01 & -1.00 \\
\hline
\end{tabular}


9) Glioblastoma

a) Operation(1984-2000): Cumulative Survival Rate

\begin{tabular}{|ll|r|r|r|r|r|r|r|r|}
\hline & PATTERN & \multicolumn{2}{|c|}{ NO. OF CASES $* 1$} & $* 2$ & 1-YEAR & 2-YEAR & 3-YEAR & 4-YEAR & 5-YEAR \\
\hline PAT 0 none & & $\%$ & & $\%$ & $\%$ & $\%$ & $\%$ & $\%$ \\
PAT 1 decompression & 360 & 6.8 & 28 & 38.7 & 14.6 & 8.6 & 7.4 & 6.8 \\
PAT 2 biopsy or partial & 1,203 & 22.6 & 47 & 39.6 & 14.7 & 7.5 & 6.1 & 4.0 \\
PAT 3 50\% resected & 661 & 12.4 & 31 & 45.1 & 14.9 & 6.7 & 5.7 & 4.1 \\
PAT 4 75\% resected & 1,363 & 25.6 & 35 & 53.9 & 18.9 & 10.7 & 8.0 & 6.2 \\
PAT 5 95\% resected & 1,256 & 23.6 & 13 & 66.1 & 28.9 & 20.2 & 14.1 & 11.3 \\
PAT 6 total removal & 415 & 7.8 & 7 & 72.3 & 35.8 & 23.4 & 20.6 & 18.3 \\
\hline \multicolumn{1}{c}{ total } & 5,328 & 100.0 & 166 & & & & & \\
\hline
\end{tabular}

*1 Number of cases who lived more than 30 days.

*2 Number of cases who died within 30 days.

\section{Mantel Chi-Square Test}

\begin{tabular}{|l|r|r|r|r|r|r|r|}
\hline & PAT 0 & PAT 1 & PAT 2 & PAT 3 & PAT 4 & PAT 5 & PAT 6 \\
\hline PAT 0 & -1.00 & 0.00 & 0.05 & 0.12 & 3.79 & 23.23 & 26.78 \\
\hline PAT 1 & 0.00 & -1.00 & 0.01 & 0.03 & 0.89 & 5.98 & 10.61 \\
\hline PAT 2 & 0.05 & 0.01 & -1.00 & 0.43 & 9.69 & 51.13 & 44.31 \\
\hline PAT 3 & 0.12 & 0.03 & 0.43 & -1.00 & 3.80 & 30.83 & 33.32 \\
\hline PAT 4 & 3.79 & 0.89 & 9.69 & 3.80 & -1.00 & 18.71 & 24.51 \\
\hline PAT 5 & *** & * & *** & *** & *** & *** \\
\hline PAT 6 & 23.23 & 5.98 & 51.13 & 30.83 & 18.71 & -1.00 & 4.30 \\
\hline & 26.78 & 10.61 & 44.31 & 33.32 & 24.51 & 4.30 & -1.00 \\
\hline
\end{tabular}

\section{b) Radiation Therapy (1984-2000): Cumulative Survival Rate}

\begin{tabular}{|l|r|r|r|r|r|r|r|r|}
\hline PATTERN & \multicolumn{2}{|l|}{ NO. OF CASES $* 1$} & $* 2$ & 1-YEAR & 2-YEAR & 3-YEAR & 4-YEAR & 5-YEAR \\
\hline PAT 0 none & 746 & 13.8 & 141 & 29.1 & 13.4 & 8.9 & 7.8 & 7.1 \\
PAT 1 irradiated & 4,649 & 86.2 & 26 & 56.6 & 22.2 & 13.2 & 10.0 & 7.7 \\
\hline total & 5,395 & 100.0 & 167 & & & & & \\
\hline
\end{tabular}

$* 1$ Number of cases who lived more than 30 days.

$* 2$ Number of cases who died within 30 days.

Mantel Chi-Square Test : Significant by $p<0.001$ 
c) Clinical Grading (1984-2000): Cumulative Survival Rate

\begin{tabular}{|c|c|c|c|c|c|c|c|c|c|}
\hline & PATTERN & \multicolumn{2}{|c|}{ NO. OF CASES $* 1$} & $* 2$ & 1-YEAR & 2-YEAR & 3-YEAR & 4-YEAR & 5-YEAR \\
\hline & & & $\%$ & & $\%$ & $\%$ & $\%$ & $\%$ & $\%$ \\
\hline PAT 1 & no symptoms & 64 & 1.2 & 0 & 69.4 & 41.2 & 33.3 & 25.9 & 17.2 \\
\hline PAT 2 & complaints only & 412 & 7.7 & 9 & 62.6 & 29.7 & 20.7 & 15.1 & 8.7 \\
\hline PAT 3 & focal signs & 3,065 & 57.1 & 50 & 54.7 & 21.4 & 12.2 & 9.6 & 7.8 \\
\hline PAT 4 & increased ICP & 1,127 & 21.0 & 28 & 52.6 & 21.1 & 13.2 & 9.7 & 8.4 \\
\hline PAT 5 & $\begin{array}{l}\text { disturbance of } \\
\text { consciousness }\end{array}$ & 628 & 11.7 & 60 & 37.7 & 11.2 & 4.8 & 3.9 & 2.7 \\
\hline PAT 6 & coma & 57 & 1.1 & 14 & 32.7 & 11.9 & 11.9 & 5.9 & 5.9 \\
\hline PAT 7 & $\begin{array}{l}\text { respiratory } \\
\text { impairment }\end{array}$ & 11 & 0.2 & 3 & 54.5 & 18.2 & 9.1 & 9.1 & 9.1 \\
\hline & total & 5,364 & 100.0 & 164 & & & & & \\
\hline
\end{tabular}

$* 1$ Number of cases who lived more than 30 days.

$* 2$ Number of cases who died within 30 days.

Mantel Chi-Square Test

\begin{tabular}{|c|c|c|c|c|c|c|c|}
\hline & PAT 1 & PAT 2 & PAT 3 & PAT 4 & PAT 5 & PAT 6 & PAT 7 \\
\hline PAT 1 & -1.00 & $\begin{array}{r}* * \\
9.32\end{array}$ & $\begin{array}{r}* \\
4.99\end{array}$ & 2.40 & $\begin{array}{r}* * * \\
62.80\end{array}$ & 1.08 & -1.00 \\
\hline PAT 2 & $\begin{array}{r}* * \\
9.32 \\
\end{array}$ & -1.00 & $\begin{array}{r}* * * * \\
21.85 \\
\end{array}$ & 1.42 & $\begin{array}{r}* * * * \\
64.53 \\
\end{array}$ & $\begin{array}{r}* \\
6.12 \\
\end{array}$ & -1.00 \\
\hline PAT 3 & $\begin{array}{r}* \\
4.99 \\
\end{array}$ & $\begin{array}{r}* * * \\
21.85\end{array}$ & -1.00 & $\begin{array}{r}* * * \\
10.71\end{array}$ & $\begin{array}{r}* * * \\
43.99 \\
\end{array}$ & 0.02 & -1.00 \\
\hline PAT 4 & 2.40 & 1.42 & $\begin{array}{r}* * * * \\
10.71 \\
\end{array}$ & -1.00 & $\begin{array}{r}* * * \\
48.74\end{array}$ & 2.30 & -1.00 \\
\hline PAT 5 & $\begin{array}{r}* * * \\
62.80 \\
\end{array}$ & $\begin{array}{r}* * * * \\
64.53 \\
\end{array}$ & $\begin{array}{r}* * * \\
43.99 \\
\end{array}$ & $\begin{array}{r}* * * \\
48.74 \\
\end{array}$ & -1.00 & $\begin{array}{r}* * * * \\
10.47 \\
\end{array}$ & -1.00 \\
\hline PAT 6 & 1.08 & $\begin{array}{r}* * \\
6.12 \\
\end{array}$ & 0.02 & 2.30 & $\begin{array}{r}* * * * \\
10.47 \\
\end{array}$ & -1.00 & -1.00 \\
\hline PAT 7 & -1.00 & -1.00 & -1.00 & -1.00 & -1.00 & -1.00 & -1.00 \\
\hline
\end{tabular}


d) Oparation With or Without Radiation Therapy (1984-2000): Cumulative Survival Rate

\begin{tabular}{|c|c|c|c|c|c|c|c|c|c|}
\hline & PATTERN & \multicolumn{2}{|c|}{ NO. OF CASES $* 1$} & $* 2$ & 1-YEAR & 2-YEAR & 3-YEAR & 4-YEAR & 5-YEAR \\
\hline & & & $\%$ & & $\%$ & $\%$ & $\%$ & $\%$ & $\%$ \\
\hline PAT 1 & ope (biopsy) + radiation & 1,047 & 21.5 & 10 & 43.0 & 16.3 & 8.6 & 6.9 & 4.5 \\
\hline PAT 2 & ope (biopsy) alone & 152 & 3.1 & 37 & 15.9 & 3.6 & 0.0 & - & - \\
\hline PAT 3 & $\begin{array}{l}\text { ope }(50-75 \% \text { resected }) \\
+ \text { radiation }\end{array}$ & 1,773 & 36.3 & 6 & 54.2 & 18.5 & 9.7 & 7.4 & 5.5 \\
\hline PAT 4 & $\begin{array}{l}\text { ope }(50-75 \% \\
\text { resected) alone }\end{array}$ & 244 & 5.0 & 60 & 26.8 & 11.2 & 7.8 & 6.7 & 6.7 \\
\hline PAT 5 & $\begin{array}{l}\text { ope }(\geqq 95 \% \text { resected }) \\
+ \text { radiation }\end{array}$ & 1,482 & 30.4 & 5 & 71.0 & 31.7 & 21.6 & 15.9 & 13.0 \\
\hline PAT 6 & $\begin{array}{l}\text { ope }(\geqq 95 \% \\
\text { resected) alone }\end{array}$ & 181 & 3.7 & 14 & 39.6 & 24.4 & 17.6 & 15.3 & 15.3 \\
\hline PAT 7 & $\begin{array}{l}\text { ope (all cases) }+ \\
\text { radiation }\end{array}$ & 4,358 & 88.1 & 21 & 57.1 & 22.4 & 13.4 & 10.1 & 7.8 \\
\hline PAT 8 & ope alone (all cases) & 591 & 11.9 & 116 & 27.8 & 13.3 & 9.0 & 7.9 & 7.5 \\
\hline
\end{tabular}

*1 Number of cases who lived more than 30 days.

*2 Number of cases who died within 30 days.

\section{Mantel Chi-Square Test}

\begin{tabular}{|c|c|c|c|c|c|c|c|c|}
\hline & PAT 1 & PAT 2 & PAT 3 & PAT 4 & PAT 5 & PAT 6 & PAT 7 & PAT 8 \\
\hline \multirow{2}{*}{ PAT 1} & & ** & $*$ & & $* * *$ & & *** & \\
\hline & -1.00 & 9.32 & 4.99 & 2.40 & 62.80 & 1.08 & 17.43 & 2.32 \\
\hline \multirow{2}{*}{ PAT 2} & & & $* * *$ & & $* * *$ & & $* * *$ & \\
\hline & 9.32 & -1.00 & 21.85 & 1.42 & 64.53 & 6.12 & 29.62 & 2.28 \\
\hline \multirow{2}{*}{ PAT 3} & & $* * *$ & & *** & $* * *$ & & $*$ & *** \\
\hline & 4.99 & 21.85 & -1.00 & 10.71 & 43.99 & 0.02 & 3.95 & 15.18 \\
\hline \multirow{2}{*}{ PAT 4} & & & $* * *$ & & $* * *$ & & $* * *$ & \\
\hline & 2.40 & 1.42 & 10.71 & -1.00 & 48.74 & 2.30 & 17.81 & 0.04 \\
\hline \multirow{2}{*}{ PAT 5} & *** & $* * *$ & *** & *** & & *** & *** & *** \\
\hline & 62.80 & 64.53 & 43.99 & 48.74 & -1.00 & 10.47 & 32.54 & 74.31 \\
\hline PAT 6 & 1.08 & $\begin{array}{r}* \\
6 \\
6\end{array}$ & 0.02 & 2.30 & $\begin{array}{r}* * * \\
1047\end{array}$ & -100 & 0.69 & 248 \\
\hline \multirow{2}{*}{ PAT 7} & $* * *$ & $* * *$ & & $* * *$ & *** & & & $* * *$ \\
\hline & 17.43 & 29.62 & 3.95 & 17.81 & 32.54 & 0.69 & -1.00 & 30.97 \\
\hline PAT 8 & 2.32 & 2.28 & $\begin{array}{r}* * * \\
15.18\end{array}$ & 0.04 & $\begin{array}{r}* * * * \\
74.31\end{array}$ & 2.48 & $\begin{array}{r}\text { *** } \\
30.97\end{array}$ & -1.00 \\
\hline
\end{tabular}


e) Chemotherapy (1984-2000): Cumulative Survival Rate

\begin{tabular}{|c|c|c|c|c|c|c|c|c|}
\hline PATTERN & NO. OF C & $* 1$ & $* 2$ & $1-Y E A R$ & 2-YEAR & 3-YEAR & 4-YEAR & 5-YEAR \\
\hline & & $\%$ & & $\%$ & $\%$ & $\%$ & $\%$ & $\%$ \\
\hline PAT 1 chemo(-) & 1,582 & 31.7 & 142 & 38.9 & 14.5 & 8.8 & 7.6 & 6.7 \\
\hline PAT 2 BLM & 29 & 0.6 & 0 & 57.9 & 23.2 & 13.9 & 13.9 & 9.3 \\
\hline PAT 3 ACNU & 2,762 & 55.4 & 16 & 58.7 & 24.3 & 14.3 & 11.1 & 8.8 \\
\hline PAT $4 \quad$ ACNU + VCR & 612 & 12.3 & 3 & 59.8 & 25.0 & 12.4 & 8.9 & 7.2 \\
\hline total & 4,985 & 100.0 & 161 & & & & & \\
\hline
\end{tabular}

*1 Number of cases who lived more than 30 days.

$* 2$ Number of cases who died within 30 days.

\section{Mantel Chi-Square Test}

\begin{tabular}{|l|r|r|r|r|}
\hline & PAT 1 & PAT 2 & PAT 3 & PAT 4 \\
\hline PAT 1 & -1.00 & 0.66 & 33.08 & $\begin{array}{r}* * * \\
* * *\end{array}$ \\
\hline PAT 2 & 0.66 & -1.00 & 0.00 & 0.00 \\
\hline PAT 3 & $\begin{array}{r}* * * \\
33.08\end{array}$ & 0.00 & -1.00 & 0.03 \\
\hline PAT 4 & $\begin{array}{r}* * * \\
\text { PT }\end{array}$ & 0.00 & 0.03 & -1.00 \\
\hline
\end{tabular}

f) Extension (1984-2000) : Cumulative Survival Rate

\begin{tabular}{|c|c|c|c|c|c|c|c|c|c|}
\hline & PATTERN & NO. OF CA & S $\quad * 1$ & $* 2$ & 1-YEAR & 2-YEAR & 3-YEAR & 4-YEAR & 5-YEAR \\
\hline & & & $\%$ & & $\%$ & $\%$ & $\%$ & $\%$ & $\%$ \\
\hline PAT 1 & one side & 3,069 & 57.7 & 56 & 59.7 & 24.9 & 15.4 & 11.8 & 9.5 \\
\hline PAT 2 & venricular system & 824 & 15.5 & 34 & 47.1 & 14.9 & 8.9 & 6.8 & 5.0 \\
\hline PAT 3 & central & 684 & 12.9 & 33 & 45.6 & 17.4 & 8.1 & 6.9 & 5.1 \\
\hline PAT 4 & opposite side & 551 & 10.4 & 35 & 40.7 & 13.5 & 8.2 & 5.5 & 4.0 \\
\hline PAT 5 & $\begin{array}{l}\text { supra }+ \\
\text { infratentorial }\end{array}$ & 48 & 0.9 & 3 & 27.5 & 16.5 & 8.2 & 2.7 & 2.7 \\
\hline PAT 6 & other & 140 & 2.6 & 4 & 37.0 & 18.7 & 15.7 & 11.4 & 7.4 \\
\hline & total & 5,316 & 100.0 & 165 & & & & & \\
\hline
\end{tabular}

*1 Number of cases who lived more than 30 days.

$* 2$ Number of caes who died within 30 days. 
Mantel Chi-Square Test

\begin{tabular}{|c|c|c|c|c|c|c|}
\hline & PAT 1 & PAT 2 & PAT 3 & PAT 4 & PAT 5 & PAT 6 \\
\hline \multirow{2}{*}{ PAT 1} & & *** & $* * *$ & *** & $*$ & $*$ \\
\hline & -1.00 & 16.71 & 14.75 & 21.83 & 5.07 & 4.44 \\
\hline \multirow[b]{2}{*}{ PAT 2} & $* * *$ & & & & & \\
\hline & 16.71 & -1.00 & 0.00 & 0.96 & 1.10 & 0.03 \\
\hline \multirow{2}{*}{ PAT 3} & $* * *$ & & & & & \\
\hline & 14.75 & 0.00 & -1.00 & 0.84 & 0.91 & 0.02 \\
\hline PAT 4 & $\begin{array}{r}* * * \\
21.83\end{array}$ & 0.96 & 0.84 & -1.00 & 0.34 & 0.20 \\
\hline PAT 5 & $\begin{array}{r}* \\
5.07\end{array}$ & 1.10 & 0.91 & 0.34 & -1.00 & 0.54 \\
\hline PAT 6 & $\begin{array}{r}* \\
4.44\end{array}$ & 0.03 & 0.02 & 0.20 & 0.54 & -1.00 \\
\hline
\end{tabular}


10) Low Grade Glioma (Histology Code $1,3,7,8,10,13,15)$

a) Location (1984-2000): Cumulative Survival Rate

\begin{tabular}{|c|c|c|c|c|c|c|c|c|c|}
\hline & PATTERN & NO. OF C & $* 1$ & $* 2$ & 1-YEAR & 2-YEAR & 3-YEAR & 4-YEAR & 5-YEAR \\
\hline PAT 1 & frontal lobe & 2,066 & 36.9 & 21 & 90.6 & 82.4 & 75.8 & 72.2 & 68.1 \\
\hline PAT 2 & temporal lobe & 828 & 14.8 & 10 & 88.9 & 77.9 & 71.6 & 68.5 & 62.9 \\
\hline PAT 3 & parietal lobe & 537 & 9.6 & 9 & 89.2 & 77.6 & 71.3 & 66.5 & 64.2 \\
\hline PAT 4 & occipital lobe & 129 & 2.3 & 1 & 88.7 & 77.3 & 73.4 & 72.4 & 70.1 \\
\hline PAT 5 & lateral ventricle & 221 & 3.9 & 3 & 92.1 & 91.1 & 91.1 & 89.8 & 87.5 \\
\hline PAT 6 & $\begin{array}{l}\text { chiasmal region, optic } \\
\text { nerve }\end{array}$ & 189 & 3.4 & 1 & 97.2 & 95.4 & 94.1 & 93.4 & 92.6 \\
\hline PAT 7 & $\begin{array}{l}\text { third ventricle, ant. \& } \\
\text { post. }\end{array}$ & 171 & 3.1 & 3 & 87.7 & 83.7 & 81.5 & 76.8 & 75.0 \\
\hline PAT 8 & $\begin{array}{l}\text { rostral brain stem and } \\
\text { basal ganglia }\end{array}$ & 280 & 5.0 & 6 & 81.3 & 0.6 & 53.8 & 50.9 & 47.6 \\
\hline PAT 9 & pineal region & 40 & 0.7 & 0 & 94.7 & 85.8 & 85.8 & 82.1 & 82.1 \\
\hline PAT10 & aqueduct & 21 & 0.4 & 0 & 100.0 & 94.7 & 94.7 & 88.2 & 88.2 \\
\hline PAT11 & caudal brain stem & 294 & 5.3 & 5 & 69.6 & 49.7 & 41.4 & 39.8 & 36.8 \\
\hline PAT12 & cerebellar vermis & 220 & 3.9 & 3 & 0.9 & 88.4 & 86.2 & 84.5 & 82.6 \\
\hline PAT13 & $\begin{array}{l}\text { cerebellar } \\
\text { hemisphere }\end{array}$ & 311 & 5.6 & 8 & 93.6 & 87.4 & 85.4 & 84.5 & 84.0 \\
\hline PAT14 & fourth ventricle & 288 & 5.1 & 7 & 93.1 & 85.5 & 79.9 & 74.8 & 70.8 \\
\hline & total & 5,595 & 100.0 & 77 & & & & & \\
\hline
\end{tabular}

*1 Number of cases who lived more than 30 days.

$* 2$ Number of cases who died within 30 days.

Mantel Chi-Square Test

\begin{tabular}{|c|c|c|c|c|c|c|c|c|c|c|c|c|c|c|}
\hline & PAT 1 & PAT 2 & PAT 3 & PAT 4 & PAT 5 & PAT 6 & PAT 7 & PAT 8 & PAT 9 & PAT 10 & PAT 11 & PAT 12 & PAT 13 & PAT 14 \\
\hline \multirow{2}{*}{ PAT 1} & & & & & *** & *** & & $* * *$ & & & *** & *** & *** & \\
\hline & -1.00 & 5.10 & 2.86 & 0.01 & 22.29 & 33.68 & 1.67 & 40.96 & 1.98 & 2.86 & 107.57 & 12.81 & 19.02 & 0.91 \\
\hline \multirow{2}{*}{ PAT 2} & & & & & *** & *** & & $* * *$ & & & *** & *** & *** & \\
\hline & 5.10 & -1.00 & 0.04 & 1.05 & 30.23 & 42.14 & 4.88 & 17.28 & 3.40 & 3.93 & 53.99 & 19.63 & 27.87 & 4.73 \\
\hline \multirow{2}{*}{ PAT 3} & & & & & $* * *$ & *** & & $* * *$ & & & *** & *** & *** & \\
\hline & 2.86 & 0.04 & -1.00 & 0.73 & 27.37 & 38.94 & 4.02 & 15.89 & 3.18 & 3.76 & 46.37 & 16.98 & 24.11 & 3.60 \\
\hline \multirow{2}{*}{ PAT 4} & & & & & *** & *** & & *** & & & $* * *$ & & $* *$ & \\
\hline & 0.01 & 1.05 & 0.73 & -1.00 & 12.43 & 22.56 & 0.70 & 10.73 & 1.59 & 2.50 & 23.27 & 5.76 & 7.98 & 0.25 \\
\hline \multirow{2}{*}{ PAT 5} & $* * *$ & **** & $* * *$ & $* * *$ & & & ** & $* * *$ & & & $* * *$ & & & *** \\
\hline & 22.29 & 30.23 & 27.37 & 12.43 & -1.00 & 2.44 & 7.64 & 57.56 & 0.71 & 0.03 & 85.53 & 1.63 & 1.19 & 12.85 \\
\hline \multirow{2}{*}{ PAT 6} & *** & *** & *** & *** & & & $* * *$ & *** & & & $* * *$ & ** & ** & $* * *$ \\
\hline & 33.68 & 42.14 & 38.94 & 22.56 & 2.44 & -1.00 & 16.57 & 69.64 & 3.60 & 0.26 & 95.59 & 7.53 & 6.75 & 23.83 \\
\hline \multirow{2}{*}{ PAT 7} & & & & & ** & $* * *$ & & $* * *$ & & & $* * *$ & & $*$ & \\
\hline & 1.67 & 4.88 & 4.02 & 0.70 & 7.64 & 16.57 & -1.00 & 20.21 & 0.60 & 1.56 & 38.26 & 2.57 & 4.01 & 0.26 \\
\hline \multirow{2}{*}{ PAT 8} & *** & *** & $* * *$ & $* * *$ & $* * *$ & $* * *$ & $* * *$ & & $* *$ & ** & & *** & *** & *** \\
\hline & 40.96 & 17.28 & 15.89 & 10.73 & 57.56 & 69.64 & 20.21 & -1.00 & 9.23 & 7.85 & 5.49 & 43.76 & 58.34 & 25.77 \\
\hline PAT 9 & 1.98 & 3.40 & 3.18 & 1.59 & 0.71 & 3.60 & 0.60 & $\begin{array}{r}* * \\
9.23\end{array}$ & -1.00 & 0.48 & $\begin{array}{r}* * * \\
14.75\end{array}$ & 0.01 & 0.04 & 1.12 \\
\hline \multirow{2}{*}{ PAT10 } & & $*$ & & & & & & **1 & & & $* * *$ & & & \\
\hline & 2.86 & 3.93 & 3.76 & 2.50 & 0.03 & 0.26 & 1.56 & 7.85 & 0.48 & -1.00 & 11.24 & 0.48 & 0.38 & 2.21 \\
\hline \multirow{2}{*}{ PAT11 } & **** & **** & $\begin{array}{l}* * * \\
\end{array}$ & F** & $* * *$ & *** & *** & & *** & **** & & **** & *** & *** \\
\hline & 107.57 & 53.99 & 46.37 & 23.27 & 85.53 & 95.59 & 38.26 & 5.49 & 14.75 & 11.24 & -1.00 & 69.95 & 92.52 & 53.62 \\
\hline \multirow{2}{*}{ PAT12 } & *** & *** & *** & & & $* *$ & & $* * *$ & & & & & & \\
\hline & 12.81 & 19.63 & 16.98 & 5.76 & 1.63 & 7.53 & 2.57 & 43.76 & 0.01 & 0.48 & 69.95 & -1.00 & 0.08 & 5.82 \\
\hline PAT13 & *** & $\begin{array}{r}* * * \\
87\end{array}$ & *** & ** & 1.10 & $\begin{array}{r}* * \\
75\end{array}$ & $*$ & $* * *$ & 04 & 28 & *** & 0 & & \\
\hline \multirow[b]{2}{*}{ PAT14 } & & $\begin{array}{r}21.01 \\
*\end{array}$ & & & 1.19 & 0.15 & 4.01 & $\frac{50.04}{* * *}$ & .04 & 0.00 & $\frac{9 Z . J Z}{* * *}$ & *.00 & * & 0.00 \\
\hline & 0.91 & 4.73 & 3.60 & 0.25 & 12.85 & 23.83 & 0.26 & 25.77 & 1.12 & 2.21 & 53.62 & 5.82 & 8.30 & -1.00 \\
\hline
\end{tabular}


b) Operation (1984-2000): Cumulative Survival Rate

\begin{tabular}{|c|c|c|c|c|c|c|c|c|c|}
\hline \multicolumn{2}{|r|}{ PATTERN } & \multicolumn{2}{|c|}{ NO. OF CASES } & \multirow[t]{2}{*}{$* 2$} & \multirow{2}{*}{$\frac{1 \text {-YEAR }}{\%}$} & \multirow{2}{*}{$\frac{2-Y E A R}{\%}$} & \multirow{2}{*}{$\frac{3 \text {-YEAR }}{\%}$} & \multirow{2}{*}{$\frac{4-\mathrm{YEAR}}{\%}$} & \multirow{2}{*}{$\frac{5 \text {-YEAR }}{\%}$} \\
\hline & & & $\%$ & & & & & & \\
\hline PAT 0 & none & 334 & 5.8 & 6 & 78.1 & 64.5 & 55.5 & 51.6 & 49.2 \\
\hline PAT 1 & decompression & 107 & 1.9 & 6 & 84.2 & 68.2 & 63.0 & 61.6 & 61.6 \\
\hline PAT 2 & biopsy or partial & 1,606 & 27.9 & 23 & 82.5 & 70.0 & 63.3 & 59.8 & 56.6 \\
\hline PAT 3 & $50 \%$ resected & 503 & 8.7 & 8 & 87.6 & 78.6 & 72.7 & 67.2 & 63.1 \\
\hline PAT 4 & $75 \%$ resected & 1,005 & 17.5 & 18 & 90.4 & 80.1 & 72.9 & 70.0 & 64.5 \\
\hline PAT 5 & $95 \%$ resected & 1,139 & 19.8 & 11 & 94.7 & 87.8 & 84.3 & 80.9 & 77.6 \\
\hline PAT 6 & total removal & 1,055 & 18.4 & 9 & 98.0 & 95.2 & 92.9 & 91.4 & 90.1 \\
\hline & total & 5,749 & 100.0 & 81 & & & & & \\
\hline
\end{tabular}

*1 Number of cases who lived more than 30 days.

$* 2$ Number of cases who died within 30 days.

\section{Mantel Chi-Square Test}

\begin{tabular}{|c|c|c|c|c|c|c|c|}
\hline & PAT 0 & PAT 1 & PAT 2 & PAT 3 & PAT 4 & PAT 5 & PAT 6 \\
\hline \multirow{2}{*}{ PAT 0} & & & & *** & *** & *** & *** \\
\hline & -1.00 & 2.34 & 4.17 & 13.70 & 24.38 & 89.42 & 226.21 \\
\hline \multirow{2}{*}{ PAT 1} & & & & & & *** & *** \\
\hline & 2.34 & -1.00 & 0.34 & 0.50 & 1.32 & 15.51 & 71.76 \\
\hline \multirow{2}{*}{ PAT 2} & $*$ & & & $*$ & *** & *** & *** \\
\hline & 4.17 & 0.34 & -1.00 & 6.34 & 16.85 & 103.00 & 252.66 \\
\hline \multirow{2}{*}{ PAT 3} & *** & & $*$ & & & *** & *** \\
\hline & 13.70 & 0.50 & 6.34 & -1.00 & 0.42 & 30.32 & 132.98 \\
\hline \multirow{2}{*}{ PAT 4} & *** & & *** & & & *** & *** \\
\hline & 24.38 & 1.32 & 16.85 & 0.42 & -1.00 & 33.18 & 146.72 \\
\hline \multirow{2}{*}{ PAT 5} & *** & *** & *** & *** & *** & & *** \\
\hline & 89.42 & 15.51 & 103.00 & 30.32 & 33.18 & -1.00 & 48.39 \\
\hline \multirow{2}{*}{ PAT 6} & $* * *$ & *** & *** & *** & *** & *** & \\
\hline & 226.21 & 71.76 & 252.66 & 132.98 & 146.72 & 48.39 & -1.00 \\
\hline
\end{tabular}

c) Radiation Therapy (1984-2000) : Cumulative Survival Rate

\begin{tabular}{|c|c|c|c|c|c|c|c|c|}
\hline PATTERN & \multicolumn{2}{|c|}{ NO. OF CASES $* 1$} & $* 2$ & 1-YEAR & 2-YEAR & 3-YEAR & 4-YEAR & 5-YEAR \\
\hline & & 9 & & 9 & $\%$ & $\%$ & $\%$ & $\%$ \\
\hline PAT 0 none & 2,230 & 38.5 & 71 & 91.7 & 87.5 & 84.8 & 83.1 & 82.0 \\
\hline PAT 1 irradiated & 3,567 & 61.5 & 9 & 88.0 & 76.0 & 69.0 & 65.0 & 60.4 \\
\hline total & 5,797 & 100.0 & 80 & & & & & \\
\hline
\end{tabular}

*1 Number of cases who lived more than 30 days.

$* 2$ Number of cases who died within 30 days.

Mantel Chi-Square Test : Significant by $p<0.001$ 
d) Clinical Grading (1984-2000) : Cumulative Survival Rate

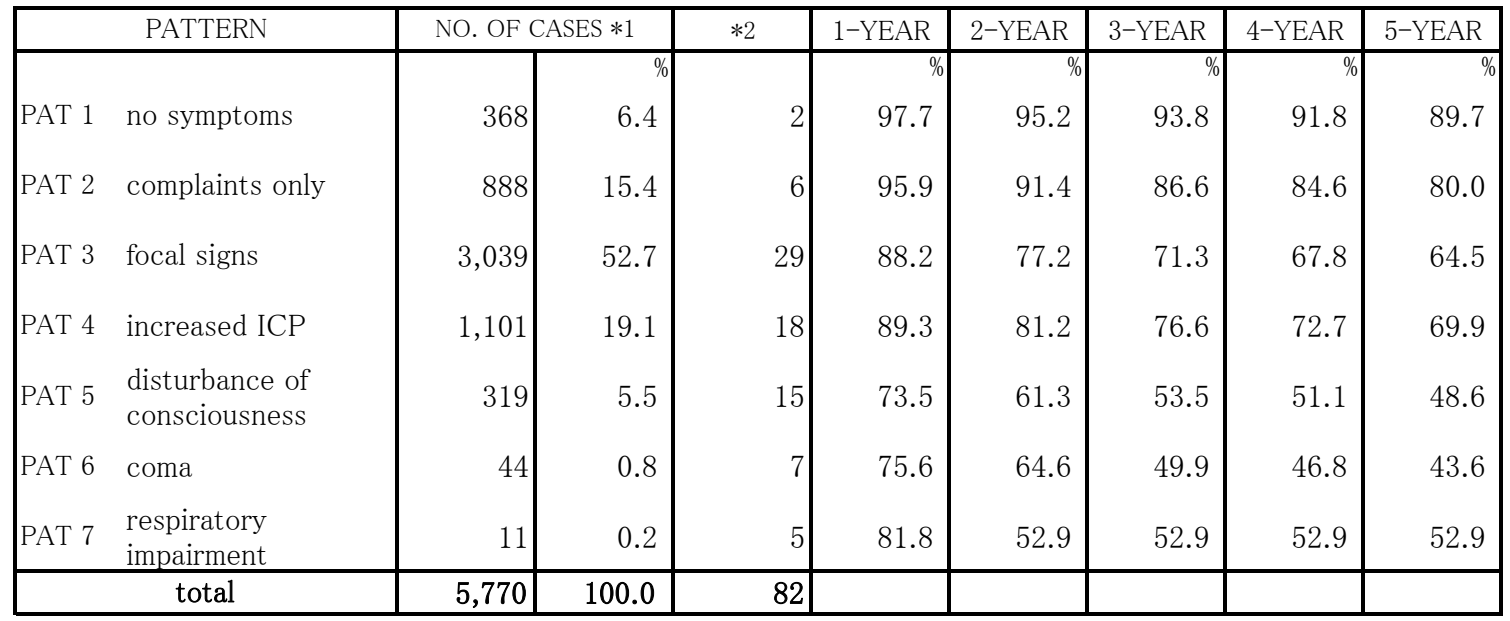

*1 Number of cases who lived more than 30 days.

$* 2$ Number of cases who died within 30 days.

Mantel Chi-Square Test

\begin{tabular}{|c|c|c|c|c|c|c|c|}
\hline & PAT 0 & PAT 1 & PAT 2 & PAT 3 & PAT 4 & PAT 5 & PAT 6 \\
\hline \multirow{2}{*}{ PAT 0} & & $* * *$ & $* * *$ & $* * *$ & $* * *$ & $* * *$ & $* * *$ \\
\hline & -1.00 & 12.08 & 65.76 & 43.81 & 107.48 & 60.69 & 17.09 \\
\hline \multirow{2}{*}{ PAT 1} & *** & & $* * *$ & $* * *$ & *** & $* * *$ & \\
\hline & 12.08 & -1.00 & 62.98 & 25.72 & 110.15 & 33.99 & 7.21 \\
\hline \multirow{2}{*}{ PAT 2} & *** & *** & & $* *$ & $* * *$ & & \\
\hline & 65.76 & 62.98 & -1.00 & 7.30 & 30.18 & 6.61 & 0.80 \\
\hline \multirow{2}{*}{ PAT 3} & *** & $* * *$ & $* *$ & & $* * *$ & $* * *$ & \\
\hline & 43.81 & 25.72 & 7.30 & -1.00 & 42.06 & 10.95 & 1.67 \\
\hline \multirow{2}{*}{ PAT 4} & *** & $* * *$ & $* * *$ & $* * *$ & & & \\
\hline & 107.48 & 110.15 & 30.18 & 42.06 & -1.00 & 0.09 & 0.03 \\
\hline \multirow{2}{*}{ PAT 5} & $* * *$ & $* * *$ & & $* * *$ & & & \\
\hline & 60.69 & 33.99 & 6.61 & 10.95 & 0.09 & -1.00 & 0.07 \\
\hline PAT 6 & $\begin{array}{r}* * * \\
17.09\end{array}$ & $\begin{array}{r}* * \\
7.21\end{array}$ & 0.80 & 1.67 & 0.03 & 0.07 & -1.00 \\
\hline
\end{tabular}

\section{1) Astrocytoma}

\section{Supratentorial Astrocytoma}

a-1) Operation (1984-2000): Cumulative Survival Rate

\begin{tabular}{|c|c|c|c|c|c|c|c|c|}
\hline PATTERN & NO. OF C & $* 1$ & $* 2$ & 1-YEAR & 2-YEAR & 3-YEAR & 4-YEAR & 5-YEAR \\
\hline & & $\%$ & & $\%$ & $\%$ & $\%$ & $\%$ & $\%$ \\
\hline PAT 0 none & 157 & 4.8 & 2 & 87.6 & 74.4 & 63.1 & 56.8 & 54.3 \\
\hline PAT 1 decompression & 41 & 1.2 & 3 & 78.7 & 68.8 & 57.7 & 53.8 & 53.8 \\
\hline biopsy or partial & 1,178 & 35.7 & 15 & 83.4 & 70.9 & 64.0 & 59.8 & 56.9 \\
\hline $50 \%$ resected & 311 & 9.4 & 6 & 85.9 & 76.3 & 70.2 & 65.0 & 61.1 \\
\hline PAT $475 \%$ resected & 574 & 17.4 & 7 & 88.4 & 75.5 & 66.7 & 63.1 & 56.4 \\
\hline $95 \%$ resected & 609 & 18.5 & 5 & 94.4 & 85.4 & 81.6 & 77.7 & 72.6 \\
\hline total removal & 427 & 13.0 & 4 & 97.8 & 94.9 & 91.2 & 89.2 & 87.8 \\
\hline total & 3,297 & 100.0 & 42 & & & & & \\
\hline
\end{tabular}

*1 Number of cases who lived more than 30 days.

*2 Number of cases who died within 30 days. 
Mantel Chi-Square Test

\begin{tabular}{|c|c|c|c|c|c|c|c|}
\hline & PAT 1 & PAT 2 & PAT 3 & PAT 4 & PAT 5 & PAT 6 & PAT 7 \\
\hline PAT 1 & -1.00 & 0.16 & 0.00 & 0.91 & 0.28 & $\begin{array}{r}* * * \\
15.80\end{array}$ & $\begin{array}{r}* * * \\
59.78\end{array}$ \\
\hline PAT 2 & 0.16 & -1.00 & 0.18 & 0.84 & 0.51 & $\begin{array}{r}\text { ** } \\
7.80\end{array}$ & $\begin{array}{r}\text { **** } \\
32.32\end{array}$ \\
\hline PAT 3 & 0.00 & 0.18 & -1.00 & 1.65 & 0.33 & $\begin{array}{r}\text { **** } \\
36.40\end{array}$ & $\begin{array}{r}* * * \\
93.99\end{array}$ \\
\hline PAT 4 & 0.91 & 0.84 & 1.65 & -1.00 & 0.60 & $\begin{array}{r}\text { **** } \\
12.07 \\
\end{array}$ & $\begin{array}{r}* * * * \\
57.06 \\
\end{array}$ \\
\hline PAT 5 & 0.28 & 0.51 & 0.33 & 0.60 & -1.00 & $\begin{array}{r}* * * * \\
25.34\end{array}$ & $\begin{array}{r}\text { **** } \\
81.41\end{array}$ \\
\hline PAT 6 & $\begin{array}{r}\text { *** } \\
15.80 \\
\end{array}$ & $\begin{array}{r}* * \\
7.80 \\
\end{array}$ & $\begin{array}{r}* * * \\
36.40 \\
\end{array}$ & $\begin{array}{r}* * * * \\
12.07 \\
\end{array}$ & $\begin{array}{r}\text { **** } \\
25.34 \\
\end{array}$ & -1.00 & $\begin{array}{r}\text { *** } \\
24.96\end{array}$ \\
\hline PAT 7 & $\begin{array}{r}* * * \\
59.78\end{array}$ & $\begin{array}{r}* * * \\
32.32\end{array}$ & $\begin{array}{r}* * * \\
93.99\end{array}$ & $\begin{array}{r}* * * \\
57.06\end{array}$ & $\begin{array}{r}\text { *** } \\
81.41\end{array}$ & $\begin{array}{r}* * * \\
24.96 \\
\end{array}$ & -1.00 \\
\hline
\end{tabular}

Infratentorial Astrocytoma

a-2) Operation (1984-2000) : Cumulative Survival Rate

\begin{tabular}{|ll|r|r|r|r|r|r|r|r|}
\hline \multicolumn{1}{|c|}{ PATTERN } & \multicolumn{2}{|c|}{ NO. OF CASES $* 1$} & $* 2$ & 1-YEAR & 2-YEAR & 3-YEAR & 4 -YEAR & 5-YEAR \\
\hline PAT 0 & none & 95 & 11.9 & 1 & 61.8 & 43.4 & 37.3 & 35.4 & 33.2 \\
PAT 1 decompression & 31 & 3.9 & 1 & 82.8 & 62.6 & 62.6 & 62.6 & 62.6 \\
PAT 2 & biopsy or partial & 183 & 23.0 & 4 & 76.1 & 57.1 & 50.6 & 50.6 & 46.6 \\
PAT 3 & $50 \%$ resected & 54 & 6.8 & 0 & 84.6 & 72.2 & 70.0 & 67.6 & 67.6 \\
PAT 4 75\% resected & 109 & 13.7 & 4 & 91.6 & 85.6 & 81.4 & 80.2 & 76.4 \\
PAT 5 95\% resected & 143 & 18.0 & 3 & 92.7 & 0.9 & 86.3 & 83.5 & 83.5 \\
PAT 6 total removal & 180 & 22.6 & 0 & 98.8 & 97.6 & 96.2 & 96.2 & 96.2 \\
\hline \multicolumn{1}{c}{ total } & 795 & 100.0 & 13 & & & & & \\
\hline
\end{tabular}

*1 Number of cases who lived more than 30 days.

$* 2$ Number of cases who died within 30 days.

\section{Mantel Chi-Square Test}

\begin{tabular}{|c|c|c|c|c|c|c|c|}
\hline & PAT 0 & PAT 1 & PAT 2 & PAT 3 & PAT 4 & PAT 5 & PAT 6 \\
\hline \multirow{2}{*}{ PAT 0} & & & & ** & *** & *** & *** \\
\hline & -1.00 & 3.87 & 3.26 & 8.88 & 27.71 & 42.12 & 93.59 \\
\hline \multirow{2}{*}{ PAT 1} & & & & & & * & $* * *$ \\
\hline & 3.87 & -1.00 & 1.19 & 0.14 & 2.34 & 5.47 & 31.37 \\
\hline \multirow{2}{*}{ PAT 2} & & & & & *** & *** & *** \\
\hline & 3.26 & 1.19 & -1.00 & 3.92 & 17.53 & 30.69 & 76.91 \\
\hline \multirow{2}{*}{ PAT 3} & $* *$ & & & & & * & *** \\
\hline & 8.88 & 0.14 & 3.92 & -1.00 & 1.54 & 4.89 & 30.81 \\
\hline \multirow{2}{*}{ PAT 4} & *** & & *** & & & & *** \\
\hline & 27.71 & 2.34 & 17.53 & 1.54 & -1.00 & 1.24 & 20.84 \\
\hline \multirow{2}{*}{ PAT 5} & *** & & *** & $*$ & & & *** \\
\hline & 42.12 & 5.47 & 30.69 & 4.89 & 1.24 & -1.00 & 12.42 \\
\hline \multirow{2}{*}{ PAT 6} & *** & *** & *** & $* * *$ & *** & $* * *$ & \\
\hline & 93.59 & 31.37 & 76.91 & 30.81 & 20.84 & 12.42 & -1.00 \\
\hline
\end{tabular}




\section{Supratentorial Astrocytoma}

b-1) Clinical Grading (1984-2000) : Cumulative Survival Rate

\begin{tabular}{|c|c|c|c|c|c|c|c|c|c|}
\hline & PATTERN & NO. OF C & ES $* 1$ & $* 2$ & 1-YEAR & 2-YEAR & 3-YEAR & 4-YEAR & 5-YEAR \\
\hline & & & $\%$ & & $\%$ & $\%$ & $\%$ & $\%$ & $\%$ \\
\hline PAT 1 & no symptoms & 230 & 6.9 & 1 & 96.8 & 93.3 & 91.6 & 88.6 & 85.2 \\
\hline PAT 2 & complaints only & 536 & 16.1 & 6 & 95.7 & 90.0 & 83.7 & 82.0 & 75.2 \\
\hline PAT 3 & focal signs & 1,917 & 57.7 & 20 & 88.2 & 76.2 & 70.1 & 65.6 & 62.0 \\
\hline PAT 4 & increased ICP & 419 & 12.6 & 4 & 85.1 & 73.7 & 66.0 & 60.0 & 56.4 \\
\hline PAT 5 & $\begin{array}{l}\text { disturbance of } \\
\text { concsiousness }\end{array}$ & 197 & 5.9 & 9 & 69.4 & 56.9 & 46.9 & 44.5 & 42.8 \\
\hline PAT 6 & coma & 21 & 0.6 & 2 & 73.7 & 49.1 & 36.8 & 36.8 & 36.8 \\
\hline PAT 7 & $\begin{array}{l}\text { respiratory } \\
\text { impairment }\end{array}$ & 1 & 0.0 & 1 & 100.0 & 0.0 & - & - & - \\
\hline & total & 3,321 & 100.0 & 43 & & & & & \\
\hline
\end{tabular}

*1 Number of cases who lived more than 30 days.

$* 2$ Number of cases who died within 30 days.

\section{Mantel Chi-Square Test}

\begin{tabular}{|c|c|c|c|c|c|c|c|}
\hline & PAT 1 & PAT 2 & PAT 3 & PAT 4 & PAT 5 & PAT 6 & PAT 7 \\
\hline \multirow{2}{*}{ PAT 1} & & & *** & $* * *$ & *** & $* * *$ & \\
\hline & -1.00 & 6.25 & 34.07 & 41.07 & 66.69 & 30.29 & 7.53 \\
\hline \multirow{2}{*}{ PAT 2} & & & *** & *** & $* * *$ & $* * *$ & \\
\hline & 6.25 & -1.00 & 28.78 & 35.30 & 71.64 & 18.83 & 4.74 \\
\hline \multirow{2}{*}{ PAT 3} & $* * *$ & $* * *$ & & & $* * *$ & & \\
\hline & 34.07 & 28.78 & -1.00 & 3.25 & 28.74 & 4.98 & 1.40 \\
\hline \multirow{2}{*}{ PAT 4} & $* * *$ & *** & & & & & \\
\hline & 41.07 & 35.30 & 3.25 & -1.00 & 10.09 & 2.77 & 1.23 \\
\hline \multirow{2}{*}{ PAT 5} & $* * *$ & $* * *$ & $* * *$ & $* *$ & & & \\
\hline & 66.69 & 71.64 & 28.74 & 10.09 & -1.00 & 0.09 & 0.46 \\
\hline \multirow{2}{*}{ PAT 6} & $* * *$ & $* * *$ & $*$ & & & & \\
\hline & 30.29 & 18.83 & 4.98 & 2.77 & 0.09 & -1.00 & 0.11 \\
\hline PAT 7 & $\begin{array}{r}* * * \\
7.53\end{array}$ & $\begin{array}{r}* * \\
4.74\end{array}$ & 1.40 & 1.23 & 0.46 & 0.11 & -1.00 \\
\hline
\end{tabular}


Infratentorial Astrocytoma

b-2) Clinical rading (1984-2000) : Cumulative Survival Rate

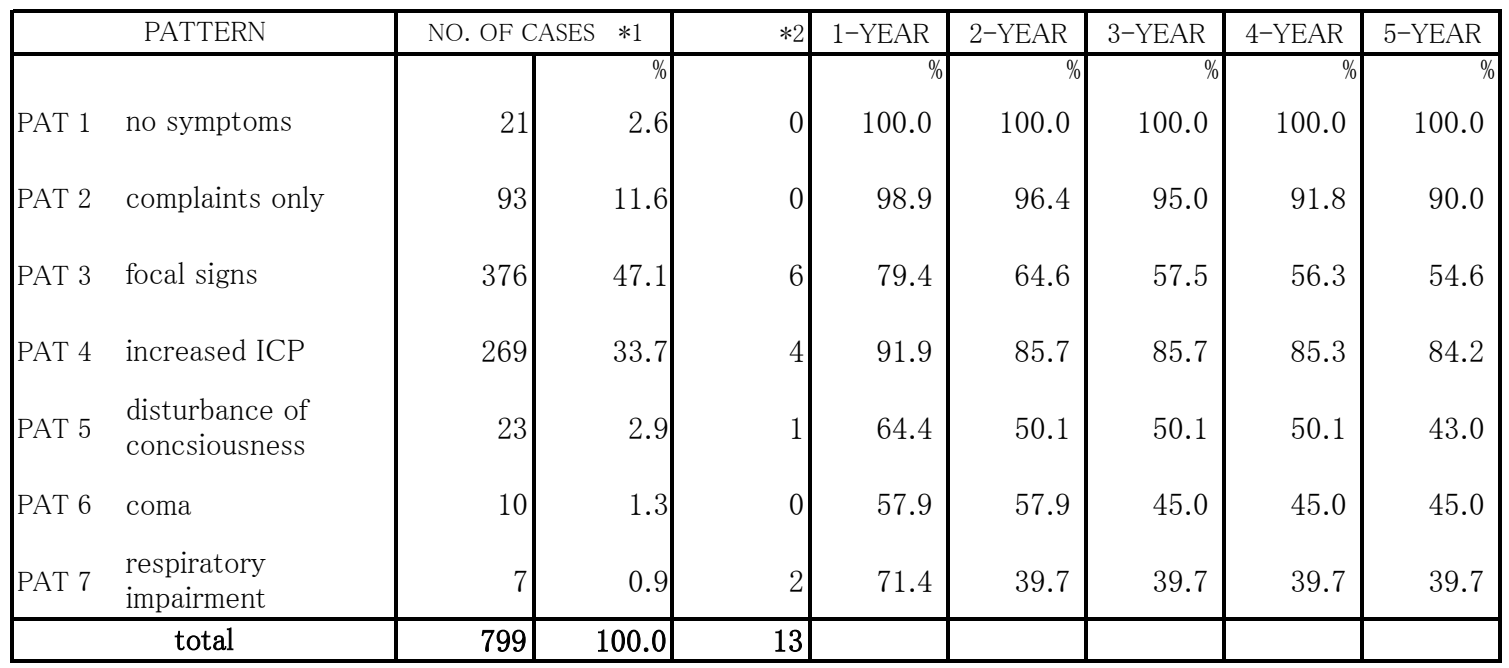

*1 Number of cases who lived more than 30 days.

$* 2$ Number of cases who died within 30 days.

Mantel Chi-Square Test

\begin{tabular}{|c|c|c|c|c|c|c|c|}
\hline & PAT 1 & PAT 2 & PAT 3 & PAT 4 & PAT 5 & PAT 6 & PAT 7 \\
\hline \multirow{2}{*}{ PAT 1} & & & ** & & $* * *$ & $* *$ & $* *$ \\
\hline & -1.00 & 1.49 & 9.17 & 2.89 & 10.63 & 8.80 & 9.78 \\
\hline \multirow{2}{*}{ PAT 2} & & & $* * *$ & & $* * *$ & $* * *$ & $* * *$ \\
\hline & 1.49 & -1.00 & 27.52 & 2.38 & 24.47 & 16.75 & 17.64 \\
\hline \multirow{2}{*}{ PAT 3} & $* *$ & $* * *$ & & $* * *$ & & & \\
\hline & 9.17 & 27.52 & -1.00 & 41.92 & 0.99 & 0.43 & 0.54 \\
\hline \multirow{2}{*}{ PAT 4} & & & $* * *$ & & $* * *$ & ** & \\
\hline & 2.89 & 2.38 & 41.92 & -1.00 & 17.29 & 8.78 & 7.28 \\
\hline \multirow{2}{*}{ PAT 5} & *** & *** & & $* * *$ & & & \\
\hline & 10.63 & 24.47 & 0.99 & 17.29 & -1.00 & 0.00 & 0.01 \\
\hline \multirow{2}{*}{ PAT 6} & ** & $* * *$ & & ** & & & \\
\hline & 8.80 & 16.75 & 0.43 & 8.78 & 0.00 & -1.00 & 0.02 \\
\hline \multirow{2}{*}{ PAT 7} & $* *$ & *** & & $* *$ & & & \\
\hline & 9.78 & 17.64 & 0.54 & 7.28 & 0.01 & 0.02 & -1.00 \\
\hline
\end{tabular}




\section{Supratentorial Astrocytoma}

c-1) Extension (1984-2000): Cumulative Survival Rate

\begin{tabular}{|c|c|c|c|c|c|c|c|c|}
\hline PATTERN & NO. OF C & ES $* 1$ & $* 2$ & 1-YEAR & 2-YEAR & 3-YEAR & 4-YEAR & 5-YEAR \\
\hline PAT 1 one side & 2,182 & 66.9 & 18 & 91.9 & 82.7 & 76.5 & 72.8 & 68.2 \\
\hline PAT 2 ventricular system & 367 & 11.2 & 9 & 80.4 & 68.3 & 61.5 & 57.3 & 55.2 \\
\hline PAT 3 central & 325 & 10.0 & 8 & 82.3 & 70.7 & 63.6 & 58.0 & 54.1 \\
\hline PAT 4 oppoite side & 220 & 6.7 & 6 & 76.6 & 58.2 & 49.1 & 43.1 & 40.2 \\
\hline $\begin{array}{ll}\text { PAT } 5 & \begin{array}{l}\text { supra }+ \\
\text { infratentorial }\end{array}\end{array}$ & 9 & 0.3 & 0 & 88.2 & 47.5 & 15.8 & 15.8 & \\
\hline PAT 6 other & 160 & 4.9 & 1 & 88.2 & 78.2 & 74.4 & 70.9 & 69.1 \\
\hline total & 3,263 & 100.0 & 42 & & & & & \\
\hline
\end{tabular}

$* 1$ Number of cases who lived more than 30 days.

$* 2$ Number of cases who died within 30 days.

Mantel Chi-Square Test

\begin{tabular}{|c|c|c|c|c|c|c|}
\hline & PAT 1 & PAT 2 & PAT 3 & PAT 4 & PAT 5 & PAT 6 \\
\hline \multirow{2}{*}{ PAT 1} & & *** & *** & $* * *$ & ** & \\
\hline & -1.00 & 26.70 & 23.59 & 63.08 & 9.07 & 0.05 \\
\hline \multirow{2}{*}{ PAT 2} & *** & & & * & & \\
\hline & 26.70 & -1.00 & 0.00 & 6.41 & 2.47 & 6.10 \\
\hline \multirow{2}{*}{ PAT 3} & *** & & & ** & & \\
\hline & 23.59 & 0.00 & -1.00 & 6.64 & 2.94 & 6.04 \\
\hline \multirow{2}{*}{ PAT 4} & *** & * & ** & & & *** \\
\hline & 63.08 & 6.41 & 6.64 & -1.00 & 0.68 & 18.52 \\
\hline PAT 5 & $\begin{array}{rr}* * \\
\end{array}$ & 247 & 294 & 068 & -100 & * \\
\hline \multirow[b]{2}{*}{ PAT 6} & 0.01 & * & $\frac{2.94}{*}$ & * $*$ ** & 1.00 & 0.14 \\
\hline & 0.05 & 6.10 & 6.04 & 18.52 & 6.14 & -1.00 \\
\hline
\end{tabular}

Infratentorial Astrocytoma

c-2) Extension (1984-2000) : Cumulative Survival Rate

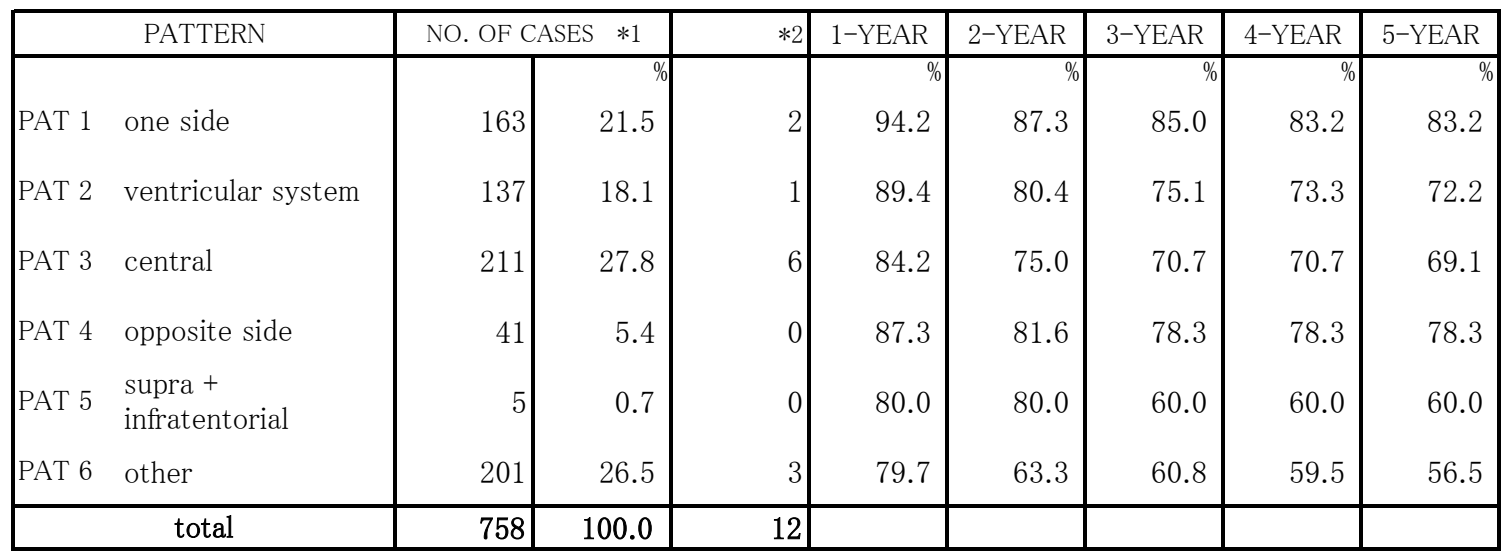

*1 Number of cases who lived more than 30 days.

$* 2$ Number of cases who died within 30 days. 
Mantel Chi-Square Test

\begin{tabular}{|c|c|c|c|c|c|c|}
\hline & PAT 1 & PAT 2 & PAT 3 & PAT 4 & PAT 5 & PAT 6 \\
\hline PAT 1 & -1.00 & $\begin{array}{r}* \\
3.99\end{array}$ & $\begin{array}{r}* * \\
7.76\end{array}$ & 0.64 & 1.73 & $\begin{array}{r}\text { **** } \\
20.97\end{array}$ \\
\hline PAT 2 & $\begin{array}{r}* \\
3.99 \\
\end{array}$ & -1.00 & 0.43 & 0.26 & 0.30 & $\begin{array}{r}* \\
6.03 \\
\end{array}$ \\
\hline PAT 3 & $\begin{array}{r}* * \\
7.76 \\
\end{array}$ & 0.43 & -1.00 & 0.85 & 0.14 & $\begin{array}{r}* \\
4.17 \\
\end{array}$ \\
\hline PAT 4 & 0.64 & 0.26 & 0.85 & -1.00 & 0.55 & 3.72 \\
\hline PAT 5 & 1.73 & 0.30 & 0.14 & 0.55 & -1.00 & 0.00 \\
\hline PAT 6 & $\begin{array}{r}* * * * \\
20.97\end{array}$ & $\begin{array}{r}* \\
6.03\end{array}$ & \begin{tabular}{r|}
$*$ \\
4.17 \\
\end{tabular} & 3.72 & 0.00 & -1.00 \\
\hline
\end{tabular}

Supratentorial Astrocytoma

d-1) Oparation With or Without Radiation Therapy (1984-2000) : Cumulative Survival Rate

\begin{tabular}{|c|c|c|c|c|c|c|c|c|c|}
\hline & PATTERN & NO. OF C & ES $* 1$ & $* 2$ & 1-YEAR & 2-YEAR & 3-YEAR & 4 4-YEAR & 5-YEAR \\
\hline & & & $\%$ & & $\%$ & $\%$ & $\%$ & $\%$ & $\%$ \\
\hline PAT 1 & $\begin{array}{l}\text { ope (biopsy) + } \\
\text { radiation }\end{array}$ & 892 & 28.9 & 1 & 83.4 & 69.0 & 61.1 & 56.3 & 52.7 \\
\hline PAT 2 & ope (biopsy) alone & 281 & 9.1 & 13 & 82.9 & 77.2 & 73.9 & 71.8 & 71.2 \\
\hline PAT 3 & $\begin{array}{l}\text { ope }(50-75 \% \\
\text { resected })+ \text { radiation }\end{array}$ & 668 & 21.7 & 0 & 88.7 & 75.3 & 66.7 & 62.1 & 56.1 \\
\hline PAT 4 & $\begin{array}{l}\text { ope }(50-75 \% \\
\text { resected) alone }\end{array}$ & 212 & 6.9 & 12 & 83.7 & 77.0 & 71.3 & 68.4 & 64.8 \\
\hline PAT 5 & $\begin{array}{l}\text { ope }(\geqq 95 \% \text { resected }) \\
+ \text { radiation }\end{array}$ & 586 & 19.0 & 3 & 95.2 & 85.5 & 79.9 & 75.6 & 69.9 \\
\hline PAT 6 & $\begin{array}{l}\text { ope }(\geqq 95 \% \text { resected }) \\
\text { alone }\end{array}$ & 446 & 14.5 & 5 & 96.7 & 94.3 & 92.9 & 91.6 & 90.6 \\
\hline PAT 7 & $\begin{array}{l}\text { ope (all cases) + } \\
\text { radiation }\end{array}$ & 2,171 & 69.4 & 4 & 88.1 & 75.3 & 67.7 & 63.1 & 58.2 \\
\hline PAT 8 & ope alone (all cases) & 955 & 30.6 & 33 & 89.6 & 85.3 & 82.2 & 80.4 & 78.9 \\
\hline
\end{tabular}

*1 Number of cases who lived more than 30 days.

$* 2$ Number of cases who died within 30 days.

Mantel Chi-Square Test

\begin{tabular}{|c|c|c|c|c|c|c|c|c|}
\hline & PAT 1 & PAT 2 & PAT 3 & PAT 4 & PAT 5 & PAT 6 & PAT 7 & PAT 8 \\
\hline \multirow{2}{*}{ PAT 1} & & *** & & & *** & **** & $* *$ & $* * *$ \\
\hline & -1.00 & 13.55 & 2.73 & 5.25 & 38.77 & 126.19 & 7.87 & 89.68 \\
\hline \multirow{2}{*}{ PAT 2} & *** & & & & & *** & $*$ & $*$ \\
\hline & 13.55 & -1.00 & 6.59 & 0.92 & 0.77 & 38.75 & 5.73 & 6.33 \\
\hline \multirow[t]{2}{*}{ PAT 3} & & & & & *** & *** & & $* * *$ \\
\hline & 2.73 & 6.59 & -1.00 & 1.59 & 21.01 & 102.00 & 0.34 & $\frac{56.98}{* * *}$ \\
\hline PAT 4 & $5.25^{*}$ & 0.92 & 1.59 & -1.00 & $\begin{array}{r}* \\
3.97\end{array}$ & $\begin{array}{r}\text { *** } \\
51.57\end{array}$ & 1.03 & $\begin{array}{r}* * * \\
12.93\end{array}$ \\
\hline \multirow{2}{*}{ PAT 5} & $* * *$ & & *** & & & *** & *** & $*$ \\
\hline & 38.77 & 0.77 & 21.01 & 3.97 & -1.00 & 42.20 & 22.70 & 5.46 \\
\hline \multirow{2}{*}{ PAT 6} & *** & *** & *** & $* * *$ & $* * *$ & & *** & **** \\
\hline & 126.19 & 38.75 & 102.00 & 51.57 & 42.20 & -1.00 & 105.66 & 23.36 \\
\hline \multirow[t]{2}{*}{ PAT 7} & ** & & & & **** & *** & & $* * *$ \\
\hline & $\begin{array}{r}7.87 \\
* * *\end{array}$ & $\frac{5.73}{*}$ & $\frac{0.34}{* * *}$ & $\frac{1.03}{* * *}$ & $\frac{22.70}{*}$ & $\frac{105.66}{* * *}$ & $\frac{-1.00}{* * *}$ & 69.24 \\
\hline PAT 8 & 89.68 & 6.33 & 56.98 & 12.93 & 5.46 & 23.36 & 69.24 & -1.00 \\
\hline
\end{tabular}


Infratentorial Astrocytoma

d-2) Oparation With or Without Radiation Therapy (1984-2000) : Cumulative Survival Rate

\begin{tabular}{|c|c|c|c|c|c|c|c|c|c|}
\hline & PATTERN & NO. OF C & $\mathrm{ES} * 1$ & $* 2$ & 1-YEAR & 2-YEAR & 3-YEAR & 4-YEAR & 5-YEAR \\
\hline & & & $\%$ & & $\%$ & $\%$ & $\%$ & $\%$ & $\%$ \\
\hline PAT 1 & $\begin{array}{l}\text { ope (biopsy) + } \\
\text { radiation }\end{array}$ & 133 & 20.0 & 2 & 76.4 & 55.4 & 46.6 & 46.6 & 41.2 \\
\hline PAT 2 & ope (biopsy) alone & 49 & 7.4 & 2 & 74.7 & 62.9 & 62.9 & 62.9 & 62.9 \\
\hline PAT 3 & $\begin{array}{l}\text { ope }(50-75 \% \\
\text { resected) }+\end{array}$ & 94 & 14.1 & 0 & 91.3 & 79.7 & 73.6 & 71.0 & 66.6 \\
\hline PAT 4 & $\begin{array}{l}\text { ope }(50-75 \% \\
\text { resected) alone }\end{array}$ & 69 & 10.4 & 4 & 86.7 & 83.5 & 83.5 & 83.5 & 83.5 \\
\hline PAT 5 & $\begin{array}{l}\text { ope }(\geqq 95 \% \\
\text { resected })+\end{array}$ & 68 & 10.2 & 0 & 92.4 & 85.8 & 84.0 & 80.1 & 80.1 \\
\hline PAT 6 & $\begin{array}{l}\text { ope }(\geqq 95 \% \\
\text { resected) alone }\end{array}$ & 253 & 38.0 & 3 & 97.1 & 95.3 & 93.8 & 93.3 & 93.3 \\
\hline PAT 7 & $\begin{array}{l}\text { ope (all cases) + } \\
\text { radiation }\end{array}$ & 315 & 45.2 & 2 & 84.2 & 69.1 & 63.2 & 61.5 & 58.1 \\
\hline PAT 8 & ope alone (all cases) & 382 & 54.8 & 10 & 92.4 & 88.8 & 87.8 & 87.4 & 87.4 \\
\hline
\end{tabular}

$* 1$ Number of cases who lived more than 30 days.

$* 2$ Number of cases who died within 30 days.

\section{Mantel Chi-Square Test}

\begin{tabular}{|c|c|c|c|c|c|c|c|c|}
\hline & PAT 1 & PAT 2 & PAT 3 & PAT 4 & PAT 5 & PAT 6 & PAT 7 & PAT 8 \\
\hline \multirow{2}{*}{ PAT 1} & & & & $* * *$ & $* * *$ & *** & $*$ & *** \\
\hline & -1.00 & 2.02 & 10.22 & 17.59 & 16.51 & 92.29 & 6.61 & 75.23 \\
\hline \multirow{2}{*}{ PAT 2} & & & & & & *** & & $* * *$ \\
\hline & 2.02 & -1.00 & 0.83 & 4.30 & 3.81 & 32.14 & 0.00 & 15.98 \\
\hline \multirow{2}{*}{ PAT 3} & & & & & & *** & & *** \\
\hline & 10.22 & 0.83 & -1.00 & 3.08 & 2.25 & 30.89 & 2.00 & 15.61 \\
\hline \multirow{2}{*}{ PAT 4} & *** & & & & & & $* *$ & \\
\hline & 17.59 & 4.30 & 3.08 & -1.00 & 0.08 & 6.32 & 8.73 & 0.78 \\
\hline \multirow{2}{*}{ PAT 5} & $* * *$ & & & & & ** & ** & \\
\hline & 16.51 & 3.81 & 2.25 & 0.08 & -1.00 & 8.70 & 7.46 & 1.68 \\
\hline \multirow{2}{*}{ PAT 6} & *** & *** & *** & & ** & & *** & $*$ \\
\hline & 92.29 & 32.14 & 30.89 & 6.32 & 8.70 & -1.00 & 64.10 & 5.17 \\
\hline \multirow{2}{*}{ PAT 7} & * & & & ** & ** & *** & & $* * *$ \\
\hline & 6.61 & 0.00 & 2.00 & 8.73 & 7.46 & 64.10 & -1.00 & 51.49 \\
\hline \multirow[t]{2}{*}{ PAT 8} & $\begin{array}{r}* * * \\
\end{array}$ & $\begin{array}{r}* * * \\
\end{array}$ & *** & & & * & *** & \\
\hline & 75.23 & 15.98 & 15.61 & 0.78 & 1.68 & 5.17 & 51.49 & -1.00 \\
\hline
\end{tabular}

e) Supra or Infratentorial Astrocytoma (1984-2000): Cumulative Survival Rate

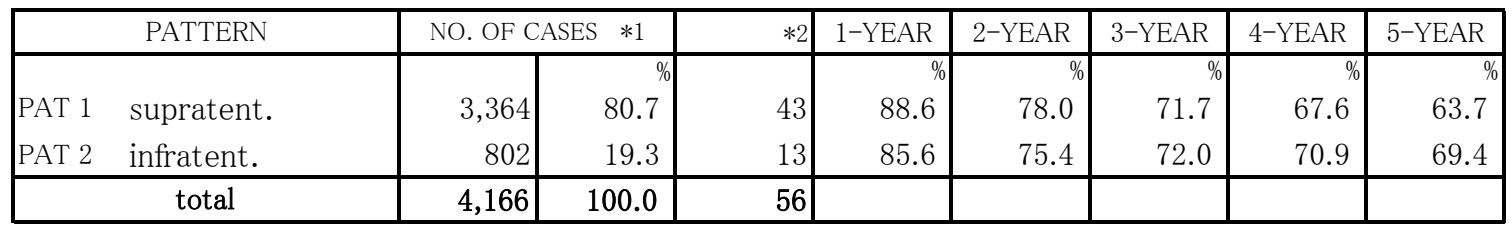

*1 Number of cases who lived more than 30 days.

$* 2$ Number of cases who died within 30 days.

Mantel Chi-Square Test : No Significancy Detected between Two of PATs 
12) Anaplastic Astrocytoma

a-1) Operation (1984-2000):

Cumulative Survival Rate of Supratentorial Anaplastic astrocytoma

\begin{tabular}{|ll|r|r|r|r|r|r|r|r|}
\hline & PATTERN & NO. OF CASES & $* 1$ & $* 2$ & 1-YEAR & 2-YEAR & 3-YEAR & 4-YEAR & 5-YEAR \\
\hline PAT 0 & none & 112 & 4.6 & 3 & 55.6 & 34.7 & 28.5 & 19.2 & 17.8 \\
PAT 1 & decompression & 33 & 1.4 & 1 & 62.5 & 32.9 & 16.4 & 9.9 & 6.6 \\
PAT 2 biopsy or partial & 761 & 31.2 & 22 & 59.0 & 32.5 & 23.5 & 18.4 & 16.2 \\
PAT 3 50\% resected & 273 & 11.2 & 10 & 60.8 & 35.9 & 25.5 & 17.1 & 15.7 \\
PAT 4 & 75\% resected & 603 & 24.7 & 15 & 71.6 & 46.8 & 35.7 & 29.1 & 24.7 \\
PAT 5 & 95\%resected & 478 & 19.6 & 3 & 81.9 & 60.4 & 48.3 & 41.6 & 35.3 \\
PAT 6 total removal & 182 & 7.5 & 0 & 84.1 & 64.8 & 57.1 & 51.7 & 50.1 \\
\hline \multicolumn{2}{c}{ total } & 2,442 & 100.0 & 54 & & & & & \\
\hline
\end{tabular}

*1 Number of cases who lived more than 30 days.

$* 2$ Number of cases who died within 30 days.

Mantel Chi-Square Test

\begin{tabular}{|c|c|c|c|c|c|c|c|}
\hline & PAT 0 & PAT 1 & PAT 2 & PAT 3 & PAT 4 & PAT 5 & PAT 6 \\
\hline PAT 0 & -1.00 & 0.41 & 0.01 & 0.00 & 3.29 & $\begin{array}{r}* * * \\
15.44\end{array}$ & $\begin{array}{r}\text { *** } \\
20.43\end{array}$ \\
\hline PAT 1 & 0.41 & -1.00 & 0.34 & 0.42 & 3.24 & $\begin{array}{r}* * * \\
10.47 \\
\end{array}$ & $\begin{array}{r}* * * \\
15.68 \\
\end{array}$ \\
\hline PAT 2 & 0.01 & 0.34 & -1.00 & 0.06 & $\begin{array}{r}* * * * \\
12.92 \\
\end{array}$ & $\begin{array}{r}* * * \\
46.06 \\
\end{array}$ & $\begin{array}{r}* * * * \\
42.05 \\
\end{array}$ \\
\hline PAT 3 & 0.00 & 0.42 & 0.06 & -1.00 & $\begin{array}{r}* \\
6.42 \\
\end{array}$ & $\begin{array}{r}* * * * \\
28.28 \\
\end{array}$ & $\begin{array}{r}* * * \\
31.23 \\
\end{array}$ \\
\hline PAT 4 & 3.29 & 3.24 & $\begin{array}{r}* * * * \\
12.92 \\
\end{array}$ & $\begin{array}{r}* \\
6.42 \\
\end{array}$ & -1.00 & $\begin{array}{r}* * * \\
11.91 \\
\end{array}$ & $\begin{array}{r}* * * * \\
19.64 \\
\end{array}$ \\
\hline PAT 5 & $\begin{array}{r}* * * \\
15.44 \\
\end{array}$ & $\begin{array}{r}* * * * \\
10.47\end{array}$ & $\begin{array}{r}* * * \\
46.06 \\
\end{array}$ & $\begin{array}{r}* * * \\
28.28 \\
\end{array}$ & $\begin{array}{r}* * * \\
11.91 \\
\end{array}$ & -1.00 & 4.48 \\
\hline PAT 6 & $\begin{array}{r}* * * * \\
20.43 \\
\end{array}$ & $\begin{array}{r}* * * * \\
15.68 \\
\end{array}$ & $\begin{array}{r}* * * * \\
42.05 \\
\end{array}$ & $\begin{array}{r}* * * \\
31.23 \\
\end{array}$ & $\begin{array}{r}* * * * \\
19.64 \\
\end{array}$ & $\begin{array}{r}* * \\
4.48 \\
\end{array}$ & -1.00 \\
\hline
\end{tabular}


a-2) Operation (1984-2000):

Cumulative Survival Rate of Infratentorial Anaplastic astrocytoma

\begin{tabular}{|ll|r|r|r|r|r|r|r|r|}
\hline & PATTERN & \multicolumn{2}{|c|}{ NO. OF CASES $* 1$} & $* 2$ & 1-YEAR & 2-YEAR & 3-YEAR & 4-YEAR & 5-YEAR \\
\hline PAT 0 & none & 51 & 19.1 & 5 & 43.1 & 13.7 & 8.2 & 8.2 & 8.2 \\
PAT 1 & decompression & 6 & 2.2 & 0 & 50.0 & 33.3 & 33.3 & 16.7 & 16.7 \\
PAT 2 biopsy or partial & 99 & 37.1 & 2 & 51.1 & 32.8 & 29.0 & 25.1 & 19.3 \\
PAT 3 50\% resected & 18 & 6.7 & 1 & 52.9 & 24.7 & 24.7 & 16.5 & 16.5 \\
PAT 4 & 75\% resected & 45 & 16.9 & 1 & 72.4 & 56.3 & 34.4 & 31.1 & 27.5 \\
PAT 5 & 95\%resected & 38 & 14.2 & 1 & 83.1 & 70.1 & 52.1 & 48.2 & 39.8 \\
PAT 6 total removal & 10 & 3.7 & 0 & 100.0 & 100.0 & 100.0 & 100.0 & 100.0 \\
\hline \multicolumn{1}{r|}{ total } & 267 & 100.0 & 10 & & & & & \\
\hline
\end{tabular}

*1 Number of cases who lived more than 30 days.

$* 2$ Number of cases who died within 30 days.

\section{Mantel Chi-Square Test}

\begin{tabular}{|c|c|c|c|c|c|c|c|}
\hline & PAT 0 & PAT 1 & PAT 2 & PAT 3 & PAT 4 & PAT 5 & PAT 6 \\
\hline PAT 0 & -1.00 & 0.19 & 1.18 & 0.21 & $\begin{array}{r}* \\
4.89 \\
\end{array}$ & $\begin{array}{r}* * \\
9.12 \\
\end{array}$ & $\begin{array}{r}* * \\
9.44 \\
\end{array}$ \\
\hline PAT 1 & 0.19 & -1.00 & 0.00 & 0.01 & 0.35 & 1.42 & 6.04 \\
\hline PAT 2 & 1.18 & 0.00 & -1.00 & 0.07 & 1.17 & $\begin{array}{r}* * \\
4.05 \\
\end{array}$ & $\begin{array}{r}* * \\
8.32 \\
\end{array}$ \\
\hline PAT 3 & 0.21 & 0.01 & 0.07 & -1.00 & 1.35 & 3.80 & $\begin{array}{r}* * \\
7.10 \\
\end{array}$ \\
\hline PAT 4 & $\begin{array}{r}* \\
4.89 \\
\end{array}$ & 0.35 & 1.17 & 1.35 & -1.00 & 1.09 & $\begin{array}{r}* \\
6.59 \\
\end{array}$ \\
\hline PAT 5 & $\begin{array}{r}* * \\
9.12 \\
\end{array}$ & 1.42 & $\begin{array}{r}* * \\
4.05 \\
\end{array}$ & 3.80 & 1.09 & -1.00 & $\begin{array}{r}* \\
4.99 \\
\end{array}$ \\
\hline PAT 6 & $\begin{array}{r}* * * \\
9.44\end{array}$ & $\begin{array}{r}* \\
6.04\end{array}$ & $\begin{array}{r}* * * \\
8.32\end{array}$ & $\begin{array}{r}* * \\
7.10 \\
\end{array}$ & $\begin{array}{r}* * \\
6.59\end{array}$ & $\begin{array}{r}* * \\
4.99\end{array}$ & -1.00 \\
\hline
\end{tabular}

\section{b-1) Clinical Grading (1984-2000) : \\ Cumulative Survival Rate of Supratentorial Anaplastic Astrocytoma}

\begin{tabular}{|c|c|c|c|c|c|c|c|c|c|}
\hline & PATTERN & NO. OF & S $* 1$ & $* 2$ & 1-YEAR & 2-YEAR & 3-YEAR & 4-YEAR & 5-YEAR \\
\hline & & & $\%$ & & $\%$ & $\%$ & $\%$ & $\%$ & $\%$ \\
\hline PAT 1 & no symptoms & 42 & 1.7 & 0 & 90.1 & 79.7 & 68.9 & 57.1 & 53.9 \\
\hline PAT 2 & coplaints only & 245 & 10.0 & 1 & 86.4 & 64.5 & 53.8 & 45.0 & 38.1 \\
\hline PAT 3 & focal signs & 1,481 & 60.5 & 21 & 68.6 & 44.4 & 33.1 & 27.5 & 24.0 \\
\hline PAT 4 & increased ICP & 411 & 16.8 & 9 & 66.2 & 40.7 & 30.3 & 23.9 & 21.1 \\
\hline PAT 5 & $\begin{array}{l}\text { disturbace of } \\
\text { concsiousness }\end{array}$ & 245 & 10.0 & 16 & 49.8 & 23.4 & 20.5 & 14.9 & 14.3 \\
\hline PAT 6 & coma & 21 & 0.9 & 6 & 65.0 & 53.2 & 53.2 & 33.2 & 33.2 \\
\hline PAT 7 & $\begin{array}{l}\text { respiratory } \\
\text { impairment }\end{array}$ & 3 & 0.1 & 0 & 60.0 & 60.0 & 60.0 & 60.0 & 60.0 \\
\hline & total & 2,448 & 100.0 & 53 & & & & & \\
\hline
\end{tabular}

*1 Number of cases who lived more than 30 days.

$* 2$ Number of cases who died within 30 days. 
Mantel Chi-Square Test

\begin{tabular}{|c|c|c|c|c|c|c|c|}
\hline & PAT 1 & PAT 2 & PAT 3 & PAT 4 & PAT 5 & PAT 6 & PAT 7 \\
\hline PAT 1 & -1.00 & 2.25 & $\begin{array}{r}* * * \\
10.29\end{array}$ & $\begin{array}{r}\text { *** } \\
11.79\end{array}$ & $\begin{array}{r}* * * \\
16.14\end{array}$ & 2.16 & 0.08 \\
\hline PAT 2 & 2.25 & -1.00 & $\begin{array}{r}* * * \\
18.38 \\
\end{array}$ & $\begin{array}{r}* * * * \\
20.10\end{array}$ & $\begin{array}{r}* * * \\
36.55 \\
\end{array}$ & 0.79 & 0.00 \\
\hline PAT 3 & $\begin{array}{r}* * * \\
10.29 \\
\end{array}$ & $\begin{array}{r}* * * * \\
18.38 \\
\end{array}$ & -1.00 & 0.91 & $\begin{array}{r}* * * * \\
13.81 \\
\end{array}$ & 0.25 & 0.26 \\
\hline PAT 4 & $\begin{array}{r}* * * * \\
11.79 \\
\end{array}$ & $\begin{array}{r}* * * \\
20.10 \\
\end{array}$ & 0.91 & -1.00 & $\begin{array}{r}* \\
5.76 \\
\end{array}$ & 0.57 & 0.33 \\
\hline PAT 5 & $\begin{array}{r}* * * \\
16.14 \\
\end{array}$ & $\begin{array}{r}* * * \\
36.55 \\
\end{array}$ & $\begin{array}{r}* * * \\
13.81 \\
\end{array}$ & $\begin{array}{r}* \\
5.76 \\
\end{array}$ & -1.00 & 2.01 & 0.58 \\
\hline PAT 6 & 2.16 & 0.79 & 0.25 & 0.57 & 2.01 & -1.00 & 0.10 \\
\hline PAT 7 & 0.08 & 0.00 & 0.26 & 0.33 & 0.58 & 0.10 & -1.00 \\
\hline
\end{tabular}

b-2) Clinical Grading(1984-2000):

Cumulative Survival Rate of Infratentorial Anaplastic Astrocytoma

\begin{tabular}{|c|c|c|c|c|c|c|c|c|c|}
\hline & PATTERN & NO. OF C & $* 1$ & $* 2$ & 1-YEAR & 2-YEAR & 3-YEAR & 4-YEAR & 5-YEAR \\
\hline & & & $\%$ & & $\%$ & $\%$ & $\%$ & $\%$ & $\%$ \\
\hline PAT 1 & no symptoms & 3 & 1.1 & 0 & 100.0 & 100.0 & 100.0 & 66.7 & 33.3 \\
\hline PAT 2 & complaints only & 14 & 5.2 & 0 & 76.9 & 76.9 & 41.4 & 41.4 & 41.4 \\
\hline PAT 3 & focal signs & 183 & 68.0 & 5 & 58.8 & 38.3 & 30.5 & 27.6 & 23.6 \\
\hline PAT 4 & increased ICP & 47 & 17.5 & 3 & 75.0 & 44.0 & 35.7 & 30.0 & 26.7 \\
\hline PAT 5 & $\begin{array}{l}\text { disturbance of } \\
\text { consiousness }\end{array}$ & 17 & 6.3 & 1 & 16.1 & 0.0 & - & - & - \\
\hline PAT 6 & coma & 3 & 1.1 & 1 & 0.0 & - & - & - & - \\
\hline PAT 7 & $\begin{array}{l}\text { respiratory } \\
\text { impairment }\end{array}$ & 2 & 0.7 & 0 & 0.0 & - & - & - & - \\
\hline & total & 269 & 100.0 & 10 & & & & & \\
\hline
\end{tabular}

*1 Number of cases who lived more than 30 days.

$* 2$ Number of cases who died within 30 days.

Mantel Chi-Square Test

\begin{tabular}{|l|r|r|r|r|r|r|r|}
\hline & \multicolumn{1}{|c|}{ PAT 1 } & \multicolumn{1}{c|}{ PAT 2} & \multicolumn{1}{c|}{ PAT 3 } & PAT 4 & PAT 5 & PAT 6 & PAT 7 \\
\hline PAT 1 & -1.00 & 0.05 & 0.38 & 0.39 & 3.67 & 2.00 & 1.80 \\
\hline PAT 2 & 0.05 & -1.00 & 1.34 & 0.59 & 4.90 & 2.05 & 1.58 \\
\hline PAT 3 & 0.38 & 1.34 & -1.00 & 0.44 & 3.73 & 1.21 & 0.82 \\
\hline PAT 4 & 0.39 & 0.59 & 0.44 & -1.00 & 6.29 & 2.71 & 1.92 \\
\hline PAT 5 & 3.67 & 4.90 & 3.73 & 6.29 & -1.00 & 0.04 & 0.03 \\
\hline PAT 6 & 2.00 & 2.05 & 1.21 & 2.71 & 0.04 & -1.00 & 0.00 \\
\hline PAT 7 & 1.80 & 1.58 & 0.82 & 1.92 & 0.03 & 0.00 & -1.00 \\
\hline
\end{tabular}


c) Extension (1984-2000):

Cumulative Survival Rate of Supratentorial Anaplastic Astrocytoma

\begin{tabular}{|c|c|c|c|c|c|c|c|c|}
\hline PATTERN & NO. OF C & ES $* 1$ & $* 2$ & 1-YEAR & 2-YEAR & 3-YEAR & 4-YEAR & 5-YEAR \\
\hline & & $\%$ & & $\%$ & $\%$ & $\%$ & $\%$ & $\%$ \\
\hline PAT 1 one side & 1,496 & 61.7 & 23 & 74.5 & 51.8 & 40.3 & 33.4 & 29.0 \\
\hline PAT 2 ventricular system & 332 & 13.7 & 10 & 58.4 & 30.8 & 21.9 & 17.4 & 16.9 \\
\hline PAT 3 central & 295 & 12.2 & 10 & 59.9 & 33.9 & 24.6 & 20.4 & 17.6 \\
\hline PAT 4 opposite side & 237 & 9.8 & 8 & 58.2 & 33.5 & 27.0 & 19.0 & 16.7 \\
\hline $\begin{array}{ll}\text { PAT } 5 & \begin{array}{l}\text { supra }+ \\
\text { infratentorial }\end{array}\end{array}$ & 8 & 0.3 & 0 & 60.0 & 45.0 & 15.0 & 0.0 & - \\
\hline other & 56 & 2.3 & 0 & 46.3 & 31.9 & 29.4 & 26.2 & 26.2 \\
\hline total & 2,424 & 100.0 & 51 & & & & & \\
\hline
\end{tabular}

*1 Number of cases who lived more than 30 days.

$* 2$ Number of cases who died within than 30 days.

\section{Mantel Chi-Square Test}

\begin{tabular}{|l|r|r|r|r|r|r|}
\hline & PAT 1 & PAT 2 & PAT 3 & PAT 4 & PAT 5 & PAT 6 \\
\hline PAT 1 & -1.00 & $\begin{array}{r}* * * \\
22.11\end{array}$ & $\begin{array}{r}* * * \\
16.47\end{array}$ & $\begin{array}{r}* * * \\
14.73\end{array}$ & 1.72 & 3.59 \\
\hline PAT 2 & $\begin{array}{r}* * * \\
22.11\end{array}$ & -1.00 & 0.10 & 0.03 & 0.15 & 0.04 \\
\hline PAT 3 & $\begin{array}{r}* * * \\
16.47\end{array}$ & 0.10 & -1.00 & 0.01 & 0.27 & 0.00 \\
\hline PAT 4 & $\begin{array}{r}* * * \\
14.73\end{array}$ & 0.03 & 0.01 & -1.00 & 0.27 & 0.02 \\
\hline PAT 5 & 1.72 & 0.15 & 0.27 & 0.27 & -1.00 & 0.34 \\
\hline PAT 6 & 3.59 & 0.04 & 0.00 & 0.02 & 0.34 & -1.00 \\
\hline
\end{tabular}


d-1) Operation With or Without Radiation Therapy (1984-2000): Cumulative Survival Rate of Supratentorial Anaplastic Astrocytoma

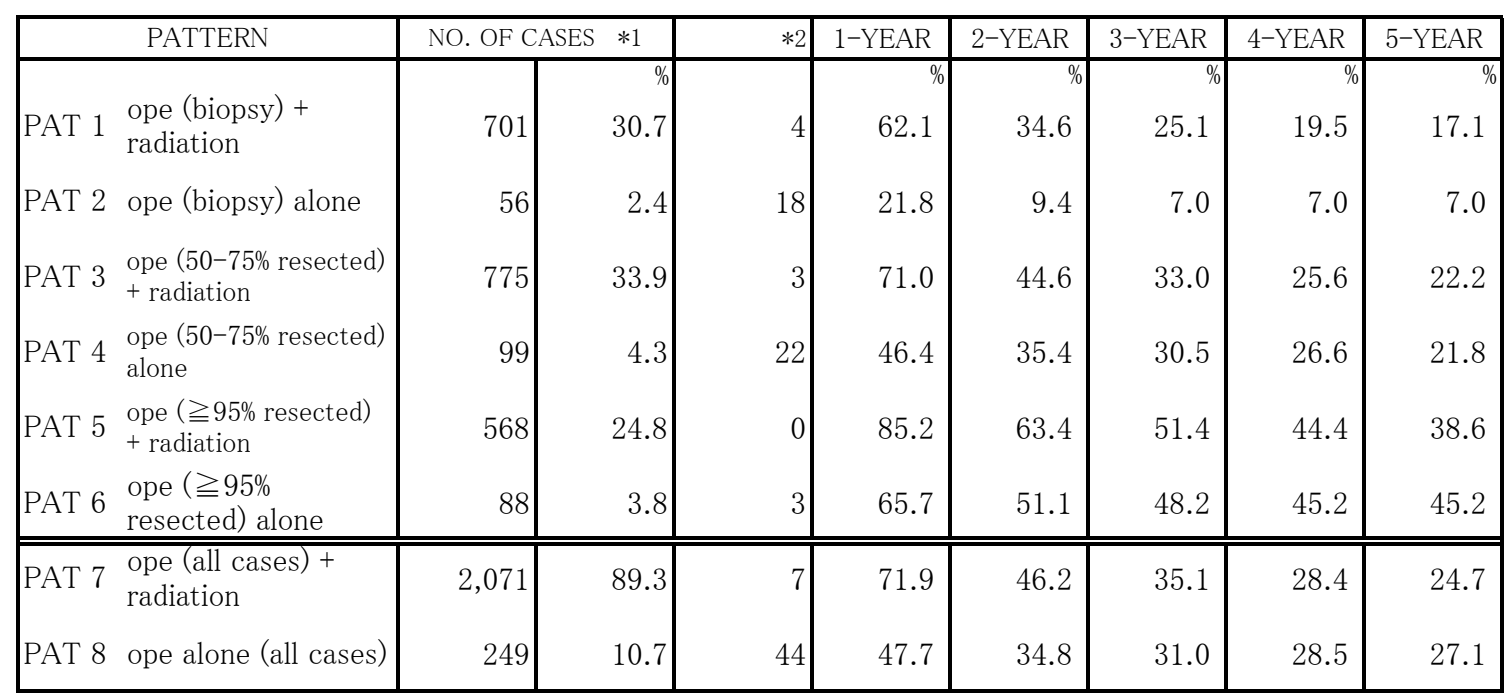

*1 Number of cases who lived more than 30 days.

$* 2$ Number of cases who died within 30 days.

\section{Mantel Chi-Square Test}

\begin{tabular}{|c|c|c|c|c|c|c|c|c|}
\hline & PAT 1 & PAT 2 & PAT 3 & PAT 4 & PAT 5 & PAT 6 & PAT 7 & PAT 8 \\
\hline \multirow{2}{*}{ PAT 1} & & *** & $* * *$ & & $* * *$ & $* * *$ & $* * *$ & \\
\hline & -1.00 & 26.95 & 13.20 & 0.25 & 105.44 & 16.24 & 29.38 & 0.11 \\
\hline \multirow{2}{*}{ PAT 2} & *** & & *** & *** & *** & $* * *$ & $* * *$ & $* * *$ \\
\hline & 26.95 & -1.00 & 46.33 & 11.06 & 105.58 & 31.31 & 53.96 & 15.74 \\
\hline \multirow{2}{*}{ PAT 3} & $* * *$ & *** & & & $* * *$ & $* *$ & & $*$ \\
\hline & 13.20 & 46.33 & -1.00 & 4.81 & 52.63 & 6.88 & 1.33 & 4.08 \\
\hline \multirow{2}{*}{ PAT 4} & & **** & $*$ & & $* * *$ & ** & ** & \\
\hline & 0.25 & 11.06 & 4.81 & -1.00 & 34.56 & 8.50 & 7.54 & 0.20 \\
\hline \multirow{2}{*}{ PAT 5} & *** & *** & *** & *** & & & $* * *$ & *** \\
\hline & 105.44 & 105.58 & 52.63 & 34.56 & -1.00 & 0.47 & 52.16 & 46.94 \\
\hline \multirow{2}{*}{ PAT 6} & $* * *$ & $* * *$ & $* * *$ & $* * *$ & $* * *$ & & & ** \\
\hline & 16.24 & 16.24 & 16.24 & 16.24 & 16.24 & -1.00 & 5.25 & 8.90 \\
\hline \multirow{2}{*}{ PAT 7} & $* * *$ & $* * *$ & & $* *$ & $* * *$ & $*$ & & $* *$ \\
\hline & 29.38 & 53.96 & 1.33 & 7.54 & 52.16 & 5.25 & -1.00 & 8.86 \\
\hline \multirow{2}{*}{ PAT 8} & & *** & $*$ & & $* * *$ & $* *$ & ** & \\
\hline & 0.11 & 15.74 & 4.08 & 0.20 & 46.94 & 8.90 & 8.86 & -1.00 \\
\hline
\end{tabular}


d-2) Operation With or Without Radiation Therapy (1984-2000): Cumulative Survival Rate of Infratentorial Anaplastic Astrocytoma

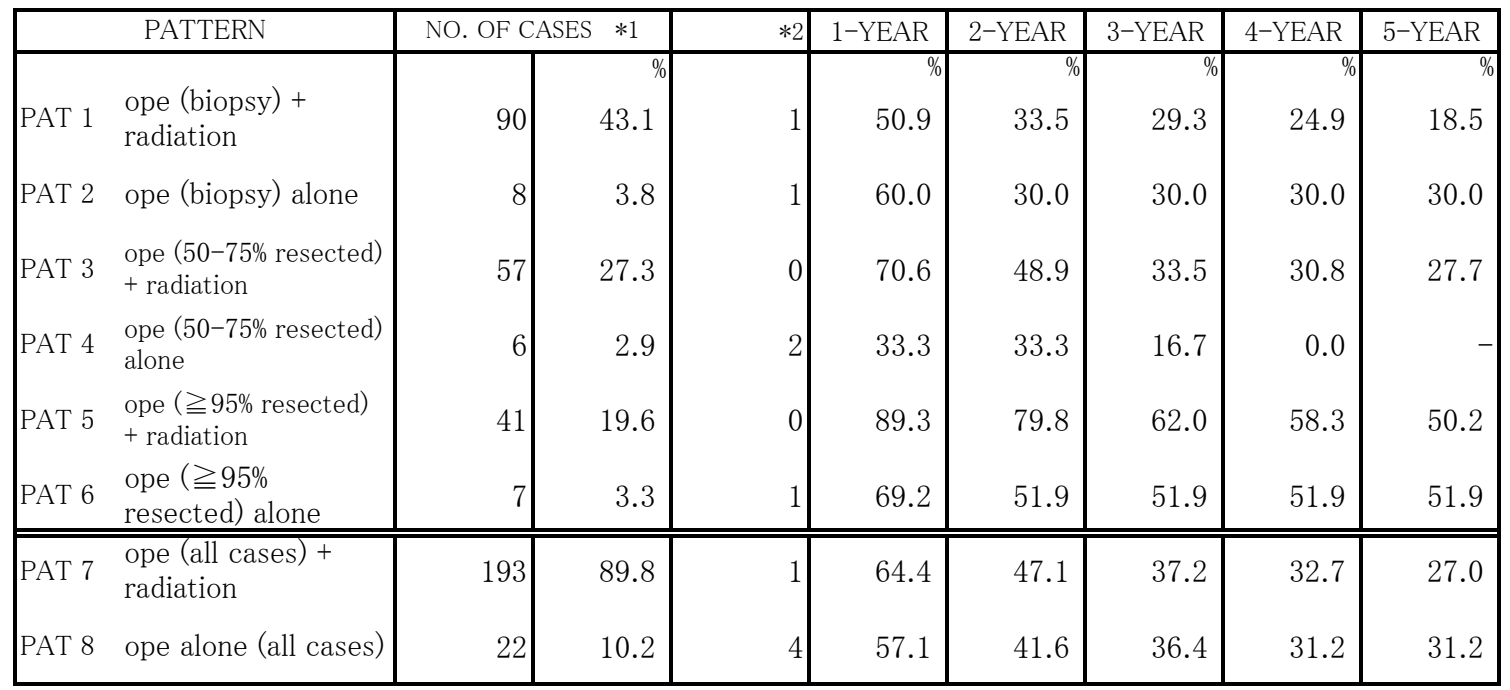

*1 Number of cases who lived more than 30 days.

$* 2$ Number of cases who died within 30 days.

\section{Mantel Chi-Square Test}

\begin{tabular}{|l|r|r|r|r|r|r|r|r|}
\hline & PAT 1 & \multicolumn{1}{|c|}{ PAT 2 } & \multicolumn{1}{c|}{ PAT 3 } & PAT 4 & PAT 5 & PAT 6 & PAT 7 & PAT 8 \\
\hline PAT 1 & -1.00 & 0.17 & 1.27 & 0.69 & 8.92 & 1.34 & 1.68 & 0.48 \\
\hline PAT 2 & 0.17 & -1.00 & 0.03 & 0.48 & 2.09 & 0.23 & 0.00 & 0.00 \\
\hline PAT 3 & 0.69 & 0.48 & -1.00 & 1.55 & 6.49 & 1.40 & 1.58 & 0.84 \\
\hline PAT 4 & 0.69 & 0.48 & 1.55 & -1.00 & 6.49 & 1.40 & 1.58 & 0.84 \\
\hline PAT 5 & 8.92 & 2.09 & 4.40 & 6.49 & -1.00 & 0.12 & 5.78 & 2.96 \\
\hline PAT 6 & 1.34 & 0.23 & 0.44 & 1.40 & 0.12 & -1.00 & 0.67 & 0.42 \\
\hline PAT 7 & 1.68 & 0.00 & 0.03 & 1.58 & 5.78 & 0.67 & -1.00 & 0.00 \\
\hline PAT 8 & 0.48 & 0.00 & 0.02 & 0.84 & 2.96 & 0.42 & 0.00 & -1.00 \\
\hline
\end{tabular}


e) Chemotherapy (1981-2000):

Cumulative Survival Rate of Supratentorial Anaplastic Astrocytoma

\begin{tabular}{|ll|r|r|r|r|r|r|r|r|}
\hline & PATTERN & \multicolumn{2}{|c|}{ NO. OF CASES $* 1$} & $* 2$ & 1-YEAR & 2-YEAR & 3-YEAR & 4 -YEAR & 5-YEAR \\
\hline PAT 1 & chemo(-) & 761 & 33.3 & 47 & 61.1 & 41.3 & 32.1 & 26.5 & 23.1 \\
PAT 2 & 5FU or Tegafur & 89 & 3.9 & 0 & 61.7 & 35.9 & 27.5 & 19.8 & 18.0 \\
PAT 3 ACNU & 1,168 & 51.1 & 3 & 73.8 & 47.7 & 37.4 & 30.7 & 27.3 \\
PAT 4 ACNU + VCR & 269 & 11.8 & 0 & 72.9 & 45.8 & 36.8 & 31.1 & 27.3 \\
\hline \multicolumn{1}{|c|}{ total } & 2,287 & 100.0 & 50 & & & & & \\
\hline
\end{tabular}

$* 1$ Number of cases who lived more than 30 days.

*2 Number of cases who died within 30 days.

Mantel Chi-Square Test : Significancy Detected Only between PAT1 and PAT3 by $p<0.01$

f) Supra or Infratentorial Anaplastic Astrocytoma (1984-2000) : Cumulative Survival Rate

\begin{tabular}{|c|c|c|c|c|c|c|c|c|}
\hline PATTERN & NO. OF C & $* 1$ & $* 2$ & 1-YEAR & 2-YEAR & 3-YEAR & 4-YEAR & 5-YEAR \\
\hline & & $\%$ & & $\%$ & $\%$ & $\%$ & $\%$ & $\%$ \\
\hline supratent. & 2,474 & 90 & 54 & 68.4 & 44.4 & 34.2 & 28.0 & 24.5 \\
\hline infratent. & 271 & 10 & 10 & 59.2 & 38.8 & 30.5 & 27.0 & 23.1 \\
\hline total & 2,745 & 100 & 64 & & & & & \\
\hline
\end{tabular}

*1 Number of cases who lived more than 30 days.

$* 2$ Number of caes who died within 30 days.

Mantel Chi-Square Test : No Significancy Detected between Two of PATs 
13) Medulloblastoma

a) Operation (1984-2000) : Cumulative Survival Rate

\begin{tabular}{|ll|r|r|r|r|r|r|r|r|}
\hline & PATTERN & \multicolumn{2}{|c|}{ NO. OF CASES $* 1$} & $* 2$ & 1-YEAR & 2-YEAR & 3-YEAR & 4 -YEAR & 5-YEAR \\
\hline PAT 0 & none & 24 & 3.7 & 1 & 73.9 & 69.3 & 69.3 & 64.2 & 58.8 \\
PAT 1 & decompression & 37 & 5.7 & 5 & 74.6 & 59.4 & 40.1 & 33.5 & 29.9 \\
PAT 2 & biopsy or partial & 46 & 7.1 & 2 & 69.2 & 62.3 & 57.4 & 57.4 & 49.2 \\
PAT 3 & 50\% resected & 35 & 5.4 & 0 & 85.1 & 65.8 & 62.5 & 59.2 & 55.7 \\
PAT 4 & 75\% resected & 101 & 15.7 & 4 & 81.3 & 63.5 & 55.3 & 45.1 & 38.2 \\
PAT 5 & 95\% resected & 238 & 36.9 & 3 & 84.1 & 68.2 & 60.2 & 51.1 & 44.5 \\
PAT 6 & total removal & 164 & 25.4 & 2 & 92.5 & 84.0 & 74.8 & 70.2 & 66.9 \\
\hline \multicolumn{2}{r|}{ total } & 645 & 100.0 & 17 & & & & & \\
\hline
\end{tabular}

$* 1$ Number of cases who lived more than 30 days.

$* 2$ Number of cases who died within 30 days.

Mantel Chi-Square Test

\begin{tabular}{|c|c|c|c|c|c|c|c|}
\hline & PAT 0 & PAT 1 & PAT 2 & PAT 3 & PAT 4 & PAT 5 & PAT 6 \\
\hline PAT 0 & -1.00 & 2.43 & 0.34 & 0.01 & 1.38 & 0.63 & 0.91 \\
\hline PAT 1 & 2.43 & -1.00 & 1.44 & 2.60 & 0.74 & 2.48 & $\begin{array}{r}* * * \\
14.69 \\
\end{array}$ \\
\hline PAT 2 & 0.34 & 1.44 & -1.00 & 0.27 & 0.42 & 0.00 & $\begin{array}{r}* \\
5.18 \\
\end{array}$ \\
\hline PAT 3 & 0.01 & 2.60 & 0.27 & -1.00 & 1.53 & 0.65 & 1.72 \\
\hline PAT 4 & 1.38 & 0.74 & 0.42 & 1.53 & -1.00 & 0.75 & $\begin{array}{r}* * * \\
15.10\end{array}$ \\
\hline PAT 5 & 0.63 & 2.48 & 0.00 & 0.65 & 0.75 & -1.00 & $\begin{array}{r}\text { *** } \\
13.34\end{array}$ \\
\hline PAT 6 & 0.91 & $\begin{array}{r}* * * \\
14.69 \\
\end{array}$ & $\begin{array}{r}* * \\
5.18 \\
\end{array}$ & 1.72 & $\begin{array}{r}* * * \\
15.10 \\
\end{array}$ & $\begin{array}{r}* * * \\
13.34 \\
\end{array}$ & -1.00 \\
\hline
\end{tabular}

b) Operation With or Without Radiation Therapy (1984-2000) : Cumulative Survival Rate

\begin{tabular}{|c|c|c|c|c|c|c|c|c|c|}
\hline & PATTERN & NO. OF C & S $* 1$ & $* 2$ & 1-YEAR & 2-YEAR & 3-YEAR & 4-YEAR & 5-YEAR \\
\hline & & & $\%$ & & $\%$ & $\%$ & $\%$ & $\%$ & $\%$ \\
\hline PAT 1 & $\begin{array}{l}\text { ope }(\text { biopsy) + } \\
\text { radiation }\end{array}$ & 44 & 7.6 & 1 & 70.1 & 62.9 & 57.7 & 57.7 & 49.1 \\
\hline PAT 2 & ope (biopsy) alone & 2 & 0.3 & 1 & 50.0 & 50.0 & 50.0 & 50.0 & 50.0 \\
\hline PAT 3 & $\begin{array}{l}\text { ope }(50-75 \% \text { resected }) \\
+ \text { radiation }\end{array}$ & 123 & 21.2 & 0 & 85.7 & 66.8 & 61.2 & 52.2 & 45.8 \\
\hline PAT 4 & $\begin{array}{l}\text { ope }(50-75 \% \text { resected }) \\
\text { alone }\end{array}$ & 11 & 1.9 & 4 & 40.0 & 40.0 & 13.3 & 13.3 & 13.3 \\
\hline PAT 5 & $\begin{array}{l}\text { ope }(\geqq 95 \% \text { resected }) \\
+ \text { radiation }\end{array}$ & 364 & 62.8 & 1 & 89.1 & 75.7 & 66.7 & 58.8 & 53.0 \\
\hline PAT 6 & $\begin{array}{l}\text { ope }(\geqq 95 \% \\
\text { resected) alone }\end{array}$ & 36 & 6.2 & 4 & 71.4 & 65.3 & 62.2 & 62.2 & 62.2 \\
\hline PAT 7 & $\begin{array}{l}\text { ope (all cases) + } \\
\text { radiation }\end{array}$ & 562 & 91.1 & 2 & 86.5 & 72.2 & 63.5 & 55.9 & 49.8 \\
\hline PAT 8 & ope alone (all cases) & 55 & 8.9 & 14 & 60.4 & 56.3 & 49.9 & 49.9 & 49.9 \\
\hline
\end{tabular}

*1 Number of cases who lived more than 30 days.

$* 2$ Number of cases who died within 30 days. 
Mantel Chi-Square Test

\begin{tabular}{|c|c|c|c|c|c|c|c|c|}
\hline & PAT 1 & PAT 2 & PAT 3 & PAT 4 & PAT 5 & PAT 6 & PAT 7 & PAT 8 \\
\hline PAT 1 & -1.00 & 0.00 & 0.00 & 2.54 & 0.84 & 0.55 & 0.27 & 0.01 \\
\hline PAT 2 & 0.00 & -1.00 & 0.02 & 0.23 & 0.11 & 0.06 & 0.05 & 0.00 \\
\hline PAT 3 & 0.00 & 0.02 & -1.00 & $\begin{array}{r}* * \\
6.70 \\
\end{array}$ & 1.55 & 0.59 & 0.44 & 0.16 \\
\hline PAT 4 & 2.54 & 0.23 & $\begin{array}{r}* * \\
6.70\end{array}$ & -1.00 & $\begin{array}{r}\text { *** } \\
10.79\end{array}$ & 3.13 & $\begin{array}{r}* * \\
8.73\end{array}$ & 1.52 \\
\hline PAT 5 & 0.84 & 0.11 & 1.55 & $\begin{array}{r}* * * \\
10.79 \\
\end{array}$ & -1.00 & 0.02 & 0.84 & 2.20 \\
\hline PAT 6 & 0.55 & 0.06 & 0.59 & 3.13 & 0.02 & -1.00 & 0.23 & 0.66 \\
\hline PAT 7 & 0.27 & 0.05 & 0.44 & $\begin{array}{r}* * \\
8.73 \\
\end{array}$ & 0.84 & 0.23 & -1.00 & 1.18 \\
\hline PAT 8 & 0.01 & 0.00 & 0.16 & 1.52 & 2.20 & 0.66 & 1.18 & -1.00 \\
\hline
\end{tabular}

c) Clinical Grading (1984-2000): Cumulative Survival Rate

\begin{tabular}{|c|c|c|c|c|c|c|c|c|c|}
\hline & PATTERN & NO. OF C & ES $* 1$ & $* 2$ & 1-YEAR & 2-YEAR & 3-YEAR & 4-YEAR & 5-YEAR \\
\hline & & & $\%$ & & $\%$ & $\%$ & $\%$ & $\%$ & $\%$ \\
\hline PAT 1 & no symptoms & 7 & 1.1 & 0 & 85.7 & 85.7 & 66.7 & 66.7 & 66.7 \\
\hline PAT 2 & complaints only & 53 & 8.3 & 0 & 89.9 & 89.9 & 82.7 & 69.6 & 63.7 \\
\hline PAT 3 & focal signs & 136 & 21.2 & 1 & 85.4 & 78.8 & 74.4 & 66.0 & 62.0 \\
\hline PAT 4 & increased ICP & 410 & 64.0 & 6 & 83.6 & 66.8 & 58.4 & 52.5 & 46.2 \\
\hline PAT 5 & $\begin{array}{l}\text { disturbance of } \\
\text { consciousness }\end{array}$ & 31 & 4.8 & 4 & 0.8 & 66.5 & 43.7 & 43.7 & 39.6 \\
\hline PAT 6 & coma & 4 & 0.6 & 3 & 25.0 & 0.0 & - & - & - \\
\hline PAT 7 & $\begin{array}{l}\text { respiratory } \\
\text { impairment }\end{array}$ & 0 & 0.0 & 2 & - & - & - & - & - \\
\hline & total & 641 & 100.0 & 16 & & & & & \\
\hline
\end{tabular}

*1 Number of cases who lived more than 30 days.

$* 2$ Number of cases who died within 30 days. 
Mantel Chi-Square Test

\begin{tabular}{|l|r|r|r|r|r|r|r|}
\hline & PAT 1 & PAT 2 & PAT 3 & PAT 4 & PAT 5 & PAT 6 & PAT 7 \\
\hline PAT 1 & -1.00 & 0.02 & 0.02 & 0.56 & 0.92 & 3.56 & -1.00 \\
\hline PAT 2 & 0.02 & -1.00 & 0.24 & 4.37 & 4.77 & 12.19 & -1.00 \\
\hline PAT 3 & 0.02 & 0.24 & -1.00 & 6.30 & 4.25 & 8.52 & -1.00 \\
\hline PAT 4 & 0.56 & 4.37 & 6.30 & -1.00 & 0.49 & 6.15 & -1.00 \\
\hline PAT 5 & 0.92 & 4.77 & 4.25 & 0.49 & -1.00 & 3.51 & -1.00 \\
\hline PAT 6 & 3.56 & 12.19 & 8.52 & 6.15 & 3.51 & -1.00 & -1.00 \\
\hline PAT 7 & -1.00 & -1.00 & -1.00 & -1.00 & -1.00 & -1.00 & -1.00 \\
\hline
\end{tabular}

d) Extension (1984-2000) : Cumulative Survival Rate

\begin{tabular}{|c|c|c|c|c|c|c|c|c|c|}
\hline & PATTERN & NO. OF & $\mathrm{ES} * 1$ & $* 2$ & 1-YEAR & 2-YEAR & 3-YEAR & 4-YEAR & 5-YEAR \\
\hline & & & $\%$ & & $\%$ & $\%$ & $\%$ & $\%$ & $\%$ \\
\hline PAT 1 & one side & 35 & 5.6 & 1 & 82.4 & 75.8 & 71.9 & 58.0 & 52.4 \\
\hline PAT 2 & ventricular system & 290 & 46.6 & 9 & 87.0 & 72.7 & 62.8 & 58.6 & 52.0 \\
\hline PAT 3 & central & 103 & 16.6 & 4 & 82.8 & 71.9 & 65.0 & 60.0 & 52.1 \\
\hline PAT 4 & opposite side & 13 & 2.1 & 0 & 84.6 & 84.6 & 76.9 & 44.5 & 35.6 \\
\hline PAT 5 & $\begin{array}{l}\text { supra }+ \\
\text { infratentorial }\end{array}$ & 18 & 2.9 & 0 & 61.1 & 44.4 & 26.7 & 26.7 & 20.0 \\
\hline PAT 6 & other & 163 & 26.2 & 3 & 81.5 & 64.4 & 57.8 & 49.9 & 48.2 \\
\hline & total & 622 & 100.0 & 17 & & & & & \\
\hline
\end{tabular}

*1 Number of cases who lived more than 30 days.

$* 2$ Number of cases who died within than 30 days.

\section{Mantel Chi-Square Test}

\begin{tabular}{|l|r|r|r|r|r|r|}
\hline & PAT 1 & PAT 2 & PAT 3 & PAT 4 & PAT 5 & PAT 6 \\
\hline PAT 1 & -1.00 & 0.00 & 0.02 & 0.18 & 4.50 & 0.39 \\
\hline PAT 2 & 0.00 & -1.00 & 0.01 & 0.51 & 7.44 & 1.04 \\
\hline PAT 3 & 0.02 & 0.01 & -1.00 & 0.36 & 5.70 & 0.44 \\
\hline PAT 4 & 0.18 & 0.51 & 0.36 & -1.00 & 2.13 & 0.06 \\
\hline PAT 5 & 4.50 & 7.44 & 5.70 & 2.13 & -1.00 & 4.42 \\
\hline PAT 6 & 0.39 & 1.04 & 0.44 & 0.06 & 4.42 & -1.00 \\
\hline
\end{tabular}


e) Chemotherapy (1984-2000): Cumulative Survival Rate

\begin{tabular}{|ll|r|r|r|r|r|r|r|r|}
\hline & PATTERN & NO. OF CASES $* 1$ & $* 2$ & 1-YEAR & 2-YEAR & 3-YEAR & 4-YEAR & 5-YEAR \\
\hline PAT 1 & chemo(-) & 217 & 45.8 & 16 & 79.0 & 67.4 & 60.9 & 54.4 & 48.7 \\
PAT 2 & 5FU or Tegafur & 8 & 1.7 & 0 & 86.7 & 43.3 & 43.3 & 28.9 & 28.9 \\
PAT 3 & MTX & 76 & 16.0 & 0 & 85.2 & 73.7 & 62.3 & 55.3 & 51.7 \\
PAT 4 & ACNU & 100 & 21.1 & 1 & 89.9 & 74.2 & 65.4 & 55.9 & 48.3 \\
PAT 5 & ACNU + VCR & 73 & 15.4 & 1 & 93.1 & 79.8 & 70.1 & 63.3 & 52.5 \\
\hline \multicolumn{1}{|c|}{ total } & 474 & 100.0 & 18 & & & & & \\
\hline
\end{tabular}

*1 Number of cases who lived more than 30 days.

$* 2$ Number of cases who died within 30 days.

Mantel Chi-Square Test : No Signuficancy Detected between Each of PATs

14) Pontine Glioma

a) Operation (1984-2000): Cumulative Survival Rate

\begin{tabular}{|c|c|c|c|c|c|c|c|c|c|}
\hline & PATTERN & NO. OF C & ES $* 1$ & $* 2$ & 1-YEAR & 2-YEAR & 3-YEAR & 4-YEAR & 5-YEAR \\
\hline & & & $\%$ & & $\%$ & $\%$ & $\%$ & $\%$ & $\%$ \\
\hline PAT 0 & none & 316 & 48.1 & 7 & 49.3 & 23.9 & 17.1 & 13.9 & 11.5 \\
\hline PAT 1 & decompression & 45 & 6.8 & 1 & 39.5 & 16.9 & 10.2 & 10.2 & 10.2 \\
\hline PAT 2 & biopsy or partial & 238 & 36.2 & 6 & 56.0 & 38.3 & 32.7 & 30.0 & 26.3 \\
\hline PAT 3 & $50 \%$ resected & 19 & 2.9 & 0 & 57.9 & 31.6 & 26.3 & 26.3 & 26.3 \\
\hline PAT 4 & $75 \%$ resected & 21 & 3.2 & 2 & 76.2 & 45.7 & 33.5 & 33.5 & 33.5 \\
\hline PAT 5 & $95 \%$ resected & 16 & 2.4 & 0 & 80.0 & 80.0 & 80.0 & 80.0 & 80.0 \\
\hline PAT 6 & total removal & 2 & 0.3 & 0 & 50.0 & 50.0 & 50.0 & 50.0 & 50.0 \\
\hline & total & 657 & 100.0 & 16 & & & & & \\
\hline
\end{tabular}

*1 Number of cases who lived more than 30 days.

$* 2$ Number of cases who died within 30 days.

\section{Mantel Chi-Square Test}

\begin{tabular}{|c|c|c|c|c|c|c|c|}
\hline & PAT 0 & PAT 1 & PAT 2 & PAT 3 & PAT 4 & PAT 5 & PAT 6 \\
\hline PAT 0 & -1.00 & 0.30 & $\begin{array}{r}* \\
5.94\end{array}$ & 0.95 & 2.95 & $\begin{array}{r}* * * \\
12.13 \\
\end{array}$ & 0.56 \\
\hline PAT 1 & 0.30 & -1.00 & 2.77 & 0.89 & 2.89 & $\begin{array}{r}* * \\
9.27 \\
\end{array}$ & 0.48 \\
\hline PAT 2 & $\begin{array}{r}* \\
5.94 \\
\end{array}$ & 2.77 & -1.00 & 0.00 & 0.41 & $\begin{array}{r}* * \\
7.22 \\
\end{array}$ & 0.20 \\
\hline PAT 3 & 0.95 & 0.89 & 0.00 & -1.00 & 0.36 & $\begin{array}{r}* \\
4.90 \\
\end{array}$ & 0.11 \\
\hline PAT 4 & 2.95 & 2.89 & 0.41 & 0.36 & -1.00 & 3.50 & 0.01 \\
\hline PAT 5 & $\begin{array}{r}* * * * \\
12.13 \\
\end{array}$ & $\begin{array}{r}* * * \\
9.27 \\
\end{array}$ & $\begin{array}{r}* * * \\
7.22 \\
\end{array}$ & $\begin{array}{r}* \\
4.90 \\
\end{array}$ & 3.50 & -1.00 & 0.44 \\
\hline PAT 6 & 0.56 & 0.48 & 0.20 & 0.11 & 0.01 & 0.44 & -1.00 \\
\hline
\end{tabular}


b) Radiation Therapy (1984-2000) : Cumulative Survival Rate

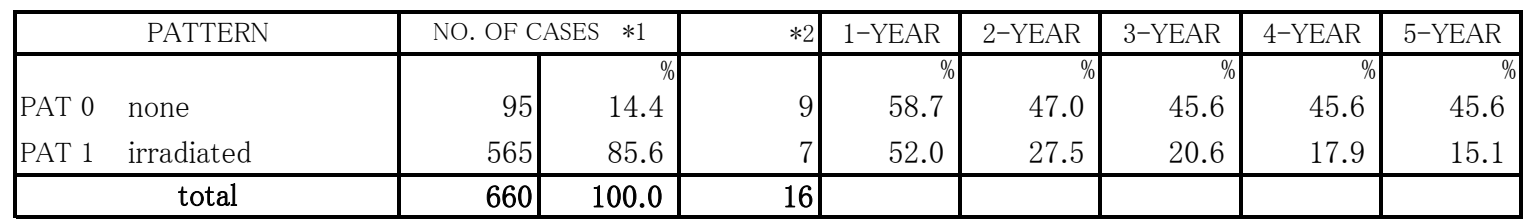

$* 1$ Number of cases who lived more than 30 days.

$* 2$ Number of cases who died within 30 days.

Mantel Chi-Square Test $*$ No Significancy Between Two of PATs

c) Clinical Grading (1984-2000) : Cumulative Survival Rate

\begin{tabular}{|c|c|c|c|c|c|c|c|c|c|}
\hline & PATTERN & NO. OF C & S $\quad * 1$ & $* 2$ & 1 -YEAR & $2-\mathrm{YEAR}$ & 3-YEAR & 4-YEAR & 5 -YEAR \\
\hline & & & $\%$ & & $\%$ & $\%$ & $\%$ & $\%$ & $\%$ \\
\hline PAT 1 & no symptoms & 8 & 1.2 & 0 & 60.0 & 60.0 & 60.0 & 40.0 & 40.0 \\
\hline PAT 2 & complaints only & 34 & 5.2 & 0 & 76.7 & 59.1 & 53.5 & 53.5 & 53.5 \\
\hline PAT 3 & focal signs & 498 & 75.8 & 10 & 55.3 & 31.7 & 24.4 & 22.0 & 19.4 \\
\hline PAT 4 & increased ICP & 60 & 9.1 & 2 & 48.3 & 21.8 & 19.5 & 19.5 & 19.5 \\
\hline PAT 5 & $\begin{array}{l}\text { disturbance of } \\
\text { consciousness }\end{array}$ & 34 & 5.2 & 0 & 24.2 & 9.3 & 9.3 & 9.3 & 9.3 \\
\hline PAT 6 & coma & 12 & 1.8 & 2 & 36.4 & 12.1 & 12.1 & 12.1 & 0.0 \\
\hline PAT 7 & $\begin{array}{l}\text { respiratory } \\
\text { impairment }\end{array}$ & 11 & 1.7 & 1 & 27.3 & 27.3 & 27.3 & 27.3 & 27.3 \\
\hline & total & 657 & 100.0 & 15 & & & & & \\
\hline
\end{tabular}

$* 1$ Number of cases who lived more than 30 days.

$* 2$ Number of cases who died within 30 days.

\section{Mantel Chi-Square Test}

\begin{tabular}{|l|r|r|r|r|r|r|r|}
\hline & PAT 1 & PAT 2 & PAT 3 & PAT 4 & PAT 5 & PAT 6 & PAT 7 \\
\hline PAT 1 & -1.00 & 0.19 & 0.52 & 0.48 & 1.50 & 1.36 & 0.18 \\
\hline PAT 2 & 0.19 & -1.00 & 5.25 & 4.52 & 6.88 & 4.58 & 1.86 \\
\hline PAT 3 & 0.52 & 5.25 & -1.00 & 0.20 & 3.35 & 1.09 & 0.08 \\
\hline PAT 4 & 0.48 & 4.52 & 0.20 & -1.00 & 1.02 & 0.55 & 0.02 \\
\hline PAT 5 & 1.50 & $\begin{array}{r}* * \\
*\end{array}$ & 3.35 & 1.02 & -1.00 & 0.01 & 0.21 \\
\hline PAT 6 & 1.36 & 4.58 & 1.09 & 0.55 & 0.01 & -1.00 & 0.22 \\
\hline PAT 7 & 0.18 & 1.86 & 0.08 & 0.02 & 0.21 & 0.22 & -1.00 \\
\hline
\end{tabular}


15) Ependymoma

a) Operation (1984-2000): Cumulative Survival Rate

\begin{tabular}{|ll|r|r|r|r|r|r|r|r|}
\hline & PATTERN & \multicolumn{2}{|c|}{ NO. OF CASES $* 1$} & $* 2$ & 1-YEAR & 2-YEAR & 3-YEAR & 4-YEAR & 5-YEAR \\
\hline PAT 0 & none & 16 & 3.3 & 3 & 69.2 & 69.2 & 23.1 & $\%$ & - \\
PAT 1 & decompression & 10 & 2.0 & 1 & 100.0 & 77.8 & 64.8 & 64.8 & 64.8 \\
PAT 2 & biopsy or partial & 39 & 8.0 & 0 & 77.5 & 70.3 & 62.0 & 57.7 & 53.3 \\
PAT 3 & $50 \%$ resected & 36 & 7.3 & 1 & 83.3 & 77.7 & 71.8 & 62.5 & 48.9 \\
PAT 4 & 75\% resected & 77 & 15.7 & 5 & 90.6 & 75.2 & 69.3 & 67.8 & 64.2 \\
PAT 5 & 95\% resected & 138 & 28.2 & 3 & 94.8 & 89.3 & 83.2 & 77.5 & 74.4 \\
PAT 6 & total removal & 174 & 35.5 & 0 & 98.8 & 94.3 & 90.7 & 88.4 & 84.1 \\
\hline \multicolumn{2}{r|}{ total } & 490 & 100.0 & 13 & & & & & \\
\hline
\end{tabular}

*1 Number of cases who lived more than 30 days.

*2 Number of cases who died within 30 days.

\section{Manatel Chi-Square Test}

\begin{tabular}{|c|c|c|c|c|c|c|c|}
\hline & PAT 0 & PAT 1 & PAT 2 & PAT 3 & PAT 4 & PAT 5 & PAT 6 \\
\hline PAT 0 & -1.00 & 1.42 & 0.74 & 2.08 & 3.58 & $\begin{array}{r}* * * * \\
11.01 \\
\end{array}$ & $\begin{array}{r}* * * \\
27.43 \\
\end{array}$ \\
\hline PAT 1 & 1.42 & -1.00 & 0.41 & 0.30 & 0.03 & 0.40 & 2.42 \\
\hline PAT 2 & 0.74 & 0.41 & -1.00 & 0.00 & 1.18 & $\begin{array}{r}* \\
6.30 \\
\end{array}$ & $\begin{array}{r}* * * \\
18.38 \\
\end{array}$ \\
\hline PAT 3 & 2.08 & 0.30 & 0.00 & -1.00 & 1.15 & $\begin{array}{r}* \\
6.28 \\
\end{array}$ & $\begin{array}{r}* * * \\
18.79 \\
\end{array}$ \\
\hline PAT 4 & 3.58 & 0.03 & 1.18 & 1.15 & -1.00 & 2.54 & $\begin{array}{r}* * * * \\
12.11 \\
\end{array}$ \\
\hline PAT 5 & $\begin{array}{r}* * * * \\
11.01\end{array}$ & 0.40 & $\begin{array}{r}* \\
6.30\end{array}$ & $\begin{array}{r}* \\
6.28 \\
\end{array}$ & 2.54 & -1.00 & $\begin{array}{r}* \\
4.08\end{array}$ \\
\hline PAT 6 & $\begin{array}{r}* * * * \\
27.43 \\
\end{array}$ & 2.42 & $\begin{array}{r}* * * * \\
18.38 \\
\end{array}$ & $\begin{array}{r}* * * \\
18.79 \\
\end{array}$ & $\begin{array}{r}* * * \\
12.11 \\
\end{array}$ & $\begin{array}{r}* \\
4.08 \\
\end{array}$ & -1.00 \\
\hline
\end{tabular}

\section{b) Clinical Grading (1984-2000): Cumulative Survival Rate}

\begin{tabular}{|c|c|c|c|c|c|c|c|c|c|}
\hline & PATTERN & NO. OF C & $\mathrm{ES} * 1$ & $* 2$ & 1-YEAR & 2-YEAR & 3-YEAR & 4-YEAR & 5-YEAR \\
\hline & & & 9 & & $\%$ & $\%$ & $\%$ & $\%$ & $\%$ \\
\hline PAT 1 & no symptoms & 19 & 3.9 & 0 & 94.7 & 94.7 & 94.7 & 94.7 & 94.7 \\
\hline PAT 2 & complaints only & 66 & 13.6 & 0 & 96.8 & 93.4 & 91.5 & 89.5 & 87.3 \\
\hline PAT 3 & focal signs & 183 & 37.6 & 2 & 91.0 & 84.2 & 78.9 & 77.4 & 73.4 \\
\hline PAT 4 & increased ICP & 183 & 37.6 & 4 & 94.3 & 85.4 & 77.9 & 71.1 & 65.4 \\
\hline PAT 5 & $\begin{array}{l}\text { disturbance of } \\
\text { consciousness }\end{array}$ & 28 & 5.7 & 2 & 89.1 & 73.3 & 68.9 & 64.5 & 59.7 \\
\hline PAT 6 & coma & 7 & 1.4 & 3 & 85.7 & 85.7 & 57.1 & 42.9 & 28.6 \\
\hline PAT 7 & $\begin{array}{l}\text { respiratory } \\
\text { impairment }\end{array}$ & 1 & 0.2 & 2 & 100.0 & 100.0 & 100.0 & 100.0 & 100.0 \\
\hline & total & 487 & 100.0 & 13 & & & & & \\
\hline
\end{tabular}

$* 1$ Number of cases who lived more than 30 days.

$* 2$ Number of cases who died within 30 days. 
Mantel Chi-Square Test

\begin{tabular}{|c|c|c|c|c|c|c|c|}
\hline & PAT 1 & PAT 2 & PAT 3 & PAT 4 & PAT 5 & PAT 6 & PAT 7 \\
\hline PAT 1 & -1.00 & 0.39 & 2.56 & 3.49 & $\begin{array}{r}* \\
4.41\end{array}$ & $\begin{array}{r}* * \\
7.47\end{array}$ & 0.05 \\
\hline PAT 2 & 0.39 & -1.00 & $\begin{array}{r}* * \\
4.14\end{array}$ & $\begin{array}{r}* * \\
7.47 \\
\end{array}$ & $\begin{array}{r}* * * \\
7.30 \\
\end{array}$ & $\begin{array}{r}* * * \\
12.36\end{array}$ & 0.13 \\
\hline PAT 3 & 2.56 & $\begin{array}{r}* * \\
4.14\end{array}$ & -1.00 & 1.05 & 1.68 & $\begin{array}{r}* * \\
5.05\end{array}$ & 0.30 \\
\hline PAT 4 & 3.49 & $\begin{array}{r}* * \\
7.47 \\
\end{array}$ & 1.05 & -1.00 & 0.55 & 3.08 & 0.40 \\
\hline PAT 5 & $\begin{array}{r}* \\
4.41 \\
\end{array}$ & $\begin{array}{r}* * \\
7.30 \\
\end{array}$ & 1.68 & 0.55 & -1.00 & 1.08 & 0.46 \\
\hline PAT 6 & $\begin{array}{r}* * * \\
7.47 \\
\end{array}$ & $\begin{array}{r}* * * * \\
12.36 \\
\end{array}$ & $\begin{array}{r}* \\
5.05 \\
\end{array}$ & 3.08 & 1.08 & -1.00 & 0.86 \\
\hline PAT 7 & 0.05 & 0.13 & 0.30 & 0.40 & 0.46 & 0.86 & -1.00 \\
\hline
\end{tabular}

c) Oparation With or Without Radiation Therapy (1984-2000) : Cumulative Survival Rate

\begin{tabular}{|c|c|c|c|c|c|c|c|c|c|}
\hline & PATTERN & NO. OF C & $S \quad * 1$ & $* 2$ & 1 -YEAR & 2 -YEAR & 3 -YEAR & 4-YEAR & 5-YEAR \\
\hline & & & $\%$ & & $\%$ & $\%$ & $\%$ & $\%$ & $\%$ \\
\hline PAT 1 & $\begin{array}{l}\text { ope (biopsy) + } \\
\text { radiation }\end{array}$ & 28 & 6.0 & 0 & 76.5 & 66.6 & 66.6 & 61.1 & 55.5 \\
\hline PAT 2 & ope (biopsy) alone & 11 & 2.4 & 0 & 80.0 & 80.0 & 48.0 & 48.0 & 48.0 \\
\hline PAT 3 & $\begin{array}{l}\text { ope }(50-75 \% \text { resected }) \\
+ \text { radiation }\end{array}$ & 89 & 19.2 & 0 & 95.4 & 84.7 & 78.7 & 73.6 & 66.6 \\
\hline PAT 4 & $\begin{array}{l}\text { ope }(50-75 \% \text { resected }) \\
\text { alone }\end{array}$ & 24 & 5.2 & 6 & 61.7 & 43.4 & 38.0 & 38.0 & 29.5 \\
\hline PAT 5 & $\begin{array}{l}\text { ope }(\geqq 95 \% \\
\text { resected)+radiation }\end{array}$ & 168 & 36.2 & 0 & 98.8 & 92.3 & 86.1 & 80.7 & 74.0 \\
\hline PAT 6 & $\begin{array}{l}\text { ope }(\geqq 95 \% \\
\text { resected) alone }\end{array}$ & 144 & 31.0 & 3 & 95.0 & 91.8 & 89.1 & 87.1 & 87.1 \\
\hline PAT 7 & $\begin{array}{l}\text { ope (all cases) + } \\
\text { radiation }\end{array}$ & 290 & 61.2 & 0 & 95.7 & 87.4 & 81.4 & 76.2 & 69.6 \\
\hline PAT 8 & ope alone (all cases) & 184 & 38.8 & 10 & 89.9 & 84.2 & 79.9 & 78.4 & 77.5 \\
\hline
\end{tabular}

$* 1$ Number of cases who lived more than 30 days.

$* 2$ Number of cases who died within 30 days. 
Mantel Chi-Square Test

\begin{tabular}{|c|c|c|c|c|c|c|c|c|}
\hline & PAT 1 & PAT 2 & PAT 3 & PAT 4 & PAT 5 & PAT 6 & PAT 7 & PAT 8 \\
\hline PAT 1 & -1.00 & 0.02 & 1.85 & 1.81 & $\begin{array}{r}* \\
6.46\end{array}$ & $\begin{array}{r}* * * \\
12.22\end{array}$ & 3.80 & $\begin{array}{r}* \\
4.41\end{array}$ \\
\hline PAT 2 & 0.02 & -1.00 & 1.78 & 0.78 & $\begin{array}{r}* \\
5.04 \\
\end{array}$ & $\begin{array}{r}* * \\
6.94 \\
\end{array}$ & 2.85 & 2.31 \\
\hline PAT 3 & 1.85 & 1.78 & -1.00 & $\begin{array}{r}* * * * \\
12.48 \\
\end{array}$ & 1.66 & $\begin{array}{r}* * \\
8.93 \\
\end{array}$ & 0.24 & 1.54 \\
\hline PAT 4 & 1.81 & 0.78 & $\begin{array}{r}* * * \\
12.48 \\
\end{array}$ & -1.00 & $\begin{array}{r}* * * * \\
29.08 \\
\end{array}$ & $\begin{array}{r}* * * * \\
32.56 \\
\end{array}$ & $\begin{array}{r}* * * * \\
22.89 \\
\end{array}$ & $\begin{array}{r}* * * \\
17.88 \\
\end{array}$ \\
\hline PAT 5 & $\begin{array}{r}* \\
6.46 \\
\end{array}$ & $\begin{array}{r}* \\
5.04 \\
\end{array}$ & 1.66 & $\begin{array}{r}* * * \\
29.08 \\
\end{array}$ & -1.00 & 3.79 & 1.16 & 0.02 \\
\hline PAT 6 & $\begin{array}{r}* * * \\
12.22 \\
\end{array}$ & $\begin{array}{r}* * \\
6.94 \\
\end{array}$ & $\begin{array}{r}* * * \\
8.93 \\
\end{array}$ & $\begin{array}{r}* * * \\
32.56 \\
\end{array}$ & 3.79 & -1.00 & $\begin{array}{r}* * \\
8.84 \\
\end{array}$ & $\begin{array}{r}* \\
4.02 \\
\end{array}$ \\
\hline PAT 7 & 3.80 & 2.85 & 0.24 & $\begin{array}{r}* * * * \\
22.89 \\
\end{array}$ & 1.16 & $\begin{array}{r}* * \\
8.84 \\
\end{array}$ & -1.00 & 0.77 \\
\hline PAT 8 & $\begin{array}{r}* * \\
4.41 \\
\end{array}$ & 2.31 & 1.54 & $\begin{array}{r}* * * \\
17.88 \\
\end{array}$ & 0.02 & $\begin{array}{r}* \\
4.02 \\
\end{array}$ & 0.77 & -1.00 \\
\hline
\end{tabular}

\section{6) Anaplastic Ependymoma}

\section{a) Operation (1984-2000): Cumulative Survival Rate}

\begin{tabular}{|c|c|c|c|c|c|c|c|c|c|}
\hline \multicolumn{2}{|r|}{ PATTERN } & \multicolumn{2}{|c|}{ NO. OF CASES $* 1$} & \multirow[t]{2}{*}{$* 2$} & \multirow{2}{*}{$\frac{1-Y E A R}{\%}$} & \multirow{2}{*}{$\frac{2-\text { YEAR }}{\%}$} & \multirow{2}{*}{$\frac{3 \text {-YEAR }}{\%}$} & \multirow{2}{*}{$\begin{array}{r}4-\mathrm{YEAR} \\
\%\end{array}$} & \multirow{2}{*}{$\begin{array}{r}5 \text {-YEAR } \\
\%\end{array}$} \\
\hline & & & $\%$ & & & & & & \\
\hline PAT 0 & none & 6 & 4.1 & 1 & 81.8 & 81.8 & 81.8 & 81.8 & 81.8 \\
\hline PAT 1 & decompression & 6 & 4.1 & 3 & 66.7 & 66.7 & 50.0 & 33.3 & 33.3 \\
\hline PAT 2 & biopsy or partial & 19 & 13.1 & 2 & 51.4 & 21.1 & 14.1 & 14.1 & 7.0 \\
\hline PAT 3 & $50 \%$ resected & 12 & 8.3 & 1 & 75.0 & 33.3 & 14.3 & 14.3 & 14.3 \\
\hline PAT 4 & $75 \%$ resected & 35 & 24.1 & 1 & 72.7 & 48.5 & 33.0 & 33.0 & 28.3 \\
\hline PAT 5 & $95 \%$ resected & 43 & 29.7 & 0 & 90.2 & 61.0 & 52.7 & 49.9 & 38.2 \\
\hline PAT 6 & total removal & 24 & 16.6 & 0 & 87.0 & 82.3 & 72.6 & 67.4 & 55.1 \\
\hline & total & 145 & 100.0 & 8 & & & & & \\
\hline
\end{tabular}

*1 Number of cases who lived more than 30 days.

*2 Number of cases who died within 30 days.

\section{Mantel Chi-Square Test}

\begin{tabular}{|c|c|c|c|c|c|c|c|}
\hline & PAT 0 & PAT 1 & PAT 2 & PAT 3 & PAT 4 & PAT 5 & PAT 6 \\
\hline PAT 0 & -1.00 & 1.47 & $\begin{array}{r}* \\
4.07 \\
\end{array}$ & 2.58 & 2.25 & 1.29 & 0.56 \\
\hline PAT 1 & 1.47 & -1.00 & 1.11 & 0.50 & 0.04 & 0.30 & 0.94 \\
\hline PAT 2 & $\begin{array}{r}* \\
4.07 \\
\end{array}$ & 1.11 & -1.00 & 0.33 & 1.94 & $\begin{array}{r}* * \\
6.66 \\
\end{array}$ & $\begin{array}{r}* * \\
8.50 \\
\end{array}$ \\
\hline PAT 3 & 2.58 & 0.50 & 0.33 & -1.00 & 0.35 & 2.85 & $\begin{array}{r}* \\
5.71 \\
\end{array}$ \\
\hline PAT 4 & 2.25 & 0.04 & 1.94 & 0.35 & -1.00 & 1.34 & 3.45 \\
\hline PAT 5 & 1.29 & 0.30 & $\begin{array}{r}* * \\
6.66 \\
\end{array}$ & 2.85 & 1.34 & -1.00 & 0.98 \\
\hline PAT 6 & 0.56 & 0.94 & $\begin{array}{r}* * \\
8.50 \\
\end{array}$ & $\begin{array}{r}* \\
5.71 \\
\end{array}$ & 3.45 & 0.98 & -1.00 \\
\hline
\end{tabular}


b) Clinical Grading (1984-2000) : Cumulative Survival Rate

\begin{tabular}{|c|c|c|c|c|c|c|c|c|c|}
\hline & PATTERN & NO. OF C & $\mathrm{ES} \quad * 1$ & $* 2$ & 1-YEAR & 2-YEAR & 3-YEAR & 4-YEAR & 5-YEAR \\
\hline & & & $\%$ & & $\%$ & $\%$ & $\%$ & $\%$ & $\%$ \\
\hline PAT 1 & no symptoms & 3 & 2.1 & 0 & 66.7 & 66.7 & 66.7 & 66.7 & 66.7 \\
\hline PAT 2 & complaints only & 5 & 3.5 & 0 & 100.0 & 100.0 & 100.0 & 100.0 & 42.9 \\
\hline PAT 3 & focal signs & 58 & 40.3 & 3 & 83.9 & 54.7 & 37.5 & 30.7 & 30.7 \\
\hline PAT 4 & increased ICP & 58 & 40.3 & 3 & 80.2 & 58.7 & 46.3 & 46.3 & 37.3 \\
\hline PAT 5 & $\begin{array}{l}\text { disturbance of } \\
\text { consciousness }\end{array}$ & 16 & 11.1 & 1 & 41.9 & 28.0 & 28.0 & 28.0 & 9.3 \\
\hline PAT 6 & coma & 4 & 2.8 & 1 & 71.4 & 35.7 & 35.7 & 35.7 & 35.7 \\
\hline PAT 7 & $\begin{array}{l}\text { respiratory } \\
\text { impairment }\end{array}$ & 0 & 0.0 & 0 & - & - & - & - & - \\
\hline & total & 144 & 100.0 & 8 & & & & & \\
\hline
\end{tabular}

*1 Number of cases who lived more than 30 days.

$* 2$ Number of cases who died within 30 days.

Mantel Chi-Square Test : No significancy Detected between Each Two of PATs

c) Oparation With or Without Radiation Therapy (1984-2000): Cumulative Survival Rate

\begin{tabular}{|c|c|c|c|c|c|c|c|c|c|}
\hline & PATTERN & NO. OF C & $* 1$ & $* 2$ & 1-YEAR & 2-YEAR & 3-YEAR & 4-YEAR & 5-YEAR \\
\hline & & & $\%$ & & $\%$ & $\%$ & $\%$ & $\%$ & $\%$ \\
\hline PAT 1 & $\begin{array}{l}\text { ope (biopsy) + } \\
\text { radiation }\end{array}$ & 17 & 12.9 & 0 & 51.5 & 24.0 & 16.0 & 16.0 & 8.0 \\
\hline PAT 2 & ope (biopsy) alone & 2 & 1.5 & 2 & 50.0 & 0.0 & - & - & - \\
\hline PAT 3 & $\begin{array}{l}\text { ope }(50-75 \% \text { resected }) \\
+ \text { radiation }\end{array}$ & 37 & 28.0 & 0 & 80.3 & 50.0 & 33.3 & 33.3 & 28.9 \\
\hline PAT 4 & $\begin{array}{l}\text { ope }(50-75 \% \text { resected }) \\
\text { alone }\end{array}$ & 10 & 7.6 & 2 & 47.4 & 20.3 & 0.0 & - & - \\
\hline PAT 5 & $\begin{array}{l}\text { ope }(\geqq 95 \% \text { resected })+ \\
\text { radiation }\end{array}$ & 53 & 40.2 & 0 & 92.2 & 68.9 & 60.0 & 55.4 & 43.1 \\
\hline PAT 6 & $\begin{array}{l}\text { ope }(\geqq 95 \% \\
\text { resected) alone }\end{array}$ & 13 & 9.8 & 0 & 75.0 & 65.6 & 56.3 & 56.3 & 56.3 \\
\hline PAT 7 & $\begin{array}{l}\text { ope (all cases) + } \\
\text { radiation }\end{array}$ & 112 & 81.2 & 0 & 80.6 & 55.5 & 43.8 & 40.4 & 31.8 \\
\hline PAT 8 & ope alone (all cases) & 26 & 18.8 & 7 & 63.3 & 44.5 & 34.6 & 34.6 & 34.6 \\
\hline
\end{tabular}

*1 Number of cases who lived more than 30 days.

$* 2$ Number of cases who died within 30 days. 
Mantel Chi-Square Test

\begin{tabular}{|l|r|r|r|r|r|r|r|r|}
\hline & PAT 1 & \multicolumn{1}{|c|}{ PAT 2 } & \multicolumn{1}{c|}{ PAT 3 } & PAT 4 & PAT 5 & PAT 6 & \multicolumn{1}{c|}{ PAT 7 } & PAT 8 \\
\hline PAT 1 & -1.00 & 0.07 & 2.37 & 0.09 & 8.56 & 3.09 & 4.18 & 1.21 \\
\hline PAT 2 & 0.07 & -1.00 & 1.00 & 0.03 & 3.11 & 1.30 & 1.34 & 0.48 \\
\hline PAT 3 & 2.37 & 1.00 & -1.00 & 2.50 & 2.29 & 0.81 & 0.13 & 0.04 \\
\hline PAT 4 & 0.09 & 0.03 & 2.50 & -1.00 & 9.04 & 2.67 & 4.18 & 1.06 \\
\hline PAT 5 & $\begin{array}{r}* * \\
* 6\end{array}$ & 3.11 & 2.29 & 9.04 & -1.00 & 0.01 & 2.08 & 2.14 \\
\hline PAT 6 & 3.09 & 1.30 & 0.81 & 2.67 & 0.01 & -1.00 & 0.73 & 0.78 \\
\hline PAT 7 & 4.18 & 1.34 & 0.13 & 4.18 & 2.08 & 0.73 & -1.00 & 0.25 \\
\hline PAT 8 & 1.21 & 0.48 & 0.04 & 1.06 & 2.14 & 0.78 & 0.25 & -1.00 \\
\hline
\end{tabular}

\section{7) Oligodendroglioma}

\section{a) Operation (1984-2000): Cumulative Survival Rate}

\begin{tabular}{|c|c|c|c|c|c|c|c|c|c|}
\hline & PATTERN & NO. OF C & $* 1$ & $* 2$ & 1-YEAR & 2-YEAR & 3-YEAR & 4-YEAR & 5-YEAR \\
\hline & & & $\%$ & & $\%$ & $\%$ & $\%$ & $\%$ & $\%$ \\
\hline PAT 0 & none & 26 & 4.6 & 0 & 96.1 & 87.7 & 78.7 & 74.0 & 68.7 \\
\hline PAT 1 & decompression & 6 & 1.1 & 0 & 83.3 & 66.7 & 66.7 & 66.7 & 66.7 \\
\hline PAT 2 & biopsy or partial & 89 & 15.7 & 2 & 91.8 & 90.5 & 84.7 & 83.2 & 76.4 \\
\hline PAT 3 & $50 \%$ resected & 63 & 11.1 & 0 & 93.5 & 88.3 & 79.1 & 75.1 & 70.6 \\
\hline PAT 4 & $75 \%$ resected & 165 & 29.2 & 1 & 96.3 & 92.2 & 85.5 & 82.1 & 78.3 \\
\hline PAT 5 & $95 \%$ resected & 142 & 25.1 & 0 & 98.5 & 93.8 & 90.3 & 89.4 & 87.3 \\
\hline PAT 6 & total removal & 75 & 13.3 & 0 & 94.4 & 89.8 & 89.8 & 87.9 & 87.9 \\
\hline & total & 566 & 100.0 & 3 & & & & & \\
\hline
\end{tabular}

$* 1$ Number of cases who lived more than 30 days.

$* 2$ Number of cases who died within 30 days.

\section{Mantel Chi-Square Test}

\begin{tabular}{|l|r|r|r|r|r|r|r|}
\hline & \multicolumn{1}{|c|}{ PAT 0 } & \multicolumn{1}{c|}{ PAT 1 } & \multicolumn{1}{c|}{ PAT 2 } & \multicolumn{1}{c|}{ PAT 3 } & \multicolumn{1}{c|}{ PAT 4 } & \multicolumn{1}{c|}{ PAT 5 } & \multicolumn{1}{c|}{ PAT 6 } \\
\hline PAT 0 & -1.00 & 0.13 & 0.47 & 0.01 & 0.90 & 4.40 & 2.96 \\
\hline PAT 1 & 0.13 & -1.00 & 0.63 & 0.23 & 1.00 & 2.85 & 1.91 \\
\hline PAT 2 & 0.47 & 0.63 & -1.00 & 0.52 & 0.12 & 3.43 & 1.86 \\
\hline PAT 3 & 0.01 & 0.23 & 0.52 & -1.00 & 1.26 & 6.43 & 3.72 \\
\hline PAT 4 & 0.90 & 1.00 & 0.12 & 1.26 & -1.00 & 2.93 & 1.36 \\
\hline PAT 5 & 4.40 & 2.85 & 3.43 & 6.43 & 2.93 & -1.00 & 0.03 \\
\hline PAT 6 & 2.96 & 1.91 & 1.86 & 3.72 & 1.36 & 0.03 & -1.00 \\
\hline
\end{tabular}


b) Clinical Grading (1984-2000) : Cumulative Survival Rate

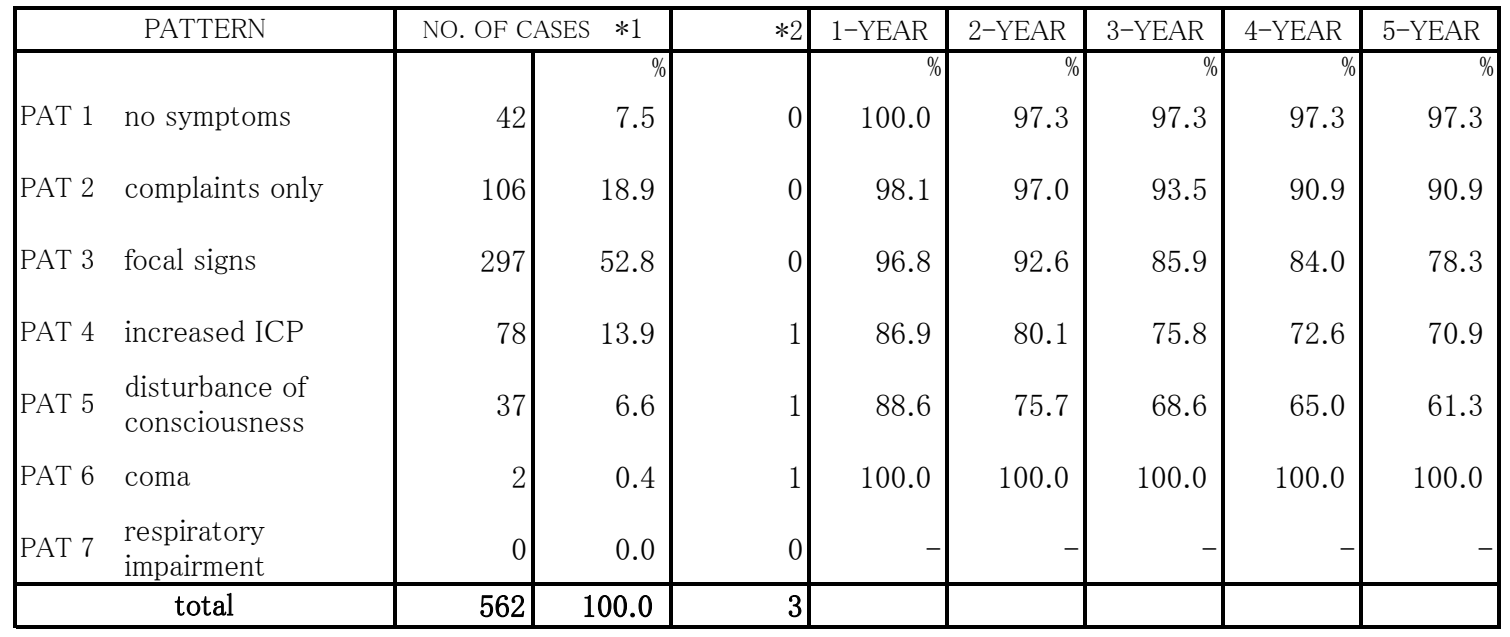

*1 Number of cases who lived more than 30 days.

$* 2$ Number of cases who died within 30 days.

\section{Mantel Chi-Square Test}

\begin{tabular}{|c|c|c|c|c|c|c|c|}
\hline & PAT 1 & PAT 2 & PAT 3 & PAT 4 & PAT 5 & PAT 6 & PAT 7 \\
\hline PAT 1 & -1.00 & 1.27 & $\begin{array}{r}* \\
5.53\end{array}$ & $\begin{array}{r}* * \\
9.01\end{array}$ & $\begin{array}{r}\text { *** } \\
12.05\end{array}$ & 0.05 & -1.00 \\
\hline PAT 2 & 1.27 & -1.00 & $\begin{array}{r}* \\
5.55\end{array}$ & $\begin{array}{r}\text { *** } \\
10.99\end{array}$ & $\begin{array}{r}\text { *** } \\
14.75\end{array}$ & 0.19 & -1.00 \\
\hline PAT 3 & $\begin{array}{r}* * \\
5.53\end{array}$ & $\begin{array}{r}* * \\
5.55\end{array}$ & -1.00 & 3.13 & $\begin{array}{r}* * \\
5.77\end{array}$ & 0.47 & -1.00 \\
\hline PAT 4 & $\begin{array}{r}* * * \\
9.01 \\
\end{array}$ & $\begin{array}{r}* * * * \\
10.99 \\
\end{array}$ & 3.13 & -1.00 & 0.58 & 0.65 & -1.00 \\
\hline PAT 5 & $\begin{array}{r}* * * * \\
12.05 \\
\end{array}$ & $\begin{array}{r}* * * * \\
14.75 \\
\end{array}$ & $\begin{array}{r}* \\
5.77 \\
\end{array}$ & 0.58 & -1.00 & 0.89 & -1.00 \\
\hline PAT 6 & 0.05 & 0.19 & 0.47 & 0.65 & 0.89 & -1.00 & -1.00 \\
\hline PAT 7 & -1.00 & -1.00 & -1.00 & -1.00 & -1.00 & -1.00 & -1.00 \\
\hline
\end{tabular}

\section{c) Extension (1984-2000): Cumulative Survival Rate}

\begin{tabular}{|c|c|c|c|c|c|c|c|c|c|}
\hline & PATTERN & NO. OF C & $\mathrm{ES} * 1$ & $* 2$ & 1-YEAR & 2-YEAR & 3-YEAR & 4-YEAR & 5-YEAR \\
\hline & & & $\%$ & & $\%$ & $\%$ & $\%$ & $\%$ & $\%$ \\
\hline PAT 1 & one side & 344 & 61.6 & 1 & 96.7 & 93.4 & 89.4 & 87.9 & 85.4 \\
\hline PAT 2 & ventricular system & 83 & 14.9 & 1 & 95.0 & 86.7 & 80.2 & 72.6 & 68.3 \\
\hline PAT 3 & central & 61 & 10.9 & 0 & 95.0 & 87.8 & 81.9 & 79.8 & 75.1 \\
\hline PAT 4 & opposite side & 50 & 9.0 & 1 & 93.9 & 91.7 & 77.8 & 75.2 & 66.4 \\
\hline PAT 5 & $\begin{array}{l}\text { supra }+ \\
\text { infratentorial }\end{array}$ & 1 & 0.2 & 0 & 100.0 & 100.0 & 100.0 & 100.0 & 100.0 \\
\hline PAT 6 & other & 19 & 3.4 & 0 & 83.8 & 83.8 & 83.8 & 83.8 & 83.8 \\
\hline & total & 558 & 100.0 & 3 & & & & & \\
\hline
\end{tabular}

*1 Number of cases who lived more than 30 days.

$* 2$ Number of cases who died within 30 days. 
Mantel Chi-Square Test

\begin{tabular}{|l|r|r|r|r|r|r|}
\hline & PAT 1 & PAT 2 & PAT 3 & PAT 4 & PAT 5 & PAT 6 \\
\hline PAT 1 & -1.00 & 8.82 & 3.16 & 7.26 & 0.15 & 0.36 \\
\hline PAT 2 & $\begin{array}{r}* * \\
8.82\end{array}$ & -1.00 & 0.43 & 0.00 & 0.36 & 0.47 \\
\hline PAT 3 & 3.16 & 0.43 & -1.00 & 0.42 & 0.27 & 0.12 \\
\hline PAT 4 & $\begin{array}{r}* * \\
\text { Pै }\end{array}$ & 0.00 & 0.42 & -1.00 & 0.38 & 0.53 \\
\hline PAT 5 & 0.15 & 0.36 & 0.27 & 0.38 & -1.00 & 0.15 \\
\hline PAT 6 & 0.36 & 0.47 & 0.12 & 0.53 & 0.15 & -1.00 \\
\hline
\end{tabular}

d) Oparation With or Without Radiation Therapy (1984-2000) : Cumulative Survival Rate

\begin{tabular}{|c|c|c|c|c|c|c|c|c|c|}
\hline & PATTERN & NO. OF & ES *1 & $* 2$ & 1-YEAR & 2-YEAR & 3-YEAR & 4-YEAR & 5-YEAR \\
\hline & & & 9 & & $\%$ & $\%$ & $\%$ & $\%$ & $\%$ \\
\hline PAT 1 & $\begin{array}{l}\text { ope (biopsy) + } \\
\text { radiation }\end{array}$ & 70 & 13.2 & 0 & 91.0 & 89.4 & 84.0 & 82.0 & 73.4 \\
\hline PAT 2 & ope (biopsy) alone & 19 & 3.6 & 2 & 94.4 & 94.4 & 87.4 & 87.4 & 87.4 \\
\hline PAT 3 & $\begin{array}{l}\text { ope }(50-75 \% \text { resected }) \\
+ \text { radiation }\end{array}$ & 170 & 32.0 & 1 & 94.5 & 89.3 & 80.6 & 78.1 & 74.4 \\
\hline PAT 4 & $\begin{array}{l}\text { ope }(50-75 \% \text { resected }) \\
\text { alone }\end{array}$ & 57 & 10.7 & 0 & 98.2 & 96.3 & 92.2 & 87.9 & 83.1 \\
\hline PAT 5 & $\begin{array}{l}\text { ope }(\geqq 95 \% \text { resected }) \\
+ \text { radiation }\end{array}$ & 139 & 26.1 & 0 & 98.5 & 93.5 & 90.8 & 89.8 & 88.8 \\
\hline PAT 6 & $\begin{array}{l}\text { ope }(\geqq 95 \% \\
\text { resected) alone }\end{array}$ & 77 & 14.5 & 0 & 96.0 & 91.6 & 90.0 & 88.1 & 86.0 \\
\hline PAT 7 & $\begin{array}{l}\text { ope (all cases) + } \\
\text { radiation }\end{array}$ & 384 & 71.4 & 1 & 95.1 & 90.4 & 84.6 & 82.8 & 79.3 \\
\hline PAT 8 & ope alone (all cases) & 154 & 28.6 & 2 & 96.6 & 93.8 & 90.6 & 88.0 & 85.0 \\
\hline
\end{tabular}

*1 Number of cases who lived more than 30 days.

$* 2$ Number of cases who died within 30 days.

\section{Mantel Chi-Square Test}

\begin{tabular}{|l|r|r|r|r|r|r|r|r|}
\hline & PAT 1 & PAT 2 & \multicolumn{1}{c|}{ PAT 3 } & PAT 4 & PAT 5 & PAT 6 & PAT 7 & PAT 8 \\
\hline PAT 1 & -1.00 & 0.91 & 0.00 & 1.48 & 5.66 & 2.14 & 0.69 & 3.01 \\
\hline PAT 2 & 0.91 & -1.00 & 0.93 & 0.04 & 0.06 & 0.01 & 0.43 & 0.02 \\
\hline PAT 3 & 0.00 & 0.93 & -1.00 & 1.84 & 7.26 & 2.69 & 1.10 & $\begin{array}{r}* \\
\end{array}$ \\
\hline PAT 4 & 1.48 & 0.04 & 1.84 & -1.00 & 0.58 & 0.03 & 0.62 & 0.02 \\
\hline PAT 5 & 5.66 & 0.06 & 7.26 & 0.58 & -1.00 & 0.31 & 4.52 & 0.58 \\
\hline PAT 6 & 2.14 & 0.01 & 2.69 & 0.03 & 0.31 & -1.00 & 1.19 & 0.00 \\
\hline PAT 7 & 0.69 & 0.43 & 1.10 & 0.62 & 4.52 & 1.19 & -1.00 & 1.97 \\
\hline PAT 8 & 3.01 & 0.02 & 4.38 & 0.02 & 0.58 & 0.00 & 1.97 & -1.00 \\
\hline
\end{tabular}


18) Anaplastic Oligodendroglioma

a) Operation (1984-2000): Cumulative Survival Rate

\begin{tabular}{|ll|r|r|r|r|r|r|r|r|}
\hline & PATTERN & \multicolumn{2}{|c|}{ NO. OF CASES $* 1$} & $* 2$ & 1-YEAR & 2-YEAR & 3-YEAR & 4-YEAR & 5-YEAR \\
\hline PAT 0 none & 5 & 3.4 & 0 & 80.0 & 57.1 & 57.1 & 57.1 & 28.6 \\
PAT 1 decompression & 0 & 0.0 & 0 & - & - & - & - & \\
PAT 2 biopsy or partial & 14 & 9.5 & 0 & 64.3 & 50.0 & 42.3 & 25.4 & 15.2 \\
PAT 3 50\% resected & 19 & 12.9 & 0 & 55.6 & 39.7 & 26.5 & 26.5 & 26.5 \\
PAT 4 75\% resected & 38 & 25.9 & 0 & 88.7 & 88.7 & 85.1 & 81.1 & 67.5 \\
PAT 5 95\% resected & 45 & 30.6 & 1 & 90.7 & 88.0 & 78.6 & 64.6 & 64.6 \\
PAT 6 total removal & 26 & 17.7 & 0 & 96.0 & 96.0 & 90.7 & 90.7 & 90.7 \\
\hline \multicolumn{2}{r|}{ total } & 147 & 100.0 & 1 & & & & & \\
\hline
\end{tabular}

*1 Number of cases who lived more than 30 days.

$* 2$ Number of cases who died within 30 days.

Manatel Chi-Square Test

\begin{tabular}{|c|c|c|c|c|c|c|c|}
\hline & PAT 0 & PAT 1 & PAT 2 & PAT 3 & PAT 4 & PAT 5 & PAT 6 \\
\hline PAT 0 & -1.00 & -1.00 & 0.35 & 0.19 & 2.04 & 2.15 & $\begin{array}{r}* * \\
7.05 \\
\end{array}$ \\
\hline PAT 1 & -1.00 & -1.00 & -1.00 & -1.00 & -1.00 & -1.00 & -1.00 \\
\hline PAT 2 & 0.35 & -1.00 & -1.00 & 0.01 & $\begin{array}{r}* * \\
9.78 \\
\end{array}$ & $\begin{array}{r}* * \\
8.15 \\
\end{array}$ & $\begin{array}{r}\text { **** } \\
15.26 \\
\end{array}$ \\
\hline PAT 3 & 0.19 & -1.00 & 0.01 & -1.00 & $\begin{array}{r}* * \\
8.03 \\
\end{array}$ & $\begin{array}{r}* * \\
8.50 \\
\end{array}$ & $\begin{array}{r}* * * \\
11.96 \\
\end{array}$ \\
\hline PAT 4 & 2.04 & -1.00 & $\begin{array}{r}* * \\
9.78 \\
\end{array}$ & $\begin{array}{r}* * \\
8.03 \\
\end{array}$ & -1.00 & 0.14 & 2.40 \\
\hline PAT 5 & 2.15 & -1.00 & $\begin{array}{r}* * * \\
8.15 \\
\end{array}$ & $\begin{array}{r}* * * \\
8.50 \\
\end{array}$ & 0.14 & -1.00 & 3.29 \\
\hline PAT 6 & $\begin{array}{r}* * \\
7.05 \\
\end{array}$ & -1.00 & $\begin{array}{r}* * * \\
15.26 \\
\end{array}$ & $\begin{array}{r}* * * \\
11.96 \\
\end{array}$ & 2.40 & 3.29 & -1.00 \\
\hline
\end{tabular}

b) Clinical Grading (1984-2000) : Cumulative Survival Rate

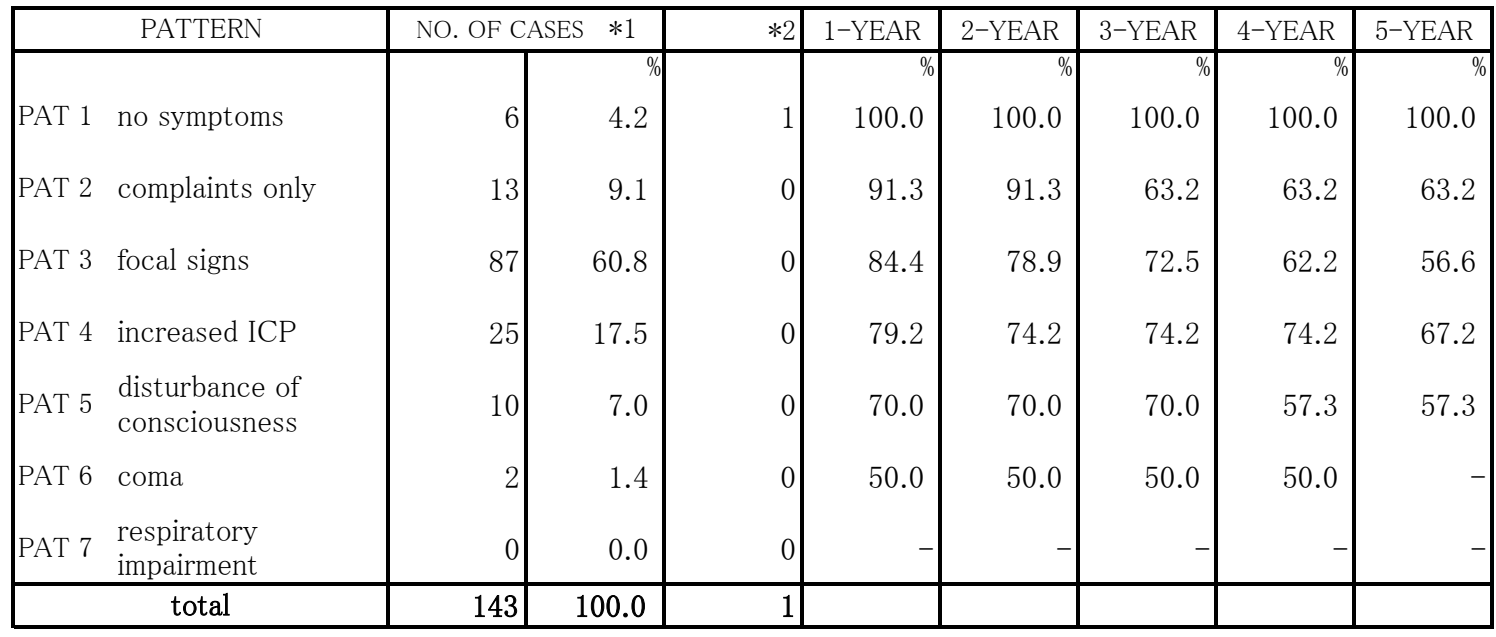

*1 Number of cases who lived more than 30 days.

$* 2$ Number of cases who died within 30 days.

Mantel Chi-Square Test : No Significancy Detected between Each Two of PATs 
c) Extension (1984-2000): Cumulative Survival Rate

\begin{tabular}{|c|c|c|c|c|c|c|c|c|c|}
\hline & PATTERN & NO. OF C & ES $* 1$ & $* 2$ & 1-YEAR & 2-YEAR & 3-YEAR & 4-YEAR & 5-YEAR \\
\hline & & & $\%$ & & $\%$ & $\%$ & $\%$ & $\%$ & $\%$ \\
\hline PAT 1 & one side & 85 & 57.8 & 0 & 85.1 & 82.2 & 74.0 & 65.4 & 59.7 \\
\hline PAT 2 & ventricular system & 17 & 11.6 & 0 & 87.5 & 87.5 & 87.5 & 87.5 & 74.0 \\
\hline PAT 3 & central & 22 & 15.0 & 0 & 81.4 & 76.1 & 76.1 & 62.3 & 55.4 \\
\hline PAT 4 & opposite side & 16 & 10.9 & 0 & 74.2 & 67.1 & 58.7 & 58.7 & 58.7 \\
\hline PAT 5 & $\begin{array}{l}\text { supra }+ \\
\text { infratentorial }\end{array}$ & 1 & 0.7 & 0 & 0.0 & - & - & - & - \\
\hline PAT 6 & other & 6 & 4.1 & 0 & 100.0 & 66.7 & 50.0 & 50.0 & 50.0 \\
\hline & total & 147 & 100.0 & 0 & & & & & \\
\hline
\end{tabular}

*1 Number of cases who lived more than 30 days.

$* 2$ Number of cases who died within 30 days.

Mantel Chi-Square Test : No Significancy Detected between Each Two of PATs

d) Oparation With or Without Radiation Therapy (1984-2000): Cumulative Survival Rate

\begin{tabular}{|c|c|c|c|c|c|c|c|c|c|}
\hline & PATTERN & NO. OF C & $S \quad * 1$ & $* 2$ & 1-YEAR & 2-YEAR & 3-YEAR & 4-YEAR & 5-YEAR \\
\hline & & & $\%$ & & $\%$ & $\%$ & $\%$ & $\%$ & $\%$ \\
\hline PAT 1 & $\begin{array}{l}\text { ope (biopsy) + } \\
\text { radiation }\end{array}$ & 14 & 9.9 & 0 & 64.3 & 50.0 & 42.3 & 25.4 & 15.2 \\
\hline PAT 2 & ope (biopsy) alone & 0 & 0.0 & 0 & - & - & - & - & - \\
\hline PAT 3 & $\begin{array}{l}\text { ope }(50-75 \% \text { resected }) \\
+ \text { radiation }\end{array}$ & 46 & 32.4 & 0 & 83.5 & 80.5 & 73.5 & 73.5 & 61.3 \\
\hline PAT 4 & $\begin{array}{l}\text { ope }(50-75 \% \text { resected }) \\
\text { alone }\end{array}$ & 11 & 7.7 & 0 & 54.5 & 45.5 & 45.5 & 32.5 & 32.5 \\
\hline PAT 5 & $\begin{array}{l}\text { ope }(\geqq 95 \% \text { resected }) \\
+ \text { radiation }\end{array}$ & 59 & 41.5 & 0 & 94.6 & 92.6 & 82.8 & 72.3 & 72.3 \\
\hline PAT 6 & $\begin{array}{l}\text { ope }(\geqq 95 \% \\
\text { resected) alone }\end{array}$ & 12 & 8.5 & 1 & 83.3 & 83.3 & 83.3 & 83.3 & 83.3 \\
\hline PAT 7 & $\begin{array}{l}\text { ope }(\text { all cases })+ \\
\text { radiation }\end{array}$ & 119 & 83.8 & 0 & 86.7 & 82.5 & 73.9 & 66.0 & 60.2 \\
\hline PAT 8 & ope alone (all cases) & 23 & 16.2 & 1 & 69.6 & 65.1 & 65.1 & 58.9 & 58.9 \\
\hline
\end{tabular}

*1 Number of cases who lived more than 30 days.

*2 Number of cases who died within 30 days. 
Mantel Chi-Square Test

\begin{tabular}{|c|c|c|c|c|c|c|c|c|}
\hline & PAT 1 & PAT 2 & PAT 3 & PAT 4 & PAT 5 & PAT 6 & PAT 7 & PAT 8 \\
\hline PAT 1 & -1.00 & -1.00 & $\begin{array}{r}* \\
6.58 \\
\end{array}$ & 0.12 & $\begin{array}{r}* * * \\
13.60 \\
\end{array}$ & $\begin{array}{r}* \\
5.83 \\
\end{array}$ & $\begin{array}{r}* * \\
8.41 \\
\end{array}$ & 2.82 \\
\hline PAT 2 & -1.00 & -1.00 & -1.00 & -1.00 & -1.00 & -1.00 & -1.00 & -1.00 \\
\hline PAT 3 & $\begin{array}{r}* \\
6.58 \\
\end{array}$ & -1.00 & -1.00 & 3.10 & 1.24 & 0.81 & 0.00 & 0.29 \\
\hline PAT 4 & 0.12 & -1.00 & 3.10 & -1.00 & $\begin{array}{r}* * \\
7.53 \\
\end{array}$ & 3.22 & $\begin{array}{r}* \\
3.86 \\
\end{array}$ & 0.98 \\
\hline PAT 5 & $\begin{array}{r}* * * \\
13.60 \\
\end{array}$ & -1.00 & 1.24 & $\begin{array}{r}* * \\
7.53 \\
\end{array}$ & -1.00 & 0.10 & 1.95 & 2.25 \\
\hline PAT 6 & $\begin{array}{r}* \\
5.83\end{array}$ & -1.00 & 0.81 & 3.22 & 0.10 & -1.00 & 1.03 & 1.26 \\
\hline PAT 7 & $\begin{array}{r}* * \\
8.41 \\
\end{array}$ & -1.00 & 0.00 & $\begin{array}{r}* * \\
3.86 \\
\end{array}$ & 1.95 & 1.03 & -1.00 & 0.32 \\
\hline PAT 8 & 2.82 & -1.00 & 0.29 & 0.98 & 2.25 & 1.26 & 0.32 & -1.00 \\
\hline
\end{tabular}




\section{Survival Rate by Age}

\section{1) All Glioma (1984-2000): Cumulative Survival Rate}

\begin{tabular}{|c|c|c|c|c|c|c|c|c|c|}
\hline \multicolumn{2}{|r|}{ PATTERN } & \multicolumn{2}{|c|}{ NO. OF CASES $* 1$} & \multirow[t]{2}{*}{$* 2$} & \multirow{2}{*}{$\frac{1 \text {-YEAR }}{\%}$} & \multirow{2}{*}{$\begin{array}{r}2 \text {-YEAR } \\
\%\end{array}$} & \multirow{2}{*}{$\frac{3 \text {-YEAR }}{\%}$} & \multirow{2}{*}{$\begin{array}{r}4 \text {-YEAR } \\
\%\end{array}$} & \multirow{2}{*}{$\frac{5 \text {-YEAR }}{\%}$} \\
\hline & & & $\%$ & & & & & & \\
\hline PAT & $0-4$ & 833 & 5.4 & 40 & 82.7 & 73.4 & 67.9 & 65.2 & 62.2 \\
\hline PAT & $5-9$ & 818 & 5.3 & 14 & 79.1 & 66.2 & 61.6 & 57.6 & 55.3 \\
\hline PAT & $10-14$ & 713 & 4.6 & 16 & 86.3 & 75.2 & 70.4 & 68.1 & 64.6 \\
\hline PAT & $15-24$ & 1,159 & 7.5 & 14 & 88.5 & 76.6 & 70.6 & 66.4 & 64.2 \\
\hline PAT & $25-34$ & 1,475 & 9.6 & 14 & 89.0 & 74.8 & 66.7 & 61.4 & 56.7 \\
\hline PAT & $35-44$ & 2,056 & 13.3 & 29 & 87.0 & 71.8 & 62.8 & 57.9 & 53.0 \\
\hline PAT & $45-54$ & 2,397 & 15.5 & 37 & 77.4 & 52.7 & 44.2 & 39.8 & 36.8 \\
\hline PAT & $55-64$ & 2,826 & 18.3 & 63 & 63.2 & 35.0 & 26.2 & 22.1 & 18.8 \\
\hline PAT & $65-74$ & 2,357 & 15.3 & 84 & 48.3 & 20.8 & 13.9 & 11.5 & 9.9 \\
\hline PAT 1 & $75-84$ & 754 & 4.9 & 38 & 32.2 & 13.1 & 7.6 & 5.7 & 5.7 \\
\hline PAT 1 & $85-94$ & 33 & 0.2 & 4 & 25.8 & 7.0 & 7.0 & 7.0 & 7.0 \\
\hline & total & 15,421 & 100.0 & 353 & & & & & \\
\hline
\end{tabular}

*1 Number of cases who lived more than 30 days.

*2 Number of cases who died within 30 days.

Mantel Chi-Square Test

\begin{tabular}{|c|c|c|c|c|c|c|c|c|c|c|c|}
\hline & PAT 1 & PAT 2 & PAT 3 & PAT 4 & PAT 5 & PAT 6 & PAT 7 & PAT 8 & PAT 9 & PAT 10 & PAT 11 \\
\hline \multirow{2}{*}{ PAT 1} & & & & & & $* *$ & $* * *$ & $* * *$ & $* * *$ & *** & **** \\
\hline & -1.00 & 6.01 & 0.94 & 1.31 & 1.34 & 7.66 & 84.46 & 259.63 & 374.12 & 286.94 & 36.45 \\
\hline \multirow{2}{*}{ PAT 2} & & & **** & $* * *$ & & & $* * *$ & *** & $* * *$ & $* * *$ & $* * *$ \\
\hline & 6.01 & -1.00 & 11.04 & 14.78 & 3.09 & 0.08 & 40.15 & 179.29 & 282.97 & 222.18 & 26.22 \\
\hline \multirow{2}{*}{ PAT 3} & & *** & & & & $* * *$ & *** & *** & $* * *$ & *** & $* * *$ \\
\hline & 0.94 & 11.04 & -1.00 & 0.00 & 4.92 & 14.19 & 94.88 & 260.94 & 369.53 & 296.06 & 45.76 \\
\hline \multirow{2}{*}{ PAT 4} & & *** & & & & *** & **** & *** & *** & $* * *$ & $* * *$ \\
\hline & 1.31 & 14.78 & 0.00 & -1.00 & 6.53 & 19.74 & 139.31 & 384.16 & 539.17 & 418.03 & 58.02 \\
\hline \multirow{2}{*}{ PAT 5} & & & & & & & $* * *$ & *** & $* * *$ & $* * *$ & $* * *$ \\
\hline & 1.34 & 3.09 & 4.92 & 6.53 & -1.00 & 3.69 & 107.96 & 376.44 & 568.96 & 455.38 & 58.60 \\
\hline \multirow{2}{*}{ PAT 6} & ** & & $* * *$ & $* * *$ & & & *** & *** & *** & *** & $* * *$ \\
\hline & 7.66 & 0.08 & 14.19 & 19.74 & 3.69 & -1.00 & 90.28 & 393.07 & 618.27 & 473.78 & 49.31 \\
\hline \multirow{2}{*}{ PAT 7} & *** & *** & *** & $* * *$ & $* * *$ & $* * *$ & & *** & *** & *** & $* * *$ \\
\hline & 84.46 & 40.15 & 94.88 & 139.31 & 107.96 & 90.28 & -1.00 & 114.55 & 279.65 & 237.63 & 19.95 \\
\hline \multirow{2}{*}{ PAT 8} & *** & *** & *** & *** & $* * *$ & $* * *$ & $* * *$ & & *** & *** & * \\
\hline & 259.63 & 179.29 & 260.94 & 384.16 & 376.44 & 393.07 & 114.55 & -1.00 & 54.24 & 78.04 & 6.20 \\
\hline \multirow{2}{*}{ PAT 9} & *** & *** & *** & $* * *$ & $* * *$ & $* * *$ & $* * *$ & $* * *$ & & *** & \\
\hline & 374.12 & 282.97 & 369.53 & 539.17 & 568.96 & 618.27 & 279.65 & 54.24 & -1.00 & 13.37 & 1.43 \\
\hline \multirow{2}{*}{ PAT10 } & *** & *** & *** & *** & *** & $* * *$ & $* * *$ & $* * *$ & *** & & \\
\hline & 286.94 & 222.18 & 296.06 & 418.03 & 455.38 & 473.78 & 237.63 & 78.04 & 13.37 & -1.00 & 0.08 \\
\hline \multirow{2}{*}{ PAT11 } & *** & *** & *** & *** & *** & *** & $* * *$ & * & & & \\
\hline & 36.45 & 26.22 & 45.76 & 58.02 & 58.60 & 49.31 & 19.95 & 6.20 & 1.43 & 0.08 & -1 \\
\hline
\end{tabular}


2) Glioblastoma (1984-2000) : Cumulative Survival Rate

\begin{tabular}{|c|c|c|c|c|c|c|c|c|c|}
\hline \multicolumn{2}{|c|}{ PATTERN } & \multicolumn{2}{|c|}{ NO. OF CASES $* 1$} & $* 2$ & 1-YEAR & 2-YEAR & 3 -YEAR & 4-YEAR & 5-YEAR \\
\hline & & & $\%$ & & $\%$ & $\%$ & $\%$ & $\%$ & $\%$ \\
\hline PAT 1 & $0-4$ & 33 & 0.6 & 6 & 32.3 & 25.5 & 21.9 & 21.9 & 21.9 \\
\hline PAT 2 & $5-9$ & 65 & 1.2 & 2 & 32.3 & 15.3 & 13.6 & 10.2 & 10.2 \\
\hline PAT 3 & $10-14$ & 67 & 1.2 & 2 & 59.1 & 27.6 & 21.1 & 16.2 & 12.8 \\
\hline PAT 4 & $15-24$ & 176 & 3.3 & 5 & 66.9 & 36.6 & 22.4 & 17.4 & 16.6 \\
\hline PAT 5 & $25-34$ & 271 & 5.0 & 2 & 70.2 & 37.4 & 27.0 & 21.3 & 16.1 \\
\hline PAT & $35-44$ & 499 & 9.3 & 13 & 72.1 & 38.2 & 24.0 & 20.1 & 15.9 \\
\hline PAT 7 & $45-54$ & 901 & 16.7 & 21 & 63.1 & 25.2 & 15.8 & 12.8 & 10.7 \\
\hline PAT $\varepsilon$ & $55-64$ & 1,476 & 27.4 & 38 & 53.8 & 18.5 & 10.0 & 6.8 & 4.7 \\
\hline PAT & $65-74$ & 1,413 & 26.3 & 52 & 43.1 & 12.4 & 5.9 & 4.1 & 2.4 \\
\hline PAT 10 & $75-84$ & 454 & 8.4 & 25 & 26.8 & 8.5 & 3.7 & 1.6 & 1.6 \\
\hline PAT 11 & $85-94$ & 25 & 0.5 & 2 & 20.8 & 3.0 & - & - & - \\
\hline & otal & 5,380 & 100.0 & 168 & & & & & \\
\hline
\end{tabular}

$* 1$ Number of cases who lived more than 30 days.

$* 2$ Number of cases who died within 30 days.

\section{Mantel Chi-Square Test}

\begin{tabular}{|c|c|c|c|c|c|c|c|c|c|c|c|}
\hline & PAT 1 & PAT 2 & PAT 3 & PAT 4 & PAT 5 & PAT 6 & PAT 7 & PAT 8 & PAT 9 & PAT 10 & PAT 11 \\
\hline PAT 1 & -1.00 & 0.37 & 0.01 & 0.69 & 1.11 & 1.60 & 0.22 & 0.23 & 1.30 & 2.29 & 0.66 \\
\hline PAT 2 & 0.37 & -1.00 & 1.04 & $\begin{array}{r}* * \\
4.55 \\
\end{array}$ & $\begin{array}{r}* * \\
6.64 \\
\end{array}$ & $\begin{array}{r}* * \\
9.05 \\
\end{array}$ & 3.44 & 0.40 & 0.10 & 1.13 & 0.36 \\
\hline PAT 3 & 0.01 & 1.04 & -1.00 & 0.54 & 0.95 & 1.28 & 0.01 & 1.86 & $\begin{array}{r}* * \\
5.05\end{array}$ & $\begin{array}{r}* * * \\
7.63 \\
\end{array}$ & 3.28 \\
\hline PAT 4 & 0.69 & $\begin{array}{r}* * \\
4.55 \\
\end{array}$ & 0.54 & -1.00 & 0.11 & 0.17 & 1.92 & $\begin{array}{r}* * * \\
10.71\end{array}$ & $\begin{array}{r}* * * * \\
20.59\end{array}$ & $\begin{array}{r}* * * * \\
24.97\end{array}$ & $\begin{array}{r}* * \\
7.21\end{array}$ \\
\hline PAT 5 & 1.11 & $\begin{array}{r}* * \\
6.64 \\
\end{array}$ & 0.95 & 0.11 & -1.00 & 0.01 & $\begin{array}{r}* \\
4.55 \\
\end{array}$ & $\begin{array}{r}* * * \\
19.49 \\
\end{array}$ & $\begin{array}{r}\text { **** } \\
35.22\end{array}$ & $\begin{array}{r}\text { **** } \\
40.45\end{array}$ & $\begin{array}{r}* * \\
9.42\end{array}$ \\
\hline PAT 6 & 1.60 & $\begin{array}{r}* * \\
9.05 \\
\end{array}$ & 1.28 & 0.17 & 0.01 & -1.00 & $\begin{array}{r}* * \\
7.72 \\
\end{array}$ & $\begin{array}{r}\text { *** } \\
32.67 \\
\end{array}$ & $\begin{array}{r}* * * \\
58.26\end{array}$ & $\begin{array}{r}\text { *** } \\
63.28 \\
\end{array}$ & $\begin{array}{r}\text { **** } \\
11.42\end{array}$ \\
\hline PAT 7 & 0.22 & 3.44 & 0.01 & 1.92 & $\begin{array}{r}* \\
4.55\end{array}$ & $\begin{array}{r}* * \\
7.72\end{array}$ & -1.00 & $\begin{array}{r}* * * \\
10.61\end{array}$ & $\begin{array}{r}\text { *** } \\
33.15\end{array}$ & $\begin{array}{r}\text { *** } \\
43.88\end{array}$ & $\begin{array}{r}* \\
6.04\end{array}$ \\
\hline PAT 8 & 0.23 & 0.40 & 1.86 & $\begin{array}{r}* * * \\
10.71 \\
\end{array}$ & $\begin{array}{r}* * * \\
19.49 \\
\end{array}$ & $\begin{array}{r}* * * \\
32.67 \\
\end{array}$ & $\begin{array}{r}* * * * \\
10.61 \\
\end{array}$ & -1.00 & $\begin{array}{r}* * \\
9.94 \\
\end{array}$ & $\begin{array}{r}* * * \\
23.25 \\
\end{array}$ & 3.06 \\
\hline PAT 9 & 1.30 & 0.10 & $\begin{array}{r}* \\
5.05 \\
\end{array}$ & $\begin{array}{r}* * * \\
20.59 \\
\end{array}$ & $\begin{array}{r}\text { *** } \\
35.22 \\
\end{array}$ & $\begin{array}{r}* * * \\
58.26 \\
\end{array}$ & $\begin{array}{r}* * * * \\
33.15 \\
\end{array}$ & $\begin{array}{r}* * * \\
9.94 \\
\end{array}$ & -1.00 & $\begin{array}{r}* * \\
6.46 \\
\end{array}$ & 1.17 \\
\hline PAT10 & 2.29 & 1.13 & $\begin{array}{r}* * \\
7.63 \\
\end{array}$ & $\begin{array}{r}* * * \\
24.97 \\
\end{array}$ & $\begin{array}{r}* * * \\
40.45 \\
\end{array}$ & $\begin{array}{r}* * * \\
63.28 \\
\end{array}$ & $\begin{array}{r}* * * \\
43.88 \\
\end{array}$ & $\begin{array}{r}* * * * \\
23.25 \\
\end{array}$ & $\begin{array}{r}* \\
6.46 \\
\end{array}$ & -1.00 & 0.11 \\
\hline PAT11 & 0.66 & 0.36 & 3.28 & $\begin{array}{r}* * \\
7.21\end{array}$ & $\begin{array}{r}* * * \\
9.42\end{array}$ & $\begin{array}{r}* * * \\
11.42\end{array}$ & $\begin{array}{r}* \\
6.04\end{array}$ & 3.06 & 1.17 & 0.11 & -1.00 \\
\hline
\end{tabular}


3) Astrocytoma (1984-2000) : Cumulative Survival Rate

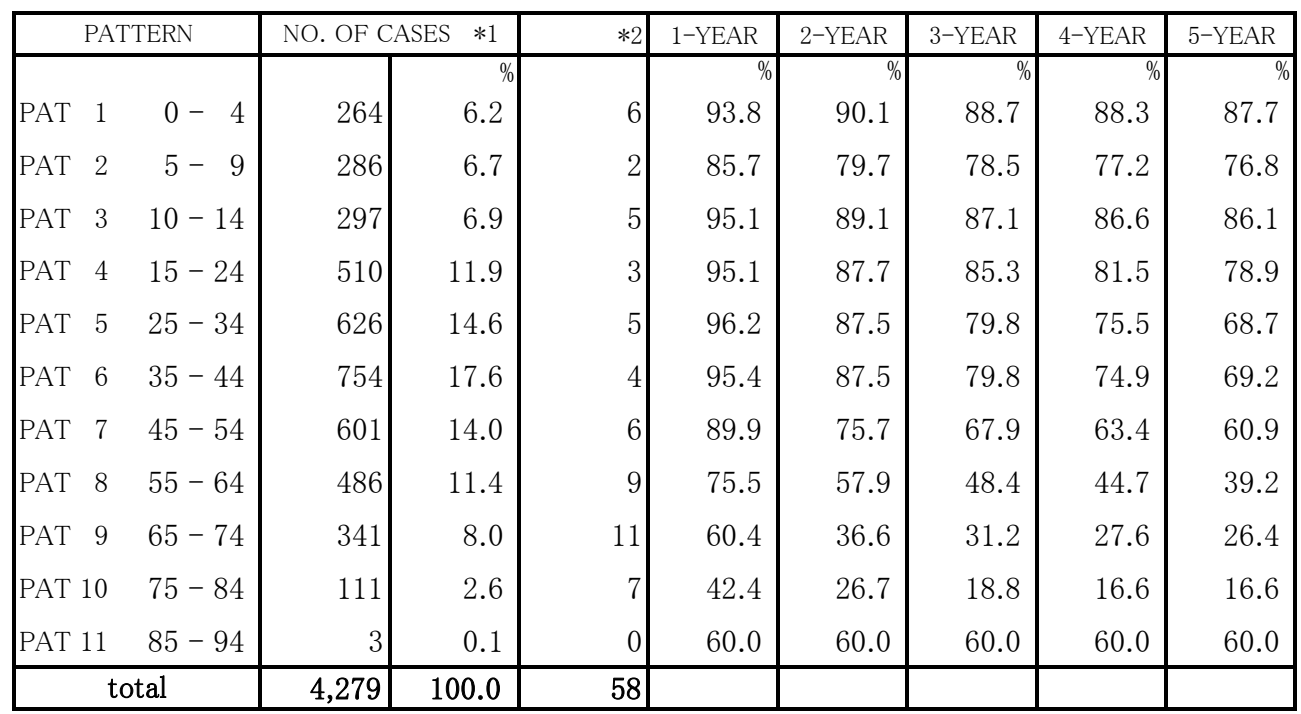

*1 Number of cases who lived more than 30 days.

$* 2$ Number of cases who died within 30 days.

Mantel Chi-Square Test

\begin{tabular}{|c|c|c|c|c|c|c|c|c|c|c|c|}
\hline & PAT 1 & PAT 2 & PAT 3 & PAT 4 & PAT 5 & PAT 6 & PAT 7 & PAT 8 & PAT 9 & PAT 10 & PAT 11 \\
\hline \multirow{2}{*}{ PAT 1} & & & & & *** & $* * *$ & $* * *$ & *** & $* * *$ & $* * *$ & \\
\hline & -1.00 & 9.29 & 0.19 & 5.46 & 19.72 & 20.11 & 40.59 & 100.68 & 133.96 & 106.79 & 1.99 \\
\hline \multirow{2}{*}{ PAT 2} & $* *$ & & ** & & & & *** & *** & $* * *$ & *** & \\
\hline & 9.29 & -1.00 & 7.53 & 1.43 & 1.04 & 1.06 & 11.05 & 56.08 & 85.12 & 63.20 & 0.43 \\
\hline \multirow{2}{*}{ PAT 3} & & & & & *** & *** & $* * *$ & $* * *$ & $* * *$ & $* * *$ & \\
\hline & 0.19 & 7.53 & -1.00 & 3.73 & 17.06 & 17.52 & 39.11 & 102.63 & 139.75 & 114.76 & 2.16 \\
\hline \multirow{2}{*}{ PAT 4} & & & & & ** & ** & *** & *** & *** & *** & \\
\hline & 5.46 & 1.43 & 3.73 & -1.00 & 7.38 & 7.74 & 31.34 & 115.44 & 171.53 & 142.49 & 1.34 \\
\hline \multirow{2}{*}{ PAT 5} & $* * *$ & & *** & ** & & & $* * *$ & *** & *** & *** & \\
\hline & 19.72 & 1.04 & 17.06 & 7.38 & -1.00 & 0.00 & 11.49 & 89.50 & 161.42 & 150.17 & 0.81 \\
\hline \multirow{2}{*}{ PAT 6} & $* * *$ & & *** & ** & & & $* * *$ & *** & $* * *$ & *** & \\
\hline & 20.11 & 1.06 & 17.52 & 7.74 & 0.00 & -1.00 & 12.77 & 99.66 & 178.19 & 157.06 & 0.75 \\
\hline \multirow{2}{*}{ PAT 7} & $* * *$ & *** & *** & $* * *$ & *** & $* * *$ & & *** & $* * *$ & *** & \\
\hline & 40.59 & 11.05 & 39.11 & 31.34 & 11.49 & 12.77 & -1.00 & 35.68 & 83.88 & 76.96 & 0.20 \\
\hline \multirow{2}{*}{ PAT 8} & $* * *$ & *** & *** & $* * *$ & *** & *** & *** & & $* * *$ & *** & \\
\hline & 100.68 & 56.08 & 102.63 & 115.44 & 89.50 & 99.66 & 35.68 & -1.00 & 12.82 & 19.75 & 0.04 \\
\hline \multirow{2}{*}{ PAT 9} & $* * *$ & $* * *$ & $* * *$ & $* * *$ & *** & $* * *$ & $* * *$ & *** & & & \\
\hline & 133.96 & 85.12 & 139.75 & 171.53 & 161.42 & 178.19 & 83.88 & 12.82 & -1.00 & 3.15 & 0.26 \\
\hline \multirow{2}{*}{ PAT 10} & $* * *$ & *** & *** & *** & *** & $* * *$ & *** & *** & & & \\
\hline & 106.79 & 63.20 & 114.76 & 142.49 & 150.17 & 157.06 & 76.96 & 19.75 & 3.15 & -1.00 & 0.58 \\
\hline PAT 11 & 1.99 & 0.43 & 2.16 & 1.34 & 0.81 & 0.75 & 0.20 & 0.04 & 0.26 & 0.58 & -1.00 \\
\hline
\end{tabular}


4) Anaplastic Astrocytoma( 1984-2000) : Cumulative Survival Rate

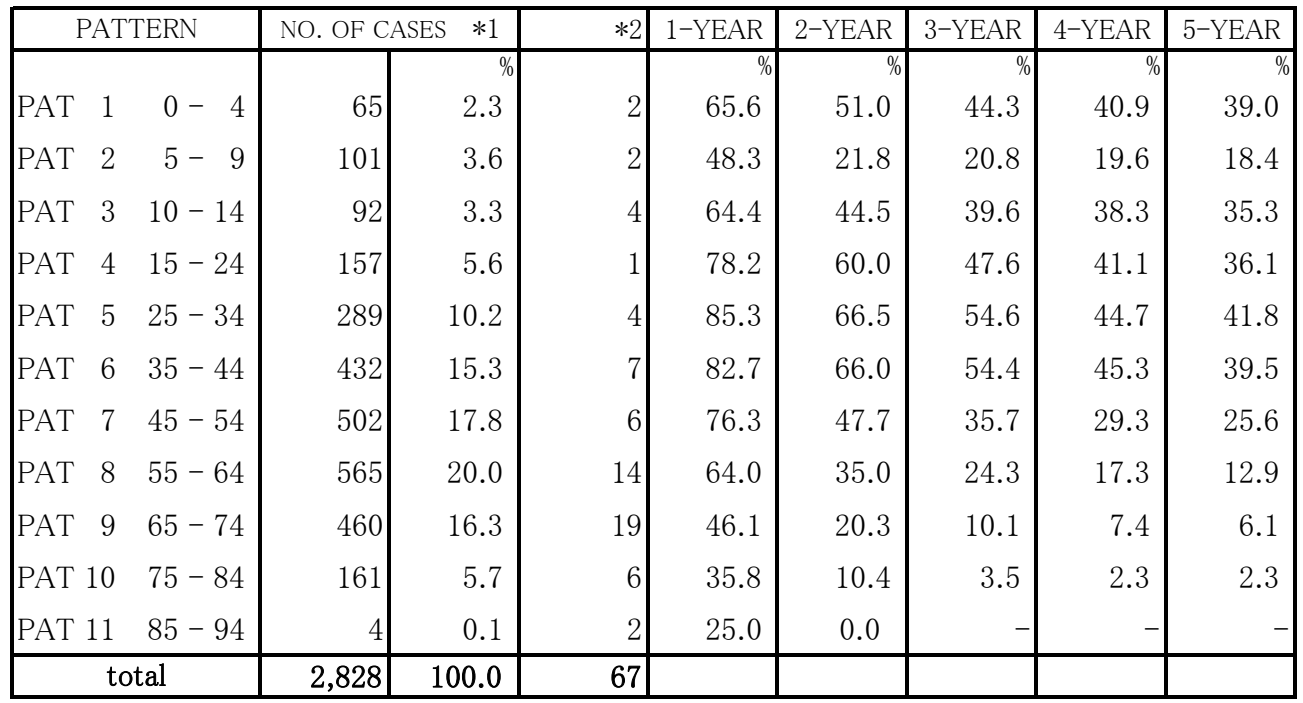

$* 1$ Number of cases who lived more than 30 days.

$* 2$ Number of cases who died within 30 days.

Mantel Chi-Square Test

\begin{tabular}{|c|c|c|c|c|c|c|c|c|c|c|c|}
\hline & PAT 1 & PAT 2 & PAT 3 & PAT 4 & PAT 5 & PAT 6 & PAT 7 & PAT 8 & PAT 9 & PAT 10 & PAT 11 \\
\hline PAT 1 & -1.00 & 3.82 & 0.11 & 0.05 & 1.32 & 0.78 & 0.84 & $\begin{array}{r}* * \\
7.25 \\
\end{array}$ & $\begin{array}{r}\text { **** } \\
15.73\end{array}$ & $\begin{array}{r}* * * \\
15.34\end{array}$ & 1.88 \\
\hline PAT 2 & 3.82 & -1.00 & 3.32 & $\begin{array}{r}* * * \\
10.57 \\
\end{array}$ & $\begin{array}{r}* * * * \\
25.38 \\
\end{array}$ & $\begin{array}{r}* * * \\
25.58\end{array}$ & $\begin{array}{r}* * \\
7.78 \\
\end{array}$ & 0.33 & 1.78 & 3.44 & 0.38 \\
\hline PAT 3 & 0.11 & 3.32 & -1.00 & 0.58 & 3.68 & 2.86 & 0.18 & $\begin{array}{r}* * \\
6.08 \\
\end{array}$ & $\begin{array}{r}* * * \\
15.75 \\
\end{array}$ & $\begin{array}{r}* * * \\
15.13 \\
\end{array}$ & 1.55 \\
\hline PAT 4 & 0.05 & $\begin{array}{r}\text { *** } \\
10.57\end{array}$ & 0.58 & -1.00 & 1.45 & 0.85 & 3.22 & $\begin{array}{r}* * * \\
18.45\end{array}$ & $\begin{array}{r}\text { **** } \\
39.01\end{array}$ & $\begin{array}{r}\text { *** } \\
37.81 \\
\end{array}$ & $\begin{array}{r}* \\
3.93 \\
\end{array}$ \\
\hline PAT 5 & 1.32 & $\begin{array}{r}* * * \\
25.38\end{array}$ & 3.68 & 1.45 & -1.00 & 0.18 & $\begin{array}{r}\text { **** } \\
15.11\end{array}$ & $\begin{array}{r}* * * \\
49.57\end{array}$ & $\begin{array}{r}\text { **** } \\
86.82\end{array}$ & $\begin{array}{r}* * * \\
74.40\end{array}$ & $6.62^{*}$ \\
\hline PAT 6 & 0.78 & $\begin{array}{r}* * * * \\
25.58 \\
\end{array}$ & 2.86 & 0.85 & 0.18 & -1.00 & $\begin{array}{r}* * * * \\
15.22 \\
\end{array}$ & $\begin{array}{r}* * * \\
56.90 \\
\end{array}$ & $\begin{array}{r}* * * \\
102.76 \\
\end{array}$ & $\begin{array}{r}* * * \\
81.32 \\
\end{array}$ & $\begin{array}{r}* \\
5.78 \\
\end{array}$ \\
\hline PAT 7 & 0.84 & $\begin{array}{r}* * \\
7.78 \\
\end{array}$ & 0.18 & 3.22 & $\begin{array}{r}* * * * \\
15.11 \\
\end{array}$ & $\begin{array}{r}* * * \\
15.22 \\
\end{array}$ & -1.00 & $\begin{array}{r}* * * * \\
14.52 \\
\end{array}$ & $\begin{array}{r}* * * \\
48.99 \\
\end{array}$ & $\begin{array}{r}* * * \\
44.26 \\
\end{array}$ & 2.95 \\
\hline PAT 8 & $\begin{array}{r}* * \\
7.25 \\
\end{array}$ & 0.33 & $\begin{array}{r}* \\
6.08 \\
\end{array}$ & $\begin{array}{r}* * * \\
18.45 \\
\end{array}$ & $\begin{array}{r}* * * \\
49.57 \\
\end{array}$ & $\begin{array}{r}* * * \\
56.90 \\
\end{array}$ & $\begin{array}{r}* * * \\
14.52 \\
\end{array}$ & -1.00 & $\begin{array}{r}* * * \\
13.96 \\
\end{array}$ & $\begin{array}{r}* * * \\
17.84 \\
\end{array}$ & 1.28 \\
\hline PAT 9 & $\begin{array}{r}* * * \\
15.73 \\
\end{array}$ & 1.78 & $\begin{array}{r}* * * * \\
15.75 \\
\end{array}$ & $\begin{array}{r}* * * \\
39.01 \\
\end{array}$ & $\begin{array}{r}* * * * \\
86.82 \\
\end{array}$ & $\begin{array}{r}* * * \\
102.76 \\
\end{array}$ & $\begin{array}{r}* * * \\
48.99 \\
\end{array}$ & $\begin{array}{r}* * * \\
13.96 \\
\end{array}$ & -1.00 & 2.10 & 0.33 \\
\hline PAT10 & $\begin{array}{r}* * * \\
15.34 \\
\end{array}$ & 3.44 & $\begin{array}{r}* * * \\
15.13 \\
\end{array}$ & $\begin{array}{r}* * * \\
37.81 \\
\end{array}$ & $\begin{array}{r}* * * \\
74.40 \\
\end{array}$ & $\begin{array}{r}* * * \\
81.32 \\
\end{array}$ & $\begin{array}{r}* * * \\
44.26 \\
\end{array}$ & $\begin{array}{r}* * * \\
17.84\end{array}$ & 2.10 & -1.00 & 0.08 \\
\hline PAT11 & 1.88 & 0.38 & 1.55 & $\begin{array}{r}* * \\
3.93\end{array}$ & $\begin{array}{r}* \\
6.62\end{array}$ & $\begin{array}{r}* \\
5.78\end{array}$ & 2.95 & 1.28 & 0.33 & 0.08 & -1.00 \\
\hline
\end{tabular}




\section{Result of Treatment for Metastatic Brain Tumor}

1) Mode of Metastasis (1984-2000): Cumulative Survival Rate

\begin{tabular}{|l|r|r|r|r|r|r|r|r|}
\hline \multicolumn{1}{|c|}{ PATTERN } & \multicolumn{2}{|c|}{ NO. OF CASES $* 1$} & $* 2$ & 1-YEAR & 2-YEAR & 3-YEAR & 4-YEAR & 5-YEAR \\
\hline PAT 1 single & & $\%$ & & $\%$ & $\%$ & $\%$ & $\%$ & $\%$ \\
PAT 2 multiple & 7,019 & 60.2 & 335 & 47.6 & 29.1 & 21.7 & 18.3 & 16.2 \\
PAT 3 menig. ca. & 4,357 & 37.4 & 292 & 33.5 & 16.9 & 10.5 & 8.2 & 6.4 \\
\hline \multicolumn{1}{|c|}{ total } & 274 & 2.4 & 78 & 21.9 & 8.1 & 5.8 & 5.8 & 4.6 \\
\hline
\end{tabular}

*1 Number of cases who lived more than 30 days.

*2 Number of cases who died within 30 days.

Mantel Chi-Square Test :

Significancy between PAT1 and PAT2 ( $<<0.001)$, PAT1 and PAT3 $(p<0.001)$

2) Clinical Grading (1984-2000): Cumulative Survival Rate

\begin{tabular}{|c|c|c|c|c|c|c|c|c|c|}
\hline & PATTERN & NO. OF CA & S $* 1$ & $* 2$ & $1-\mathrm{YEAR}$ & $2-\mathrm{YEAR}$ & 3 -YEAR & 4-YEAR & 5 -YEAR \\
\hline & & & $\%$ & & $\%$ & $\%$ & $\%$ & $\%$ & $\%$ \\
\hline PAT 1 & no symptoms & 523 & 4.5 & 17 & 59.7 & 41.4 & 34.2 & 27.1 & 24.2 \\
\hline PAT 2 & complaints only & 1,166 & 10.1 & 37 & 55.7 & 37.2 & 28.2 & 24.9 & 21.9 \\
\hline PAT 3 & focal signs & 6,920 & 59.7 & 282 & 42.5 & 23.9 & 16.6 & 13.6 & 11.6 \\
\hline PAT 4 & increased ICP & 2,112 & 18.2 & 144 & 34.1 & 18.1 & 12.5 & 10.4 & 9.0 \\
\hline PAT 5 & $\begin{array}{l}\text { disturbance of } \\
\text { consciousness }\end{array}$ & 744 & 6.4 & 145 & 25.9 & 13.3 & 9.3 & 8.0 & 8.0 \\
\hline PAT 6 & coma & 104 & 0.9 & 70 & 20.8 & 10.0 & 8.0 & 8.0 & 6.0 \\
\hline PAT 7 & $\begin{array}{l}\text { respiratory } \\
\text { impairment }\end{array}$ & 13 & 0.1 & 9 & 7.7 & 0.0 & - & - & - \\
\hline & total & 11,582 & 100.0 & 704 & & & & & \\
\hline
\end{tabular}

$* 1$ Number of cases who lived more than 30 days.

$* 2$ Number of cases who died within 30 days.

\section{Mantel Chi-Square Test}

\begin{tabular}{|c|c|c|c|c|c|c|c|}
\hline & AT 1 & PAT 2 & PAT 3 & PAT 4 & PAT 5 & PAT 6 & PAT 7 \\
\hline PAT 1 & -1.00 & 0.98 & $\begin{array}{r}\text { **** } \\
24.76\end{array}$ & $\begin{array}{r}\text { **** } \\
36.04\end{array}$ & $\begin{array}{r}\text { *** } \\
36.55\end{array}$ & $\begin{array}{r}* * * \\
15.53\end{array}$ & 4.98 \\
\hline PAT 2 & 0.98 & -1.00 & $\begin{array}{r}* * * \\
29.66 \\
\end{array}$ & $\begin{array}{r}* * * \\
44.82 \\
\end{array}$ & $\begin{array}{r}* * * \\
41.83 \\
\end{array}$ & $\begin{array}{r}\text { **** } \\
13.25\end{array}$ & 4.01 \\
\hline PAT 3 & $\begin{array}{r}* * * * \\
24.76\end{array}$ & $\begin{array}{r}* * * \\
29.66\end{array}$ & -1.00 & $\begin{array}{r}* * \\
9.86 \\
\end{array}$ & $\begin{array}{r}* * * \\
13.86\end{array}$ & $\begin{array}{r}* \\
3.87\end{array}$ & 1.71 \\
\hline PAT 4 & $\begin{array}{r}* * * * \\
36.04 \\
\end{array}$ & $\begin{array}{r}* * * \\
44.82 \\
\end{array}$ & $\begin{array}{r}* * \\
9.86 \\
\end{array}$ & -1.00 & 2.04 & 1.15 & 0.91 \\
\hline PAT 5 & $\begin{array}{r}* * * * \\
36.55 \\
\end{array}$ & $\begin{array}{r}* * * \\
41.83 \\
\end{array}$ & $\begin{array}{r}* * * * \\
13.86 \\
\end{array}$ & 2.04 & -1.00 & 0.18 & 0.44 \\
\hline PAT 6 & $\begin{array}{r}* * * * \\
15.53 \\
\end{array}$ & $\begin{array}{r}* * * * \\
13.25\end{array}$ & $\begin{array}{r}* * \\
3.87\end{array}$ & 1.15 & 0.18 & -1.00 & 0.21 \\
\hline PAT 7 & $\begin{array}{r}* \\
4.98 \\
\end{array}$ & $\begin{array}{r}* \\
4.01\end{array}$ & 1.71 & 0.91 & 0.44 & 0.21 & -1.00 \\
\hline
\end{tabular}


3) Primary Site and Survival Rate (1984-2000): Cumulative Survival Rate

\begin{tabular}{|ll|r|r|r|r|r|r|r|r|}
\hline & PATTERN & \multicolumn{2}{|c|}{ NO. OF CASES $* 1$} & $* 2$ & 1-YEAR & 2-YEAR & 3-YEAR & 4-YEAR & 5-YEAR \\
\hline PAT 1 lung & & $\%$ & & $\%$ & $\%$ & $\%$ & $\%$ & $\%$ \\
PAT 2 & breast & 5,656 & 60.4 & 283 & 40.7 & 21.1 & 14.1 & 11.2 & 9.8 \\
PAT 3 & 1,007 & 10.8 & 55 & 50.2 & 31.1 & 21.2 & 17.4 & 13.8 \\
PAT 4 kidno-intestine & 1,552 & 16.6 & 133 & 31.7 & 16.2 & 11.5 & 10.0 & 8.7 \\
PAT 5 & 588 & 6.3 & 21 & 49.9 & 31.0 & 23.7 & 21.5 & 18.0 \\
PAT 6 head and neck & 193 & 2.1 & 12 & 42.3 & 30.9 & 24.1 & 23.1 & 23.1 \\
\hline \multicolumn{1}{|c|}{ total } & 365 & 3.9 & 18 & 54.6 & 40.5 & 30.8 & 26.6 & 23.5 \\
\hline
\end{tabular}

*1 Number of cases who lived more than 30 days.

$* 2$ Number of cases who died within 30 days.

\section{Mantel Chi-Square Test}

\begin{tabular}{|c|c|c|c|c|c|c|}
\hline & PAT 1 & PAT 2 & PAT 3 & PAT 4 & PAT 5 & PAT 6 \\
\hline \multirow{2}{*}{ PAT 1} & & $* * *$ & $*$ & $* *$ & & $* * *$ \\
\hline & -1.00 & 10.66 & 5.94 & 9.85 & 3.70 & 18.84 \\
\hline \multirow{2}{*}{ PAT 2} & $* * *$ & & *** & & & \\
\hline & 10.66 & -1.00 & 18.24 & 0.31 & 0.21 & 4.46 \\
\hline \multirow{2}{*}{ PAT 3} & & *** & & $* * *$ & $*$ & $* * *$ \\
\hline & 5.94 & 18.24 & -1.00 & 15.43 & 6.17 & 22.81 \\
\hline \multirow{2}{*}{ PAT 4} & ** & & $* * *$ & & & \\
\hline & 9.85 & 0.31 & 15.43 & -1.00 & 0.00 & 1.90 \\
\hline PAT 5 & 3.70 & 0.21 & $\begin{array}{r}* \\
6.17\end{array}$ & 0.00 & -1.00 & 0.74 \\
\hline \multirow{2}{*}{ PAT 6} & $* * *$ & & *** & & & \\
\hline & 18.84 & 4.46 & 22.81 & 1.90 & 0.74 & -1.00 \\
\hline
\end{tabular}

4) Radiation Therapy

a) Metastatic Brain Tumor from Lung Cancer (1984-2000): Cumulative Survival Rate

\begin{tabular}{|c|c|c|c|c|c|c|c|c|}
\hline PATTERN & \multicolumn{2}{|c|}{ NO. OF CASES $* 1$} & $* 2$ & 1-YEAR & 2-YEAR & 3-YEAR & 4-YEAR & 5-YEAR \\
\hline & & 9 & & 9 & $\%$ & $\%$ & $\%$ & $\%$ \\
\hline PAT 0 & 2,132 & 38.5 & 215 & 34.5 & 18.8 & 13.6 & 12.1 & 11.6 \\
\hline irradiated & 3,411 & 61.5 & 63 & 44.4 & 22.5 & 14.6 & 10.9 & 8.9 \\
\hline total & 5,543 & 100.0 & 278 & & & & & \\
\hline
\end{tabular}

*1 Number of cases who lived more than 30 days.

*2 Number of cases who died within 30 days.

Mantel Chi-Square Test : No Significancy Detected between Two of PATs

b) Metastatic Brain Tumor from Breast Cancer (1984-2000): Cumulative Survival Rate

\begin{tabular}{|ll|r|r|r|r|r|r|r|r|}
\hline & PATTERN & \multicolumn{2}{|c|}{ NO. OF CASES $* 1$} & $* 2$ & 1-YEAR & 2-YEAR & 3-YEAR & 4-YEAR & 5-YEAR \\
\hline \multirow{2}{*}{ PAT 0 none } & 361 & 36.4 & 41 & 48.6 & 32.7 & 22.0 & 17.8 & 15.6 \\
PAT 1 irradiated & 630 & 63.6 & 14 & 51.3 & 29.9 & 20.3 & 16.9 & 12.4 \\
\hline \multicolumn{2}{|c|}{ total } & 991 & 100.0 & 55 & & & & & \\
\hline
\end{tabular}

*1 Number of cases who lived more than 30 days.

$* 2$ Number of cases who died within 30 days. 
5) Extent of Cancer and Survival Rate

a) Recurrence of Cancer: Primary Site

i) Lung Cancer (1984-2000): Cumulative Survival Rate

\begin{tabular}{|c|c|c|c|c|c|c|c|c|}
\hline PATTERN & NO. OF C & $* 1$ & $* 2$ & 1-YEAR & 2-YEAR & 3-YEAR & 4-YEAR & 5-YEAR \\
\hline & & $\%$ & & & & $\%$ & $\%$ & $\%$ \\
\hline $\operatorname{Rec}(-)$ & 2,127 & 50.4 & 68 & 50.0 & 29.2 & 20.6 & 16.5 & 14.4 \\
\hline PAT $1 \quad \operatorname{Rec}(+)$ & 2,090 & 49.6 & 140 & 32.5 & 14.4 & 8.3 & 6.4 & 5.5 \\
\hline total & 4,217 & 100.0 & 208 & & & & & \\
\hline
\end{tabular}

*1 Number of cases who lived more than 30 days.

$* 2$ Number of cases who died within 30 days.

Mantel Chi-Square Test : Significant by $p<0.001$

ii ) Breast Cancer (1984-2000) : Cumulative Survival Rate

\begin{tabular}{|r|r|r|r|r|r|r|r|r|}
\hline PATTERN & \multicolumn{2}{|c|}{ NO. OF CASES $* 1$} & $* 2$ & 1-YEAR & 2-YEAR & 3-YEAR & 4-YEAR & 5-YEAR \\
\hline PAT 0 Rec $(-)$ & 653 & 75.5 & 30 & 54.8 & 35.1 & 24.3 & 20.2 & 15.5 \\
PAT 1 Rec $(+)$ & 212 & 24.5 & 16 & 33.3 & 18.4 & 12.1 & 8.5 & 6.1 \\
\hline total & 865 & 100.0 & 46 & & & & & \\
\hline
\end{tabular}

*1 Number of cases who lived more than 30 days.

*2 Number of cases who died within 30 days.

Mantel Chi-Square Test : Significant by $p<0.01$

b) Metastasis of Other Organ and Survival Rate

i) Lung Cancer (1984-2000): Cumulative Survival Rate

\begin{tabular}{|c|r|r|r|r|r|r|r|r|}
\hline PATTERN & \multicolumn{2}{|c|}{ NO. OF CASES $* 1$} & $* 2$ & 1-YEAR & 2-YEAR & 3-YEAR & 4-YEAR & 5-YEAR \\
\hline PAT 0 meta $(-)$ & 3,158 & 71.8 & 105 & 48.1 & 26.7 & 18.0 & 14.2 & 12.4 \\
PAT 1 meta (+) & 1,239 & 28.2 & 86 & 26.2 & 9.7 & 5.6 & 4.8 & 3.6 \\
\hline total & 4,397 & 100.0 & 191 & & & & & \\
\hline
\end{tabular}

$* 1$ Number of cases who lived more than 30 days.

$* 2$ Number of cases who died within 30 days.

Mantel Chi-Square Test : Significant by $p<0.001$

ii ) Breast Cancer (1984-2000): Cumulative Survival Rate

\begin{tabular}{|c|r|r|r|r|r|r|r|r|}
\hline PATTERN & \multicolumn{2}{|c|}{ NO. OF CASES $* 1$} & $* 2$ & 1-YEAR & 2-YEAR & 3-YEAR & 4-YEAR & 5-YEAR \\
\hline PAT 0 meta $(-)$ & 376 & 41.4 & 11 & 62.1 & 42.7 & 29.6 & 24.4 & 20.0 \\
PAT 1 meta $(+)$ & 533 & 58.6 & 35 & 42.4 & 22.8 & 15.3 & 12.7 & 9.0 \\
\hline total & 909 & 100.0 & 46 & & & & & \\
\hline
\end{tabular}

*1 Number of cases who lived more than 30 days.

$* 2$ Number of cases who died within 30 days.

Mantel Chi-Square Test : Significant by $p<0.001$ 
6) Results of Treatment for Metastatic Brain Tumor

a) Lung Cancer

i) Operation (1984-2000) : Cumulative Survival Rate

\begin{tabular}{|ll|r|r|r|r|r|r|r|r|}
\hline & PATTERN & \multicolumn{2}{|c|}{ NO. OF CASES $* 1$} & $* 2$ & 1-YEAR & 2-YEAR & 3-YEAR & 4-YEAR & 5-YEAR \\
\hline PAT 0 & none & 1,503 & 27.0 & 130 & 31.2 & 14.8 & 10.0 & 7.0 & 5.1 \\
PAT 1 & decompression & 151 & 2.7 & 20 & 22.7 & 7.6 & 3.8 & 2.5 & 1.3 \\
PAT 2 & biopsy or partial & 439 & 7.9 & 25 & 31.7 & 12.3 & 7.2 & 5.8 & 4.6 \\
PAT 3 & $50 \%$ resected & 162 & 2.9 & 9 & 37.6 & 13.8 & 5.5 & 1.1 & - \\
PAT 4 & $75 \%$ resected & 404 & 7.3 & 21 & 36.3 & 16.7 & 8.2 & 6.5 & 6.5 \\
PAT 5 & $95 \%$ resected & 956 & 17.2 & 20 & 45.1 & 23.2 & 14.6 & 12.0 & 8.7 \\
PAT 6 & total removal & 1,947 & 35.0 & 54 & 50.2 & 29.1 & 21.1 & 17.4 & 16.3 \\
\hline & total & 5,562 & 100.0 & 279 & & & & & \\
\hline
\end{tabular}

$* 1$ Number of cases who lived more than 30 days.

*2 Number of cases who died within 30 days.

Manatel Chi-Square Test

\begin{tabular}{|c|c|c|c|c|c|c|c|}
\hline & $\begin{array}{l}\text { PAT } 0 \\
\end{array}$ & $\begin{array}{l}\text { PAT } 1 \\
\end{array}$ & $\begin{array}{l}\text { PAT } 2 \\
\end{array}$ & $\begin{array}{l}\text { PAT } 3 \\
\end{array}$ & PAT 4 & $\begin{array}{l}\text { PAT } 5 \\
\end{array}$ & $\begin{array}{l}\text { PAT } 6 \\
\end{array}$ \\
\hline PAT 0 & -100 & 135 & 005 & 0.00 & 0.37 & $\begin{array}{r}* * \\
928\end{array}$ & $\begin{array}{r}* * * \\
3586\end{array}$ \\
\hline PAT 1 & 1.35 & -1.00 & 0.93 & 0.96 & 2.21 & $\begin{array}{r}* * * \\
7.38\end{array}$ & $\begin{array}{r}* * * \\
14.23\end{array}$ \\
\hline PAT 2 & 0.05 & 0.93 & -1.00 & 0.04 & 0.53 & $\begin{array}{r}* * \\
6.24\end{array}$ & $\begin{array}{r}* * * \\
18.67\end{array}$ \\
\hline PAT 3 & 0.00 & 0.96 & 0.04 & -1.00 & 0.09 & 2.39 & $\begin{array}{r}* \\
6.57 \\
\end{array}$ \\
\hline PAT 4 & 0.37 & 2.21 & 0.53 & 0.09 & -1.00 & 2.54 & $\begin{array}{r}* * * \\
10.79 \\
\end{array}$ \\
\hline PAT 5 & $\begin{array}{r}* * \\
9.28 \\
\end{array}$ & $\begin{array}{r}\text { ** } \\
7.38 \\
\end{array}$ & $\begin{array}{r}* \\
6.24 \\
\end{array}$ & 2.39 & 2.54 & -1.00 & $\begin{array}{r}* \\
5.49 \\
\end{array}$ \\
\hline PAT 6 & $\begin{array}{r}* * * \\
35.86\end{array}$ & $\begin{array}{r}* * * \\
14.23\end{array}$ & $\begin{array}{r}* * * \\
18.67\end{array}$ & $\begin{array}{r}* \\
6.57\end{array}$ & $\begin{array}{r}* * * \\
10.79\end{array}$ & $\begin{array}{r}* * \\
5.49\end{array}$ & -1.00 \\
\hline
\end{tabular}

ii ) Clinical Grading (1984-2000): Cumulative Survival Rate

\begin{tabular}{|c|c|c|c|c|c|c|c|c|c|}
\hline & PATTERN & NO. OF C & $E S \quad * 1$ & $* 2$ & 1-YEAR & 2-YEAR & 3-YEAR & 4-YEAR & 5-YEAR \\
\hline & & & $\%$ & & $\%$ & $\%$ & $\%$ & $\%$ & $\%$ \\
\hline PAT 1 & no symptoms & 324 & 5.8 & 13 & 53.7 & 35.9 & 27.6 & 20.7 & 17.5 \\
\hline PAT 2 & complaints only & 513 & 9.2 & 16 & 51.1 & 31.3 & 22.3 & 18.3 & 15.6 \\
\hline PAT 3 & focal signs & 3,463 & 61.9 & 139 & 41.5 & 20.6 & 13.1 & 10.3 & 8.7 \\
\hline PAT 4 & increased ICP & 934 & 16.7 & 46 & 35.3 & 16.9 & 11.1 & 9.1 & 8.4 \\
\hline PAT 5 & $\begin{array}{l}\text { disturbance of } \\
\text { consciousness }\end{array}$ & 323 & 5.8 & 47 & 22.3 & 9.4 & 7.8 & 7.8 & 7.8 \\
\hline PAT 6 & coma & 31 & 0.6 & 16 & 18.0 & 3.6 & 3.6 & 3.6 & 3.6 \\
\hline PAT 7 & $\begin{array}{l}\text { respiratory } \\
\text { impairment }\end{array}$ & 6 & 0.1 & 3 & 0.0 & - & - & - & - \\
\hline & total & 5,594 & 100.0 & 280 & & & & & \\
\hline
\end{tabular}

*1 Number of cases who lived more than 30 days.

$* 2$ Number of cases who died within 30 days. 
Mantel Chi-Square Test

\begin{tabular}{|c|c|c|c|c|c|c|c|}
\hline & PAT 1 & PAT 2 & PAT 3 & PAT 4 & PAT 5 & PAT 6 & PAT 7 \\
\hline \multirow{2}{*}{ PAT 1} & & & & $* * *$ & $* * *$ & $*$ & \\
\hline & -1.00 & 0.43 & 9.40 & 11.48 & 13.64 & 4.60 & 1.79 \\
\hline \multirow{2}{*}{ PAT 2} & & & $* *$ & ** & $* * *$ & & \\
\hline & 0.43 & -1.00 & 7.39 & 10.02 & 13.34 & 3.78 & 1.56 \\
\hline \multirow{2}{*}{ PAT 3} & & ** & & & ** & & \\
\hline & 9.40 & 7.39 & -1.00 & 1.86 & 6.93 & 1.56 & 0.88 \\
\hline \multirow{2}{*}{ PAT 4} & $* * *$ & & & & & & \\
\hline & 11.48 & 10.02 & 1.86 & -1.00 & 2.13 & 0.81 & 0.57 \\
\hline \multirow{2}{*}{ PAT 5} & *** & $* * *$ & ** & & & & \\
\hline & 13.64 & 13.34 & 6.93 & 2.13 & -1.00 & 0.09 & 0.19 \\
\hline PAT 6 & $\begin{array}{r}* \\
4.60 \\
\end{array}$ & 3.78 & 1.56 & 0.81 & 0.09 & -1.00 & 0.10 \\
\hline PAT 7 & 1.79 & 1.56 & 0.88 & 0.57 & 0.19 & 0.10 & -1.00 \\
\hline
\end{tabular}

iii ) Effects of Various Treatment (1984-2000) : Cumulative Survival Rate

\begin{tabular}{|ll|r|r|r|r|r|r|r|r|}
\hline & PATTERN & \multicolumn{2}{|c|}{ NO. OF CASES $* 1$} & $* 2$ & 1-TEAR & 2-YEAR & 3-YEAR & 4-YEAR & 5-YEAR \\
\hline PAT 1 & ope + rad. & 216 & 16.2 & 8 & 45.7 & 28.0 & 17.4 & 14.2 & 14.2 \\
PAT 2 & ope. alone & 820 & 61.4 & 32 & 55.8 & 33.1 & 24.8 & 20.2 & 17.9 \\
PAT 3 rad. alone & 299 & 22.4 & 10 & 35.5 & 17.9 & 9.9 & 5.4 & 4.0 \\
\hline \multicolumn{1}{|c|}{ total } & 1,335 & 100.0 & 50 & & & & & \\
\hline
\end{tabular}

$* 1$ Number of cases who lived more than 30 days.

$* 2$ Number of cases who died within 30 days.

Mantel Chi-Square Test : Significancy Detected Only between PAT2 and PAT3 by $p<0.001$

b) Breast Cancer

i) Operation (1984-2000): Cumulative Survival Rate

\begin{tabular}{|ll|r|r|r|r|r|r|r|r|}
\hline & PATTERN & \multicolumn{2}{|c|}{ NO. OF CASES $* 1$} & $* 2$ & 1-YEAR & 2-YEAR & 3-YEAR & 4-YEAR & 5-YEAR \\
\hline PAT 0 & none & 248 & 25.0 & 31 & 41.9 & 20.8 & 12.3 & 10.6 & 8.7 \\
PAT 1 & decompression & 38 & 3.8 & 7 & 23.5 & 10.1 & 10.1 & 10.1 & 10.1 \\
PAT 2 & biopsy or partial & 68 & 6.9 & 1 & 48.8 & 34.3 & 19.6 & 16.8 & 10.7 \\
PAT 3 & $50 \%$ resected & 27 & 2.7 & 1 & 57.7 & 27.8 & 13.9 & 2.8 & - \\
PAT 4 & 75\% resected & 77 & 7.8 & 4 & 40.1 & 23.1 & 13.9 & 13.9 & 0.0 \\
PAT 5 & 95\% resected & 199 & 20.1 & 4 & 50.5 & 32.0 & 22.7 & 16.5 & 16.5 \\
PAT 6 & total removal & 335 & 33.8 & 6 & 61.4 & 41.9 & 30.9 & 26.7 & 20.3 \\
\hline \multicolumn{2}{c}{ total } & 992 & 100.0 & 54 & & & & & \\
\hline
\end{tabular}

*1 Number of cases who lived more than 30 days.

$* 2$ Number of cases who died within 30 days. 
Mantel Chi-Square Test

\begin{tabular}{|c|c|c|c|c|c|c|c|}
\hline & PAT 0 & PAT 1 & PAT 2 & PAT 3 & PAT 4 & PAT 5 & PAT 6 \\
\hline PAT 0 & -1.00 & 0.66 & 0.59 & 0.04 & 0.00 & 2.11 & $\begin{array}{r}* * * \\
10.42\end{array}$ \\
\hline PAT 1 & 0.66 & -1.00 & 1.20 & 0.94 & 0.37 & 2.08 & 5.53 \\
\hline PAT 2 & 0.59 & 1.20 & -1.00 & 0.19 & 0.53 & 0.08 & 1.60 \\
\hline PAT 3 & 0.04 & 0.94 & 0.19 & -1.00 & 0.12 & 0.27 & 1.93 \\
\hline PAT 4 & 0.00 & 0.37 & 0.53 & 0.12 & -1.00 & 1.10 & 4.67 \\
\hline PAT 5 & 2.11 & 2.08 & 0.08 & 0.27 & 1.10 & -1.00 & 2.10 \\
\hline PAT 6 & $\begin{array}{r}* * * \\
10.42\end{array}$ & $\begin{array}{r}* * \\
5.53\end{array}$ & 1.60 & 1.93 & $\begin{array}{r}* \\
4.67\end{array}$ & 2.10 & -1.00 \\
\hline
\end{tabular}

ii ) Clinical Grading (1984-2000): Cumulative Survival Rate

\begin{tabular}{|c|c|c|c|c|c|c|c|c|}
\hline PATTERN & NO. OF C & $\mathrm{ES} * 1$ & $* 2$ & 1-YEAR & 2-YEAR & 3-YEAR & 4-YEAR & 5-YEAR \\
\hline & & & & $\%$ & $\%$ & $\%$ & $\%$ & $\%$ \\
\hline PAT 1 no symptoms & 32 & 3.2 & 1 & 83.1 & 66.0 & 55.0 & 48.1 & 40.1 \\
\hline PAT 2 complaints only & 134 & 13.5 & 3 & 68.3 & 51.7 & 38.1 & 34.4 & 29.8 \\
\hline PAT 3 focal signs & 515 & 52.0 & 9 & 52.1 & 31.2 & 19.8 & 15.8 & 11.9 \\
\hline PAT 4 increased ICP & 252 & 25.4 & 25 & 37.3 & 17.8 & 12.6 & 9.9 & 7.9 \\
\hline PAT $5 \begin{array}{l}\text { disturbance of } \\
\text { consciousness }\end{array}$ & 53 & 5.3 & 13 & 33.3 & 26.2 & 17.9 & 11.4 & 11.4 \\
\hline PAT 6 coma & 4 & 0.4 & 4 & 0.0 & - & - & - & - \\
\hline PAT $7 \begin{array}{l}\text { respiratory } \\
\text { impairment }\end{array}$ & 1 & 0.1 & 0.0 & 0.0 & - & - & - & - \\
\hline total & 991 & 100.0 & 55 & & & & & \\
\hline
\end{tabular}

*1 Number of cases who lived more than 30 days.

$* 2$ Number of cases who died within 30 days.

Mantel Chi-Square Test

\begin{tabular}{|c|c|c|c|c|c|c|c|}
\hline & PAT 1 & PAT 2 & PAT 3 & PAT 4 & PAT 5 & PAT 6 & PAT 7 \\
\hline PAT 1 & -1.00 & 1.23 & $\begin{array}{r}* * \\
7.24\end{array}$ & $\begin{array}{r}* * * \\
10.28\end{array}$ & $\begin{array}{r}* \\
6.06\end{array}$ & $\begin{array}{r}* * \\
4.79\end{array}$ & 1.69 \\
\hline PAT 2 & 1.23 & -1.00 & $\begin{array}{r}* * \\
8.56 \\
\end{array}$ & $\begin{array}{r}* * * * \\
15.20 \\
\end{array}$ & $\begin{array}{r}* \\
5.63 \\
\end{array}$ & 2.70 & 0.72 \\
\hline PAT 3 & $\begin{array}{r}* * \\
7.24 \\
\end{array}$ & $\begin{array}{r}* * \\
8.56 \\
\end{array}$ & -1.00 & $\begin{array}{r}* * \\
4.12 \\
\end{array}$ & 0.62 & 1.12 & 0.28 \\
\hline PAT 4 & $\begin{array}{r}* * * \\
10.28 \\
\end{array}$ & $\begin{array}{r}* * * \\
15.20 \\
\end{array}$ & $\begin{array}{r}* * \\
4.12 \\
\end{array}$ & -1.00 & 0.06 & 0.43 & 0.11 \\
\hline PAT 5 & $\begin{array}{r}* \\
6.06 \\
\end{array}$ & $\begin{array}{r}* \\
5.63 \\
\end{array}$ & 0.62 & 0.06 & -1.00 & 0.30 & 0.08 \\
\hline PAT 6 & $\begin{array}{r}* \\
4.79 \\
\end{array}$ & 2.70 & 1.12 & 0.43 & 0.30 & -1.00 & 0.00 \\
\hline PAT 7 & 1.69 & 0.72 & 0.28 & 0.11 & 0.08 & 0.00 & -1.00 \\
\hline
\end{tabular}


iii) Effects of Various Treatment (1984-2000) : Cumulative Survival Rate

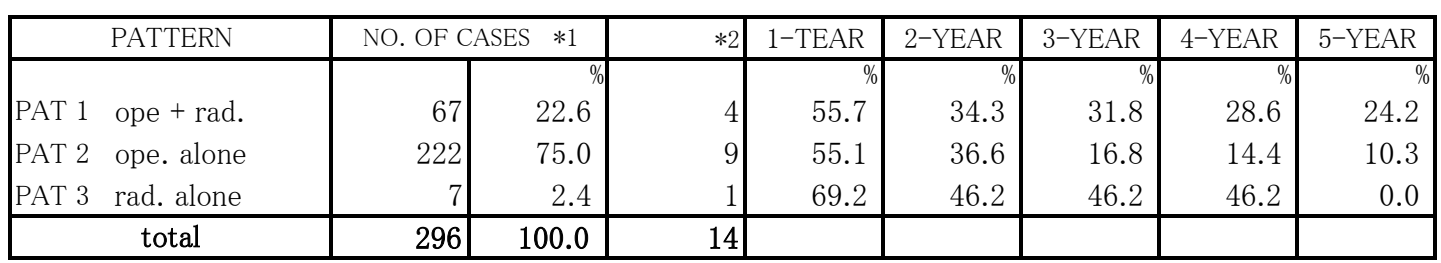

*1 Number of cases who lived more than 30 days.

$* 2$ Number of cases who died within 30 days.

Mantel Chi-Square Test : No significancy Detected between Each of PATs

c) Gastric Cancer

i) Operation (1984-2000): Cumulative Survival Rate

\begin{tabular}{|c|c|c|c|c|c|c|c|c|c|}
\hline & PATTERN & NO. OF C & $\mathrm{ES} \quad * 1$ & $* 2$ & 1-YEAR & 2-YEAR & 3-YEAR & 4-YEAR & 5-YEAR \\
\hline & & & $\%$ & & $\%$ & $\%$ & $\%$ & $\%$ & $\%$ \\
\hline PAT 0 & none & 140 & 30.0 & 30 & 22.2 & 10.1 & 10.1 & 7.6 & 7.6 \\
\hline PAT 1 & decompression & 17 & 3.6 & 10 & 0.0 & - & - & - & - \\
\hline PAT 2 & biopsy or partial & 22 & 4.7 & 8 & 26.8 & 6.7 & 0.0 & - & - \\
\hline PAT 3 & $50 \%$ resected & 11 & 2.4 & 1 & 18.2 & 9.1 & 0.0 & - & - \\
\hline PAT 4 & $75 \%$ resected & 32 & 6.9 & 4 & 34.5 & 29.2 & 17.5 & 17.5 & 5.8 \\
\hline PAT 5 & $95 \%$ resected & 71 & 15.2 & 3 & 42.0 & 11.3 & 8.4 & 8.4 & 8.4 \\
\hline PAT 6 & total removal & 174 & 37.3 & 13 & 39.2 & 25.1 & 21.1 & 17.6 & 17.6 \\
\hline & total & 467 & 100.0 & 69 & & & & & \\
\hline
\end{tabular}

$* 1$ Number of cases who lived more than 30 days.

*2 Number of cases who died within 30 days.

Mantel Chi-Square Test : No Significancy Detected between Each Two of PATs

\section{ii ) Clinical Grading (1984-2000): Cumulative Survival Rate}

\begin{tabular}{|c|c|c|c|c|c|c|c|c|c|}
\hline & PATTERN & NO. OF & ES $* 1$ & $* 2$ & 1-YEAR & 2-YEAR & 3-YEAR & 4-YEAR & 5-YEAR \\
\hline & & & $\%$ & & $\%$ & $\%$ & $\%$ & $\%$ & $\%$ \\
\hline PAT 1 & no symptoms & 7 & 1.5 & 0 & 60.0 & 0.0 & - & - & - \\
\hline PAT 2 & complaints only & 42 & 8.9 & 2 & 40.5 & 16.7 & 16.7 & 16.7 & 0.0 \\
\hline PAT 3 & focal signs & 293 & 62.2 & 24 & 34.2 & 19.4 & 13.9 & 11.5 & 11.5 \\
\hline PAT 4 & increased ICP & 92 & 19.5 & 19 & 19.8 & 7.2 & 7.2 & 7.2 & 2.4 \\
\hline PAT 5 & $\begin{array}{l}\text { disturbance of } \\
\text { consciousness }\end{array}$ & 31 & 6.6 & 15 & 20.0 & 12.0 & 12.0 & 12.0 & 12.0 \\
\hline PAT 6 & coma & 6 & 1.3 & 11 & 20.0 & - & - & - & - \\
\hline PAT 7 & $\begin{array}{l}\text { respiratory } \\
\text { impairment }\end{array}$ & 0 & 0.0 & 0 & - & - & - & - & - \\
\hline & total & 471 & 100.0 & 71 & & & & & \\
\hline
\end{tabular}

*1 Number of cases who lived more than 30 days.

$* 2$ Number of cases who died within 30 days. 
iii) Effects of Various Treatment (1984-2000) : Cumulative Survival Rate

\begin{tabular}{|ll|r|r|r|r|r|r|r|r|}
\hline & PATTERN & \multicolumn{2}{|c|}{ NO. OF CASES $* 1$} & $* 2$ & 1-TEAR & 2-YEAR & 3-YEAR & 4-YEAR & 5-YEAR \\
\hline PAT 1 & ope + rad. & 5 & 2.7 & 1 & 11.1 & $\%$ & $\%$ & $\%$ & $\%$ \\
PAT 2 & ope. alone & 179 & 96.2 & 20 & 38.8 & 21.1 & 15.8 & 14.2 & - \\
PAT 3 & rad. alone & 2 & 1.1 & 0 & 33.3 & - & - & - & - \\
\hline \multicolumn{1}{c|}{ total } & 186 & 100.0 & 21 & & & & & - \\
\hline
\end{tabular}

$* 1$ Number of cases who lived more than 30 days.

$* 2$ Number of cases who died within 30 days.

Mantel Chi-Square Test : No Significancy Detected Between Each Two of PATs 